

\section{DISCLAIMER}

This report was prepared as an account of work sponsored by an agency of the United States Government. Neither the United States Government nor any agency Thereof, nor any of their employees, makes any warranty, express or implied, or assumes any legal liability or responsibility for the accuracy, completeness, or usefulness of any information, apparatus, product, or process disclosed, or represents that its use would not infringe privately owned rights. Reference herein to any specific commercial product, process, or service by trade name, trademark, manufacturer, or otherwise does not necessarily constitute or imply its endorsement, recommendation, or favoring by the United States Government or any agency thereof. The views and opinions of authors expressed herein do not necessarily state or reflect those of the United States Government or any agency thereof. 


\section{DISCLAIMER}

Portions of this document may be illegible in electronic image products. Images are produced from the best available original document. 


\title{
PRELIMINARY MARKET ANALYSIS FOR CUSTOMER SIDE OF THE METER THERMAL-ENERGY STORAGE
}

\author{
ENERGY STORAGE SYSTEMS \\ OFFICE OF CONSERVATION AND SOLAR ENERGY \\ DEPARTMENT OF ENERGY
}

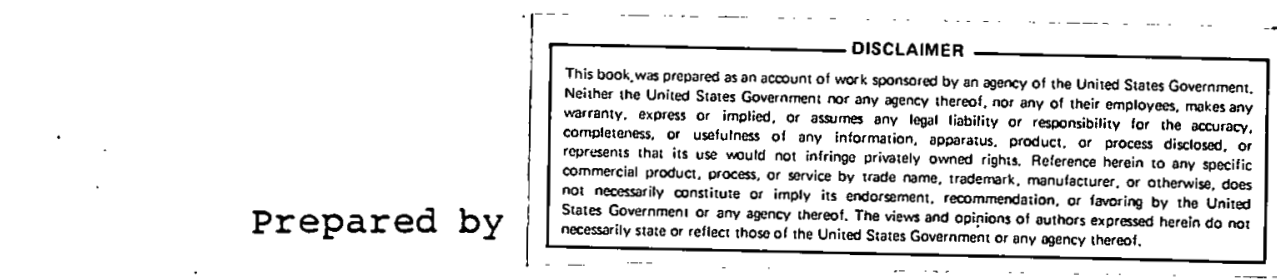

Booz, Allen \& Hamilton Inc.

Energy and Environment Division

4330 East-West Highway

Bethesda, Maryland 20014

C. I. Rust, officer-in-Charge

W. F. Adolfson, Project Manager

R. M. Grant

P. A. Weinberger

J. D. White

D. M. Eriedman

December 31, 1979

CONTRACT NO. EM-78-C-01-5114

TASK NO. 16600-009-007 


\section{THIS PAGE}

\section{WAS INTENTIONALLY LEFT BLANK}


I N D E X O F E X H I B I T S

Page

Number

I-1 Projected Maximum TES Potential 8

I-2 DOE and NERC Energy Regions

I-3 Maximum Cool storage Available by NERC Regions

I-4 Maximum Heat Storage Available by NERC Regions

I-5 Example of General Service Rate

II-1 Economic Competitiveness of TES and Conventional Systems in the Residential sector

II-2 Commercial Energy Use by Building Type

II-3 Economic Competitiveness of TES and Conventional systems in the commercial sector

II-4 TES Market by Fuel Type

IT-5 New Space Heating Demand in Single and Multifamily Housing

II-6 New water Heating Demand in Single and Multifamily Housing

II-7 New Air Conditioning Demand in Single and Multifamily Housing

II-8 New Air Conditioning Demand in Commercial offices, Schools and Retail stores

II-9 New Water Heating Demand in Commercial Offices, Schools and Retail stores 
II-I0 TES Market by End-Use 35

III-1 Projected TES Market by the Year $2000 \quad 37$

III-2 Manufacturers Surveyed/Industry Status 45

III-3 Annual Market for Residential and
Commercial TES

III-4 Cumulative Demand for Residential and
Commercial TES

III-5 Cumulative Demand by End-Use: United
States

- III-6 Cumulative Demand by End-Use: DOE Region 53

A-1 Regional Rate Differential Requirements for a 4-Year Payback: Residential TES Systems

A-2 National Rate Differential Requirements for a 4-Year Payback: Commercial TES Systems

A-3 Life Cycle Costing Formula Used for Calculating Residential System Costs

A-4 Commercial Life Cycle Costing Formula

B-1 Common Assumptions for Residential Space Heating Life Cycle Cost Analysis

B-2 Regionally Specific Assumptions for Residential Space Heating

B-3 Iife Cycle Cost Analysis for Residential Space Heating

B-4 Common Assumptions for Residential Water Heating Life Cycle Cost Analysis

B-5 Reglonally Specific Assumptions for Residential Water Heating

B-6 Life Cycle Cost Analysis for Residential water Heating 


$$
\begin{aligned}
& \text { B-7 Common Assumptions for Residential Air } \\
& \text { Conditioning Life Cycle Cost Analysis } \\
& \text { B-8 Regionally Specific Assumptions for } \\
& \text { Residential Air Conditioning } \\
& \text { B-9 Life Cycle Cost Analysis for Residential } \\
& \text { Air Conditioning } \\
& \text { B-IO Life Cycle Cost Analysis for office } \\
& \text { Buildings } \\
& \text { B-ll Life Cycle Cost Analysis for Educational } \\
& \text { Buildings } \\
& \text { B-12 Life Cycle Cost Analysis for Retail } \\
& \text { Stores } \\
& \text { C-I New Space Heating Units Installed in } \\
& \text { Single and Multifamily Housing } \\
& \text { C-2 New Water Heating Units Installed in } \\
& \text { Single and Multifamily Housing } \\
& \text { c-3 New Air Conditioning Units Installed } \\
& \text { in single and Multifamily Housing } \\
& \text { D-1 Unit Demand Estimates }
\end{aligned}
$$


PRELIMINARY MARKET ANALYSIS FOR CUSTOMER SIDE OF THE METER THERMAL ENERGY STORAGE

Customer side of the meter thermal energy storage (TES) systems identified for early commercialization by the U.S. Department of Energy (DOE) are storage space heating, storage water heating, and storage air conditioning. Utilization of TES technology could benefit the electric utilities and their customers as well as provide significant national benefits. By installing TES to match a portion of their electric energy needs at traditional peak periods, consumers can shift their pattern of electrical demand (load) so that the same total energy can be distributed more evenly throughout the day. The reduced variation between on-peak and off-peak demand should improve the utility's load factor with concomitant cost savings resulting from greater use of larger, more efficient baseload generating units and reduced consumption

- of premium fuels for peak power generation. In addition, the reduced growth of peak demand should defer the need for capital expenditures for construction of new capacity. The benefit to the TES customer would be a possible reduction of annual energy costs.

Over the past 30 years the European experience with similar TES technology has demonstrated that these potential benefits are realizable. Electric storage heating systems have been successfully commercialized in England and West Germany and, to a lesser degree, in France, Switzerland, Austria, Belgium and Ireland. Offered in England as a low-cost method of space heating, TES systems were commercialized using time-of-day electric energy rates as part of a load management contract in which the utility controlled the operation of the system. Today, the British and West Germans each have approximately 20 GW of installed storage capacity, which has led to a more balanced electrical load for utilities.l

Although TES hot water and space heating systems have been used extensively in Europe, the U.S. market has been slow to develop. One of the many factors affecting the commercialization of TES in the U.S. is the recognition of a domestic market. The literature has not quantified the market well, although indications are that a market exists. The purpose of this report is to examine the potential market size tor TES space heating, water hoating and air conditioning in the residential and commercial sectors. 
The overall conclusion of this report is that a significant market for TES could develop over the next 20 years, but the market depends on many complicating factors. The analysis to estimate the size of the TES market considers:

- The load variations for electric utilities

- The comparative economics of TES and available alternatives

- The requirements for new installations

- Market penetration

- Manufacturing capability.

Based on these and other intrinsic factors, the TES market is described in terms of the electric utility perspective, the end-user perspective and the expected market:

Based on balancing the on- and off-peak loads, by the year 2000, nearly $130 \mathrm{GW}$ of TES capacity appears to be beneficial for electric utilities, although the utilization of TES is highly utility specific.

- Large parts of the residential and commercial markets having high usage of electrical and oil-burning units could be economically attractive for TES from the end-users' perspective.

- The TES market for space heating, water heating, and air conditioning could burgeon into a multigigawatt opportunity with the development of Eavorable utility rate designs and an aggressive public awareness program.

These measures that describe the TES market are discussed briefly in the following paragraphs.

The amount of TES capacity that could benefit electric utilities is about 14 percent of the annual system peak load. This potential was estimated from the effect of TES on balancing the seasonal and daily load variations of representative electric utility systems. Based on current National Electric Reliability Council (NERC) projections of load growth, the potential TES market, on a national basis, from the perspective of the electric utilities is as follows: 


\begin{tabular}{l} 
- $\quad 62 \mathrm{GW}$ by 1980 \\
- $\quad 96 \mathrm{GW}$ by 1985 \\
- $\quad 113 \mathrm{GW}$ by 1990 \\
- $\quad 130 \mathrm{GW}$ by 1995 \\
\hline
\end{tabular}

The practical application of TES on electric utility systems, however, is highly utility specific. These estimates are especially sensitive to:

Seasonal and daily load characteristics. The TES technology likely to be selected by electric utilities and the enthusiasm for TES was inferred from considering the size of the regional summer and winter peak loads projected by NERC:

- The 65 percent of the utilitiy industry that is summer peaking will encourage their customers to install storage cooling.

- The 35 percent of the industry that is winter peaking will encourage their customers to install storage space heating.

- Since water heating peaks can coincide with space heating peaks most winterpeaking utilities can benefit from storage water heating.

- Generation mix. The specific mix of base, intermediate and peak load generating capacity must be such that there is a sufficient economic base load capacity available to charge the TES systems and that TES are economically competitive with alternative options for meeting peak loads.

- Utility rate designs. The availability of rate designs that allow the customer to take advantage. of reduced electricity costs during off-peak periods is a necessary condition for the widespread utilization of TES. Even declining block. rate designs. Will encourage some commercial customers to use TES, but will provide little incentive for residential customers. Some form of timeof-use or time-of-day rates are required to provide most commercial and residential customers with the incentive to install TES. In some cases, establishing the rates needed to make TES economical may require new rate designs. 
Electric utility system characteristics place an upper bound on the amount of capacity that can be shifted from on-peak to off-peak. These results were used in determining the expected market.

The potential TES market can also be addressed from the perspective of the end user. The parts of the market that are attractive for TES were identified on the basis of economics and the demographics of new installations. The economics of TES and conventional oil, gas and electric alternatives are compared regionally on a life cycle cost basis. The economic analysis assumes the existence of a rate design that will offer the customer a rate differential between on-peak and off-peak electricity prices sufficient to achieve a 4-year payback. In some cases, this may require a change from the current rate design:

- Residential TES space heating rate differentials must range from 3.3 to $9.3 \mathrm{k} / \mathrm{Kwh}$

- Residential TES water heating requires about a $26 /$ Kwh differential

- Residential TES air conditioning rates require a minimum differential of $11.4 \mathrm{c} / \mathrm{KWh}$

- Commercial TES space heating and water heating rate differentials must not exceed $2 \mathrm{c} / \mathrm{KWh}$.

While the economic feasibility of TES is highly situation-specific, TES is generally an attractive option in large parts of the residential and commercial markets having high usage of electric and oil-burning units. Given the above rate differentials:

- Geographically, TES space heating is applicable to those markets served by winter peaking utilities; TES air conditioning, by summer peaking utilities; and TES water heating, throughout the U.S.

- TES could displace over 90 percent of the electric system and nearly 70 percent of the oil-burning systems for strictiy economic reasons.

- TES is economically competitive with over 90 percent of conventional air conditioning, principally commercial, and over 65 percent of residential space heating. 
However, decisions are never solely economic. Despite the economic advantage of TES compared to a few heat pumps and gas-burning units, the private sector perceives that TES will not displace these systems significantly. Factors regarding customer acceptance and behavior are considered in the estimates of the expected market.

The expected market for TES is likely to burgeon. into a multi-gigantic opportunity over the next 20 years. While it is currently very small, the TES market should increase as utility rate designs favorable to TES are established and public awareness increases. An estimate of the expected market depends on many complicating factors - both supply and demand related, and the analysis included informed judgements regarding market penetration. The private sector expects that the TES market will constitute about 5 percent of the new installations with which TES is economical. Since TES could be economically attractive on a life cycle cost basis with over 70 percent of conventional space heating, water heating and air conditioning systems, the resultant market for TES could account for 4 percent of the annual residential and commercial demand for these applications. In particular:

- Residential storage space heating and commercial air conditioning should dominate the TES market., consisting of about 80 percent of the TES demand

- The remaining 20 percent is comprised of residential water heating and air conditioning and commercial water heating

- Generally, the development of a market in commercial space heating is not expected to be large.

Manufacturers have indicated that they will expand production as the TES market develops. Generally, TES equipment is being marketed by HVAC companies as an extension of their current business, although for several firms TES is their only product. Anticipated plans to expand production appear to be sufficient to meet a moderately growing market in the early years. However, if the TES market were underestimated, European manufacturers would be available to supplement production of space heating units. 
Based on these considerations and the many factors intrinsic to them, the cumulative market size could be about $168 \mathrm{GW}$ of installed TES capacity in the next 20 years :

- TES air conditioning comprises $121 \mathrm{GW}$

- TES space heating comprises $39 \mathrm{GW}$

- TES water heating comprises $8 \mathrm{GW}$

These values are consistent with the amount of TES capacity that could benefit electric utilities, because the space heating and air conditioning demands are assumed to be mutually exclusive.

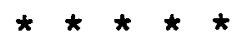

These findings are discussed in more detail in the three chapters of this report. 
I. POTENTIAL MARKET FROM ELECTRIC UTILITIES PERSPECTIVE

Distributed TES systems are one of several energy management techniques being considered by electric utilities to improve system load characteristics. Both summer and winter peaking utilities perceive a benefit from the utilization of TES. In the words of one utility official: "Since electric thermal storage equipment helps us to operate more efficiently, it also helps us to control both our capital and operating costs. For the consumer, this means sharing in these benefits in the form of lower electric bills."

A considerable amount of TES capacity appears to be beneficial to electric utilities, based on utility system load characteristics. An indication of the magnitude of this potential can be obtained from a recent EPRI Study ${ }^{2}$ which estimated that up to 14 percent of the annual system peak load could be served by daily cycling energy storage systems for a typical electric utility system. This estimate was derived from a statistical analysis of representative U.S. electric utility systems' load characteristics and the capability of TES to balance the on-peak and offpeak electrical loads. On a national scale--based on projected load growth--this is nearly $130 \mathrm{GW}$ by the year 2000, as shown in Exhibit I-I. These estimates are adapted from annual summer and winter peak load projections of the National Electric Reliability Council (NERC), and represent current assumptions concerning fuel availability, price increases and consumer behavior.

The regional potential for TES is projected from the size of the summer and winter peaks within the region. Since electric utilities have the capability to share load within power pools, which reach across DOE regions, peak load characteristics for DOE regions are inferred from NERC regional loads. Exhibit I-2 indicates the overlap among DOE and NERC regions.

The practical application of TES systems within regions is highly utility specific. The TES market from electric utilities' perspective is especially 


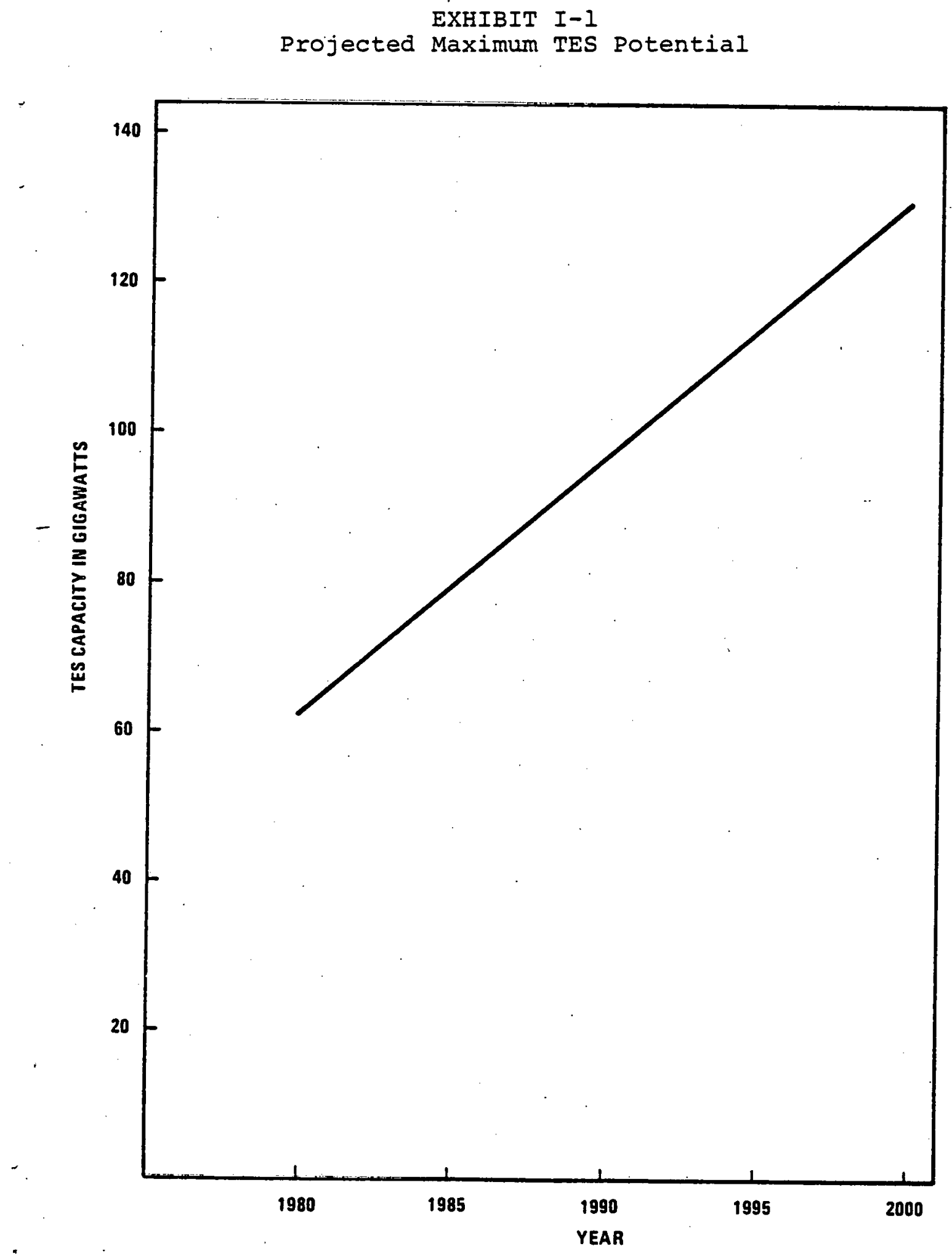

8 


\section{DOE AND NERC ENERGY REGIONS}

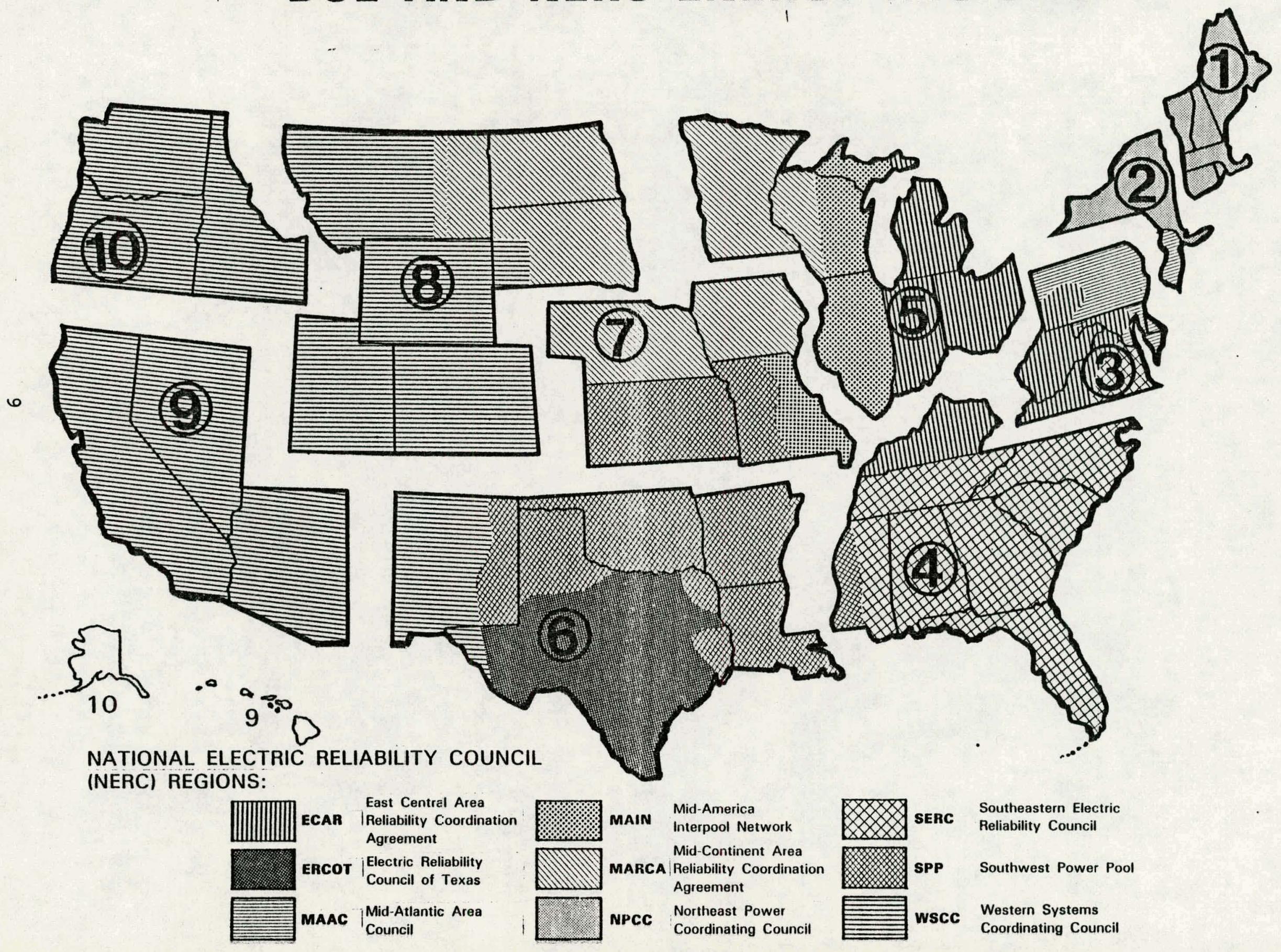


sensitive to the following factors:

- Seasonal and daily load characteristics

- Generation mix

- Utility rates structure

The following sections discuss the effect of these factors on the potential utilization of TES from electric utilities' perspective.

1. SEASONAI AND DAILY LOAD CHARACTERISTICS DETERMINE THE TYPE OF TES TECHNOLOGY AND THE DEGREE TO WHICH TES MIGHT BE ENCOURAGED

The form of TES selected and the vigor with which individual utilities will promote the technology will be determined by three criteria:

- Season in which the utility reaches peak load

- The difference between the daily peak and the lowest daily demand

- The daily seasonal and annual system load factors.

In addition, in determining the actual kilowatt goals for TES, the utilities will make allowances for scheduled and unscheduled generation outages.

There are three forms of TES currently attractive to the utility industry:

- Space heating

- Air conditioning

- Domestic hot water.

In the following sections, each of these will be discussed in relation to the utility type which is most likely to adopt the technology as a load management method.

I) The 65 Percent of the Utility Industry Which Is Summer Peaking Will Direct Their TES Marketing Efforts Towards Storage Cooling

Based on current information obtained from NERC, seven of the nine regions are currently summer peaking. Exhibit I-3 depicts the 10-year projected 
EXHIBIT I-3

Maximum Cool storage (MW) Available

By NERC Regions

\begin{tabular}{l|r|r|r}
\multicolumn{2}{c|}{1978} & 1983 & 1988 \\
\hline ECAR & 8,682 & 10,830 & 13,401 \\
ERCOT & 5,919 & 5,150 & 6,597 \\
MAAC & 4,454 & 5,329 & $.6,161$ \\
MAIN & 4,641 & 6,009 & 7,392 \\
MARCA & 2,501 & 5,383 & 4,329 \\
NPCC & 4,883 & 15,364 & 6,813 \\
SERC & 11,414 & 7,331 & 19,548 \\
SPP & 5,487 & 12,561 & 9,782 \\
WSCC & 9,461 & 71,799 & 8,411 \\
TOTAL & 55,442 & &
\end{tabular}


theoretical maximum megawatts available for TES cooling by NERC region. The average growth in available megawatts for TES cooling is 4.9 percent per year. These numbers indicate that there is substantial market for the stored cooling technology and a reasonable annual growth to sustain the TES-cooling industry.

Summer peaking utilities have a greater daily load variation than winter peaking systems, due to the high variability in air conditioning loads. To limit the growth of the generation peak or to allow growth in the base load, summer peaking utilities have a tremendous incentive to use TES.

There are two possible conditions which may cause a utility to be summer peaking:

- The service territory encompasses large commercial urban areas, e.g., New York City

- The service territories are located in hot and humid climates.

Utilities encompassing large commercial urban areas within their service territories are usually summer peaking. This situation is due to the current trend in commercial building design which requires a substantial amount of electric air conditioning. The commercial air conditioning load follows closely the sun's path across the sky and is determined by the occupancy use and the building's thermal design and operation. As these requirements generally occur between the hours of $10 \mathrm{a} . \mathrm{m}$. and 4 p.m., a large summer peak is experienced by utilities in these circumstances. On the other hand, the residential class will contribute relatively little to the summer peak. The individual residential air conditioning loads are extremely small, 3 to $5 \mathrm{~kW}$ per house, compared to commercial loads, but during the last decade, the new and retrofit residential air conditioning market accelerated to the point where there is some peak contribution. However, this rapid growth will not have a major influence on the peak because of the non-coincidence of individual air conditioning demands within the class.

Therefore, the greatest opportunity for TES will be to help check the air conditioning growth in the commercial class, since this load places the greatest burden on generation capacity. 
Utilities located in hot and humid climatic regions with only a small commercial cooling load will also tend to be a summer peaking utility. In these utilities, the peak is directly created by a combination of the residential and commercial classes' cooling. Therefore, utilities in this situation will have to limit the growth on both classes or use TES to allow growth in base load capacity.

(2) Winter Peaking Utilities Which Account for 35 Percent of All Utilities Will Encourage Their Customers to Install storage space Heating.

From the information supplied by NERC, only two of the nine regions are presently winter peaking and one additional region projected to become winter peaking over the next ten years. Exhibit I-4 depicts the 10-year projected theoretical maximum megawatts available for TES heating by NERC region. The average available megawatts growth through 1988 is expected to be 4.9 percent per year. As seen with the cooling technology the market appears to be sound and with good growth potential.

Winter peaking utilities are characterized by their large residential class space heating loads and, to a much lesser extent, the commercial class load. The commercial class has a small space heating load compared to the residential class since there are large heat gains attributable to people, lights, and equipment, causing commercial structures to require a substantial amount of cooling during the heating season.

The winter daily peak usually occurs between 6 p.m. and $10 \mathrm{p} . \mathrm{m}$. because the peak is being controlled by the residential heating demand. This is caused by the residential customer's use of electric stoves, washers, dryers, lights, and entertainment devices which combine with the increased demand for electric heat due to the evening outdoor temperature drop. This indicates the off-peak time includes both the late night period, after midnight, and also the early morning period from 6 a.m. to mid afternoon, 4 p.m. Therefore, residential customers have reasonable flexibility in terms of their off-peak charging times. (charging periods in utilities that also experience morning peaks are somewhat more restrictivel. 


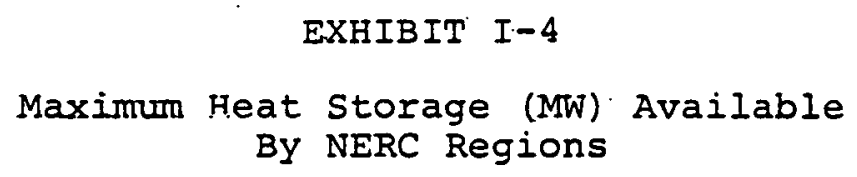

\begin{tabular}{l|r|r|r} 
& 1978 & 1983 & 1988 \\
\hline ECAR & 8,793 & 11,153 & 14,040 \\
MAAC & 2,908 & 3,732 & 4,791 \\
MAIN. & 4,012 & 4,839 & 5,720 \\
MARCA & 3,928 & 5,009 & 6,356 \\
NPCC. & 2,300 & 3,099 & 4,023 \\
SERC & 4,797 & 5,861 & 6,837 \\
SPP & 11,400 & 15,842 & 20,117 \\
WSCC. & 3,969 & 5,285 & 7,030 \\
& 9,702 & 12,193 & 14,918 \\
TOTAL & & & \\
& & &
\end{tabular}


Domestic hot water heating will also impact the winter peak, as discussed in the next section.

(3) Most Winter Peaking Utilities Can Benefit From Storage Domestic Hot Water Heating

During discussions with various utilities around the country it was evident that storage domestic hot water is a technology familiar to the utility industry. For years, utilities have promoted the concept of off-peak water heating using a combination time clock/kWh meter to perform this task. The winter peaking utilities have been the leaders in this effort since water heating peaks can coincide with the space

heating peaks. Therefore, the winter peaking companies will continue to promote this technology.

2. THE SPECIFIC MIX OF BASE LOAD, INTERMEDIATE, AND PEAK LOAD GENERATING CAPACITY--AS WELL AS THE USE OF LARGESCALE ENERGY STORAGE--EFFECTS THE RELATIVE ECONOMICS OF TES

The applicability of TES to a specific electric utility system is strongly dependent on the types of generating facilities, load duration curves for each generating unit, and the associated costs of generation. In particular, for TES to be attractive:

- There must be sufficient economic base load capacity available to charge the TES systems

- Distributed TES systems must be economically competitive with alternative forms of peak generation.

TES systems will not be attractive for those electric utilities that have:

\footnotetext{
- Excess capacity/high reserve margins

- Hydro-pumped storage facilities

- Baseload hydro generation

- Relatively little dependence on oil- and gas- . fired generation.
}

The application of TES to appropriate utility systems can result in more effective utilization of existing generating capacity, and in the long run, defer new capacity additions by:

Lowering reserve requirements/increasing reserve margins 
- More effective utilization of baseload generating facilities, in the form of:

- Increased capacity factor

- Improved plant heat rate

- Reducing dependence on costly fossil fuels normally used for peak generation.

3. ALTHOUGH TRADITIONAL BLOCK RATE STRUCTURES WILI ENCOURAGE SOME COMMERCIAL CUSTOMERS, TIME OF USE/TIME OF DAY RATES WILL PROVIDE INCENTIVES FOR BOTH COMMERCIAL AND RESIDENTIAL CUSTOLIERS

The market potential for TES depends largely on the availability of rate designs which allow TES system owners to utilize electric energy at off-peak times when it is least expensive.

Traditional Declining Block Rate Structures Will Encourage Some Commercial Customers to Use TES

The traditional declining block rate structure which has been the standard for many years in the utility industry can actually encourage certain commercial customers to install some form of TES. This encouragement occurs in the general service rates since the rates are composed of a demand and an energy component.

The demand component of the general service rate is usually a declining block rate which means that each additional unit of demand used will cost less than its predecessor. Some utilities' demand rates are ratcheted, that is, the highest demand reached in any month will be the demand the customer is billed for the next eleven months; unless a higher demand is achieved. Therefore, commercial customers who have a ratcheted demand rate with extreme changes in kilowatt monthly demands will be willing to look at methods to reduce their peak. As an example, if the peak is caused by air conditioning equipment during. the summer months, storage cooling will become attractive.

The energy component of the general service rate is also a declining block rate which would appear to have no value in promoting TES. However, the rate at which a commercial customer steps through the rate is governed by the use of the demand previously discussed. 
Normally, the number of kilowatt hours sold in any one block of the energy rate is determined by the hours use of the peak demand.

As an illustrative example, consider the general service rate depicted in Exhibit $I-5$ and an average commercial customer with the following electrical parameters :

- A summer peak of $500 \mathrm{~kW}$ for 4 months a year and an average demand of $350 \mathrm{~kW}$ for the remaining 8 months

- A summer energy consumption of $170,000 \mathrm{kWh}$ for 4 months and an average consumption of $110,000 \mathrm{kivh}$. for the remaining 8 months.

Based on the following scenarios; the cost effectiveness of storage cooling can be demonstrated.

- Scenario 1 - Calculated on the general service rate with no stored cooling

- Scenario 2 - Calculated on the general service rate with 50 percent stored cooling

- Scenario 3 - Calculated on the general service rate with a racheted demand using no stored cooling.

- Scenario 4 - Calculated on the general service rate with a racheted demand and 50 percent stored cooling.

The results indicate that:

- There is a $\$ 2,049$ annual energy saving associated with the addition of 50 percent stored cooling to scenario 1

- There is a $\$ 4,070$ annual energy saving associated with adding the stored cooling to scenario 3 which has a racheted demand rate.

Depending on the individual commercial customer's demand and consumption, TES under the traditional rate structure may be cost effective. However, declining block energy rate structures with no demand component would not provide any benefit to residential customers since there is no demand rate incentive to shift their load. 


\section{GENERAL SERVICE RATE G-2}

Nailable for comercial and industrial uses at a single location where the service vollage is less than 5000 volts. Not available for resale or domestic service in residential premises:

Rate:

Demand Charge

During the billing months of:

$\frac{\text { July-October }}{5131.90} \frac{\text { November-June }}{5131.90}$

$\$ 131.90 \quad \frac{N 131.90}{20}$

$5.21 \quad 4.36$

per month for the first 20 kiluwatts of demand or any portion thereof.

$4.22 \quad 3.37$

$4.15 \quad 3.30$

per kilowate per month for the rext 130 kilowates of demand.

per kilowatt per month for the next 650 kilowates of demand.

per kilowatt per month for the excess

\section{Energy Charge}

2.20 cents per kilowatthour for the first 290 hours' use of the demand per month.

1.33 cents per. kilowatchour for the next 150 hours' use of the demand per month.

0.92 cents per kilowatthour for the excess.

During the billing months of July through October, all use will be billed an additional charge of

0.52 cents per k1lowatthour.

\section{Additional Energy Charge}

1.18 cents per kilowathour for all direct_current energy.

Euel and Purchased Power Adjustment as provided in "Fuel and Purchased Power Adjustment," applicable to all kilowatthours or this rate.

Deteimination of Demand: The maximum fifteen-minute demand (either kilovatts or 80 percent of the kilovolt-amperes) will be determined by meter during the monthly billing period except, any demand recorded between 12 p.p.* and 9 a.m.* and ali day Saturdey and Sunday will be reduced by i0 percent. The number of kilowacts of demand billed shall be the maximum demand so determined, but in mo case less than the higher of the Following: (I) 20 kilowates and (2) where Auxiliary Service is supplied,

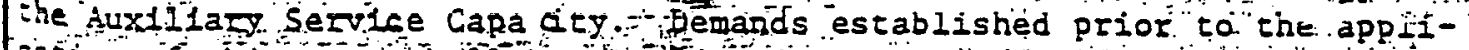
sation of this rate shali be considered as haviag been estabisished under sis rate.

*:Ll times are Eastern Daylight Savings Time and pertain to the sumer offpeak period. Corresponding Eastern Standard Times are 11 p.m. and 8 a.m. 
(2) The Inverted Declining Block Rate Structures Would Encourage Commercial Customers to Use TES, But Again There Would Be No Incentive for Residential Customers

The inverted declining block rate structure is the same as the traditional declining block in all aspects except one, the method of pricing each successive step. The energy and demand per unit costs increase with each successive step.

Inverted declining block rates are not necessarily in line with PURPA, which is designed to guide the utilities in ratemaking. In essence, the act requires that utilities charge the customer the true or marginal cost to produce energy. The next section will look at one such rate which marginally prices energy.

The Time of Use or Time of Day Rates Will Provide the Commercial Customers-and to a Lesser Extent the Residential Customers--with the Necessary Incentives to Install TES

The time of day (TOD) rate concept is to price both the energy and demand rate components to reflect their true cost for that particular time of the day. Time of use (TOU) rates are the same as TOD with one additional dimension, a cost factor for seasonal load variations.

Both forms of rates would provide substantial. cost differentials between the on-peak and off-peak rates. In conversations with several utilities experimenting with TOU rates, we found differentials ranging from 3 to 1 , to 7 to 1 . However, the fuel adjustment appears to be holding back the TES penetration, because of its additive effect to the energy cost portion of the rates. This adjustment degrades the payback residential customers expect from their investment. Commercial customers are also charged a TOU demand rate which offsets the negative impact of the fuel adjustment.

In general, the residential class of customers will not be able to achieve the same level of cost benefits that the commercial class will, with the possible exception in the area of domestic water heating. Domestic water heating makes up a greater portion of the residential load. 
Electric utilities hold a unique position as primemovers in encouraging the widespread application of TES. Electric utility involvement with TES will instill confidence in the viability of these systems with the suppliers and contractors--as well. as with the residential and commercial end-users.

Presently, electric utilities generally have a positive but cautious attitude towards TES. They believe that the technical expertise exists and that the industry infrastructure will mature and grow as the market develops. Further, the utilities' experience with the end user is that customer acceptance is generally very good, while customer awareness is relatively poor. Thus, the majority are taking a "wait-and-see" posture and are waiting for the results of several major demonstration efforts before initiating large scale TES projects. With few exceptions-notably American Electric Power and several Vermont utilities--the utility industry feels that significant TES deployment is at least five to ten years away.

Chapter II discusses the size of the economic market from the end-user's perspective. 


\section{ECONOMIC MARKET FROM END USERS' PERSPECTIVE}

The requirements for installing new space heating, water heating and air conditioning units in new construction, and retrofit buildings creates a large potential market for TES. For economic reasons, TES is only competitive with a portion of this market. The size of the economic market is principally constrained by the current utility rate designs.

Based on comparing the life cycle costs of TES and conventional alternatives, the economic attractiveness of TES is determined from the perspective of the residential and commercial customer.* The methodology for the lifecycle cost analysis is explained in Appendix A. A summary of the assumptions and results of the calculations is presented in Appendix B.

While the economic feasibility of TES is highly situation-specific, the economic analysis indicated that TES is generally an attractive option in large parts of the residential and commercial markets having high usage of electrical and oil-burning units. The size of the economic market is potentially large, based on considerations of the following three factors:

- Regionally-unique parameters

- Alternative fuel types

- End-use applications

The remainder of this chapter discusses these factors in greater detail.

1. IN MOST PARTS OF UNITED STATES, SOME TES TECHNOLOGY IS ECONOMICALIY COMPETITIVE IN BOTH RESIDENTIAL AND COMMERCIAI MARKETS

The domestic market for TES technology considered ready for early commercialization has been described in

The industrial sector application of TES generaliy involves other TES technologies used to recover process heat and is outside the scope of this study. 
several reports and and articles.3-6 The Phase II report of the Commercialization Task Force on Distributed Thermal Energy Storage and other documents have presented data on the cost and performance of storage space heating, storage air conditioning and storage water heating, indicating these are viable options in the residential market. The general conclusions of the task force report are:

- Storage resistance heating is applicable to residential electric space heating markets served by winter peaking utilities.

- Storage air conditioning is applicable to residential markets served by summer. peaking utilities

- Storage hot water heating is applicable to the entire, domestic residential water heating market.

The comparative economics of TES and conventional systems in different parts of the United states varies with regional rate structures, cost of energy and energy use. Using four year payback as the key customer economic decision making criterion, a rate differential between on-peak and off-peak prices of electricity was calculated. Exhibit II-l shows the rate differentials between onpeak and off-peak electricity prices for residential TES in different DOE regions:

- TES space heating rate differentials range from 3.3 to $9.34 / \mathrm{Kwh}$.

- TES water heating requires about a $2 \xi /$ Kwh differential.

- TES air conditioning rates require a minimum differential of $11.4 \mathrm{k} / \mathrm{kwh}$.

Some of these rate differentials could be offered under existing rate designs, while others are sufficiently large to require a change in current rate designs. Presently, utility rates favorable to TES are in effect or have been filed for consideration in 32 states. 7 However, many of these rate structures are still experimental. 


$$
\text { EXHIBIT II- } 1
$$

Economic Competitiveness of

TES and Conventional Systems

in the Residential sector

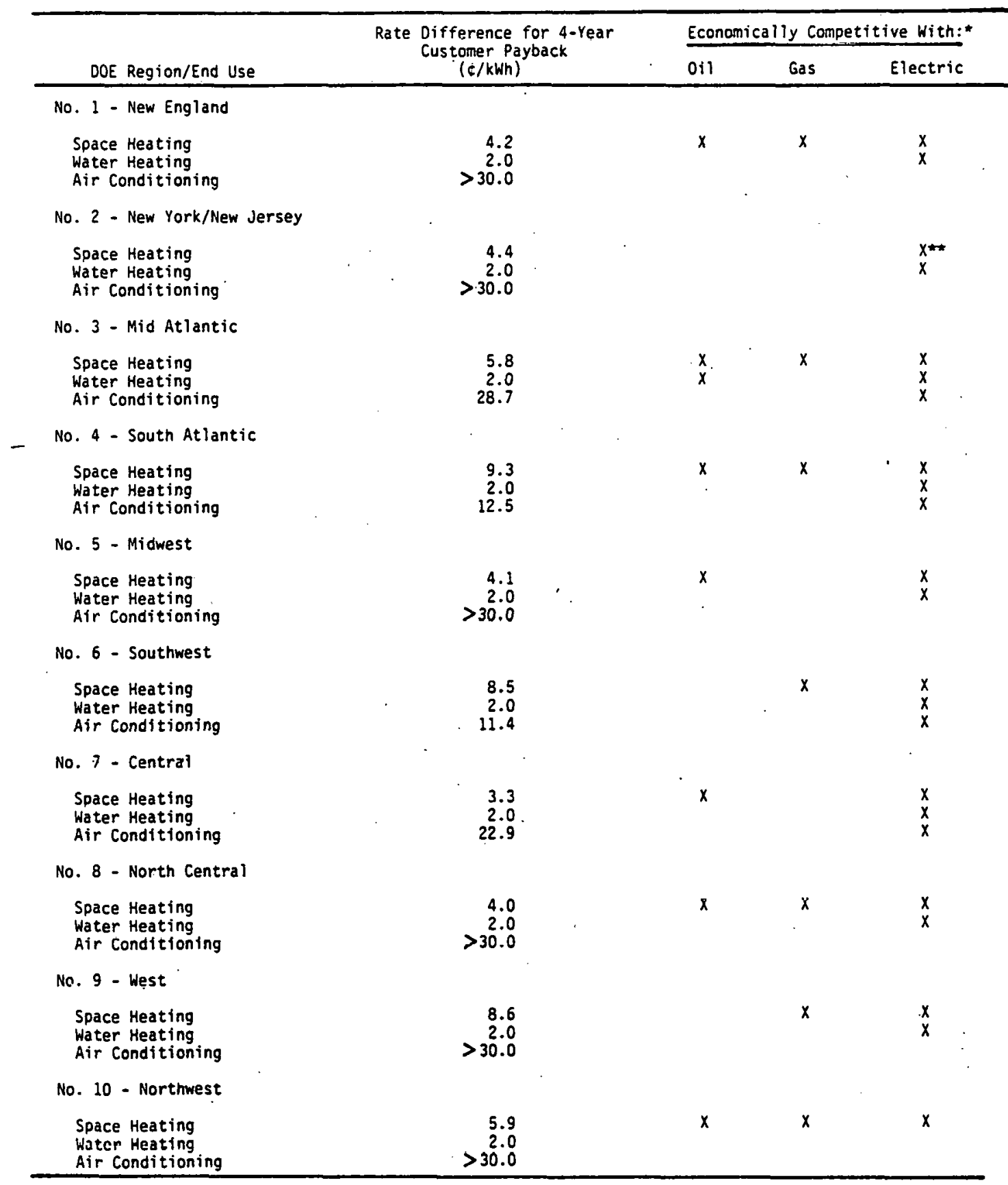

* Based on comparative life-cycle costs

** Electric Resistance Heating Oniy 
Assuming that a rate differential is available and sufficiently large to allow the customer a four-year payback, these TES technologies become competitive on a lifecycle cost basis with most conventional electric equipment. TES space heating also is economical compared to oil-burning systems in most regions. Despite the economic advantage of TES compared to some heat pumps and gasfired systems, however, the private sector perceives that TES will not displace these systems very significantly.

In commercial buildings, based on a review of recent studies of electricity use for thermal services, Heitner ${ }^{3}$ concludes:

- Generally, there is little or no market for TES in commercial space heating.

- Commercial air conditioning represents a large thermal load where TES systems could be used to improve the system load profiles in summer peaking utilities.

- In some utility areas, additional potential exists for TES water heating systems.

Calculations of the relative economics of TES and conventional systems in the commercial market introduces an added level of complexity resulting from the diversity of building types and energy use within these building types. Although data for building types in various regions of the country is available from regional bulletins of the Dodge Construction Potentials ${ }^{8}$, this study restricted its analysis to representative building types-office building, schools and retail stores. As shown in Exhibit II-2 these three types of buildings constitute about 57 percent of the commercial floor space, 6? percent of air conditioning use and 51 percent of water heating use. Estimates of the rate differentlal required to achieve a four-year payback for a $70,000 \mathrm{ft}^{2}$ retail store range did not exceed $2 \xi / K w h$, as shown in Exhibit II-3. Such rates result in TES being economically competitive on a life cycle cost basis with conventional electrical systems. The life cycle costs of oil and gas units were not calculated, because the private sector indicated that few new commercial buildings would be constructed with oil-burning systems and that gas units would be preferred over TES systems in most cases. In addition, heat pumps would be a principal competing technology to TES. 


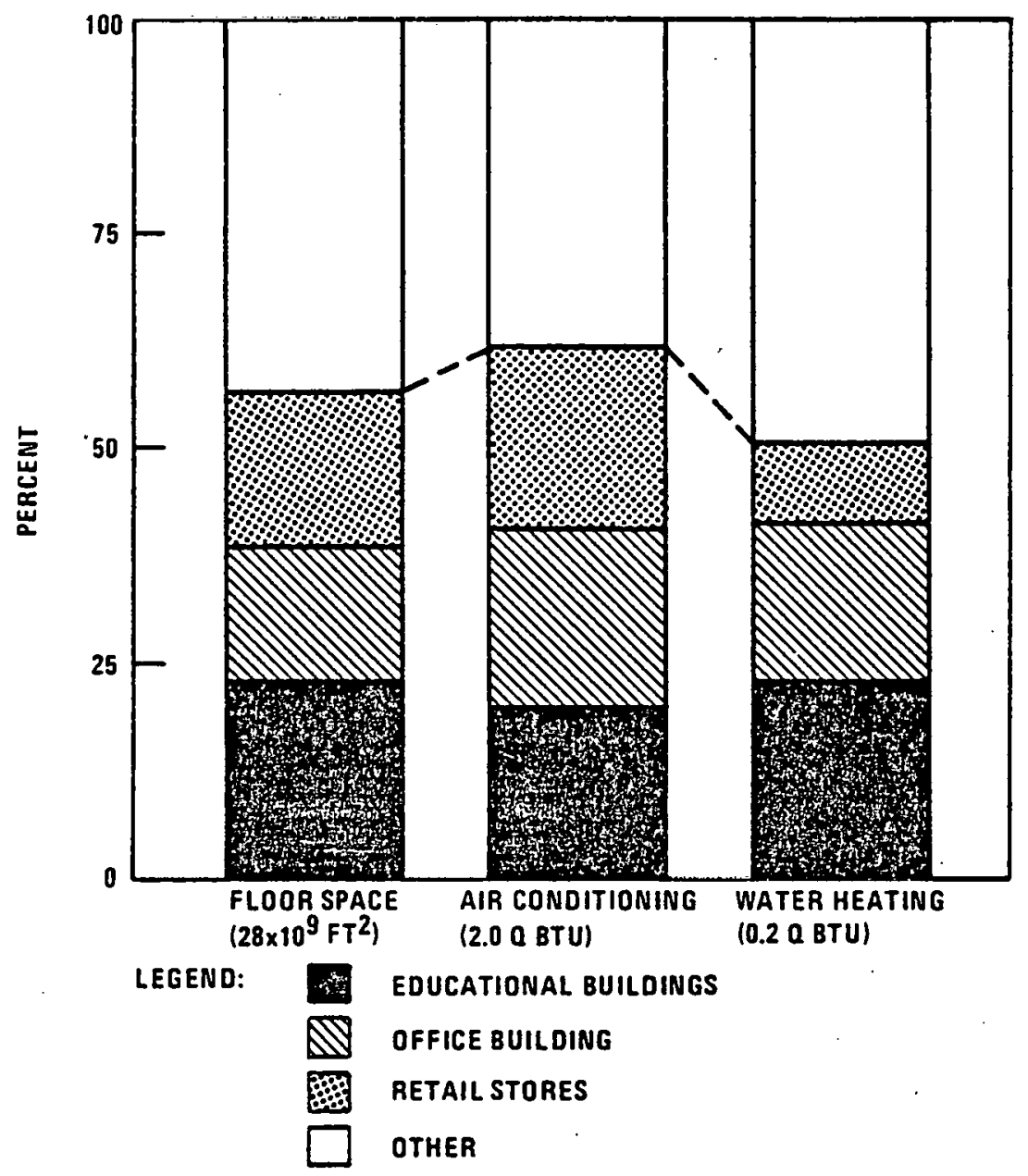

SOURCE: JACKSON, JERRY R. ANO WILLIAM S. JOHNSON, COMMERCIAL ENERGY USE: A DISAGGAEGATION BY FUEL, BUILDING TYPE, AND END USE, OAK RIDGE NATIONAL LABORATORY, ORNL/CON-14, FEBRUARY 1978. BASEO ON 1975 DATA. 


\section{EXHIBIT II-3}

Economic Competitiveness of TES

and Conventional systems

in the commercial sector

\begin{tabular}{|c|c|c|}
\hline Building Type/End Use & $\begin{array}{l}\text { Rate for } 4-Y e a r \\
\text { Customer Payback } \\
(\$ / \mathrm{kWh})\end{array}$ & $\begin{array}{l}\text { Economically Competitive } \\
\text { With Conventional } \\
\text { Electric System }\end{array}$ \\
\hline \multicolumn{3}{|l|}{ Office Building } \\
\hline $\begin{array}{l}\text { Water Heating } \\
\text { Air Conditioning }\end{array}$ & $\begin{array}{l}0.4 \\
1.5\end{array}$ & $\begin{array}{l}x \\
X\end{array}$ \\
\hline \multicolumn{3}{|l|}{ Educational Building } \\
\hline $\begin{array}{l}\text { Water Heating } \\
\text { Air Conditioning }\end{array}$ & $\begin{array}{l}1.0 \\
1.4\end{array}$ & $\begin{array}{l}x \\
x\end{array}$ \\
\hline \multicolumn{3}{|l|}{ Retail Store } \\
\hline $\begin{array}{l}\text { Water Heating } \\
\text { Air Conditioning }\end{array}$ & $\begin{array}{l}N C \star \star \\
1.9\end{array}$ & $\begin{array}{l}x \\
x\end{array}$ \\
\hline
\end{tabular}


2. COMPARED TO OTHER FUEL TYPES, TES IS ECONOMICAL WITH MOST CONVENTIONAL SPACE HEATING, WATER HEATING AND AIR CONDITIONING SYSTEMS

If TES were to displace all the new installations with which it is economic, TES could be used for over $200 \mathrm{GW}$ of demand. The TES demand included both installation in new construction and retrofit units for obsolete equipment that had to be replaced. As shown in Exhibit II-4, the demand that could be supplied by TES is attributed principally to displacing electric systems. TES could account for over 90 percent of electric systems and nearly 70 percent of oilfired systems demand. TES space heating units are economic with just a little more than 25 percent of the gas furnaces.

Demand was used as a measure of the TES market, because utilities will promote TES to displace load and save capacity. Residential demand was based on the number of new space heating, water heating and air conditioning installations in single and multi-family housing, as projected by Oak Ridge National laboratory. 9 These are summarized in Appendix $C$ for reference. Mobile homes constitute only about five percent of the housing market. Because they are a small part of the market and have little space that can be dedicated to TES equipment, mobile homes are not considered in this analysis. The average demands for each end-use in both single and multi-family housing for the 10 DOE regions, were calculated from ASHRAE data and are presented in Appendix $D$. The new space heating, water heating and air conditioning demand in residential single and multifamily housing is summarized in Exhibits II-5, 6, and 7, respectively.

In the commercial sector, demand for water heating and air conditioning was estimated from projections of construction of additional commercial floor space for the three building types 10 and the average demand for a typical building in these type categories. The demand results were allocated among the various DOE regions, and are summarized for the two comnercial applications in Exhibits II-8, and II 9 . 
EXHIBIT II-4

TES Market By Fuel Type

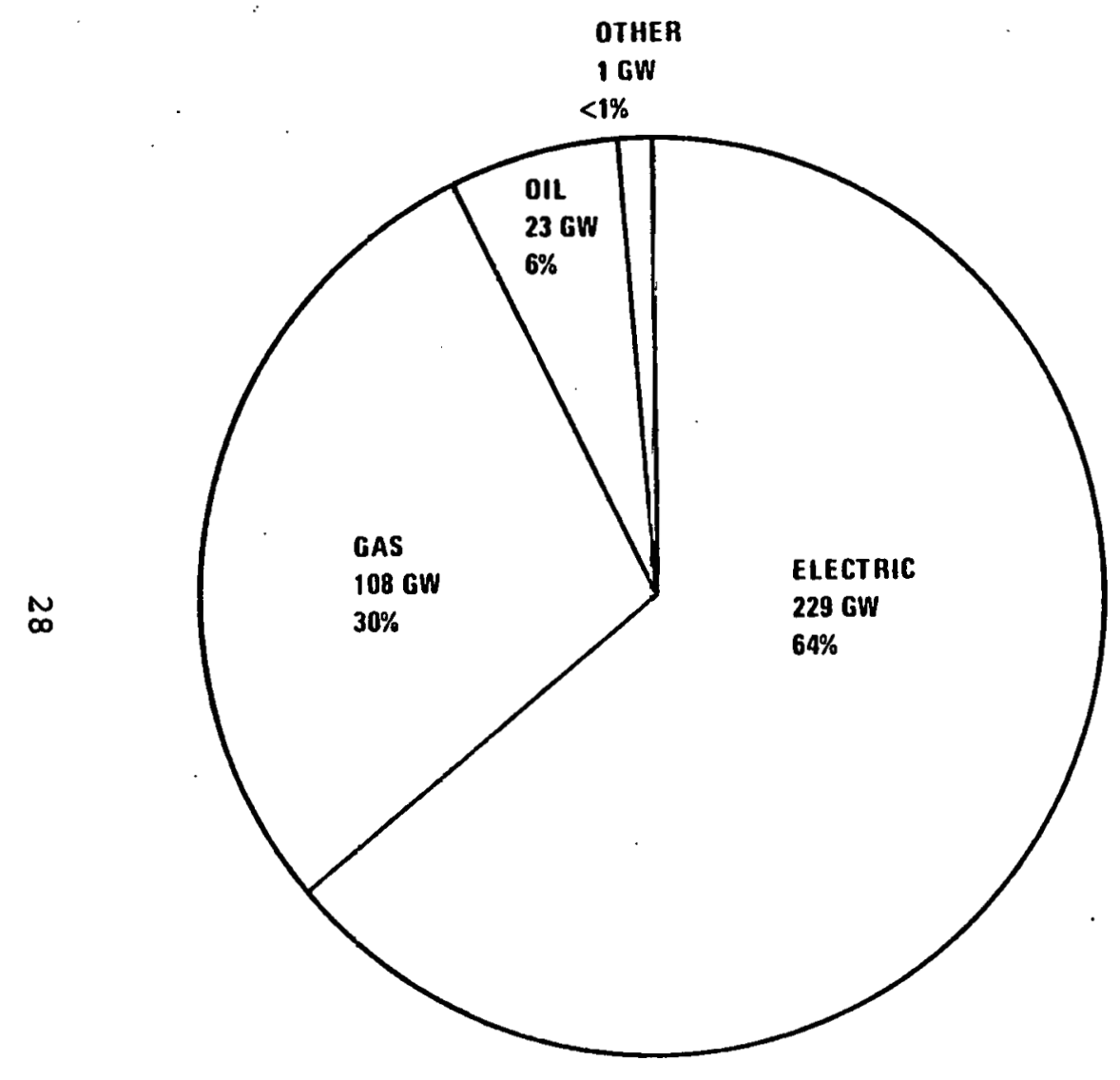

TOTAL RESIDENTIAL AND COMMERCIAL

DEMAND FOR SPACE HEATWG, WATER HEATING AND AIR CONDITIONING $360 \mathrm{GW}$

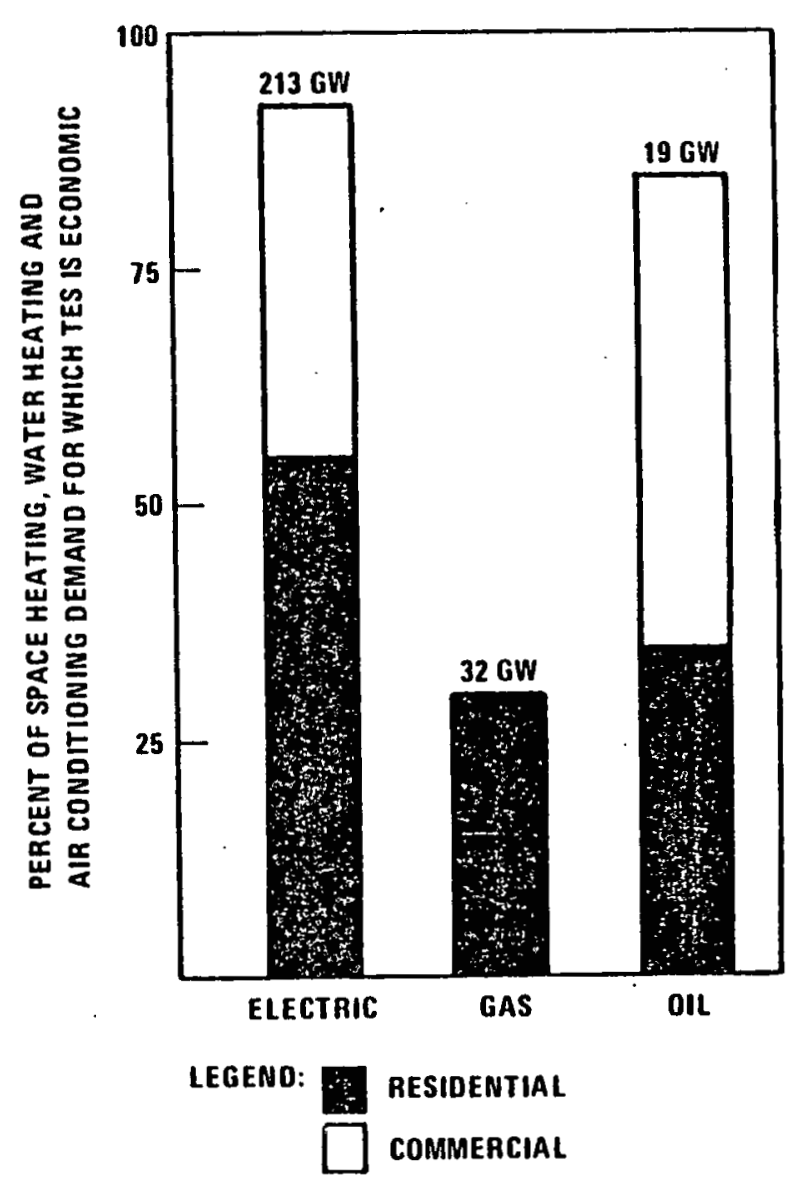

- based on comparative life.cYcle costs 
EXHIBIT II-5

New Space Heating Demand

in Single and Multifamily Housing

(Gigawatts)

\begin{tabular}{|c|c|c|c|c|c|c|c|c|c|c|}
\hline \multirow[b]{2}{*}{ No. 1 - New Engl and } & \multicolumn{2}{|c|}{$\begin{array}{c}1980 \\
\text { Single Multi }\end{array}$} & \multicolumn{2}{|c|}{$\begin{array}{c}1985 \\
\text { Single Multi }\end{array}$} & \multicolumn{2}{|c|}{$\begin{array}{l}1990 \\
\text { Single Muit }\end{array}$} & \multicolumn{2}{|c|}{$\begin{array}{l}1995 \\
\text { Single Multi }\end{array}$} & \multicolumn{2}{|c|}{$\begin{array}{l}2000 \\
\text { Singie Multi }\end{array}$} \\
\hline & & & & & & & & & & \\
\hline $\begin{array}{l}\text { Electric } \\
\text { Gas } \\
\text { Oil } \\
\text { Total }\end{array}$ & $\begin{array}{l}.61 \\
1.57 \\
2.80 \\
4.98\end{array}$ & $\begin{array}{l}.17 \\
.37 \\
.32 \\
.86\end{array}$ & $\begin{array}{l}.73 \\
1.61 \\
3.19 \\
5.53\end{array}$ & $\begin{array}{l}.19 \\
.38 \\
.37 \\
.94\end{array}$ & $\begin{array}{l}.81 \\
1.69 \\
3.30 \\
5.80\end{array}$ & $\begin{array}{l}.20 \\
.39 \\
.37 \\
.96\end{array}$ & $\begin{array}{l}1.15 \\
2.23 \\
2.53 \\
5.91\end{array}$ & $\begin{array}{l}.26 \\
.46 \\
.26 \\
.98\end{array}$ & $\begin{array}{l}1.80 \\
2.80 \\
1.46 \\
6.06\end{array}$ & $\begin{array}{r}.35 \\
.52 \\
.13 \\
1.00\end{array}$ \\
\hline \multicolumn{11}{|l|}{ No, 2 - New York/New Jersey } \\
\hline $\begin{array}{l}\text { Electric } \\
\text { Gas } \\
\text { Ofl } \\
\text { Total }\end{array}$ & $\begin{array}{l}.35 \\
3.83 \\
2.27 \\
6.45\end{array}$ & $\begin{array}{r}.13 \\
.79 \\
.82 \\
1.74\end{array}$ & $\begin{array}{l}.43 \\
4.01 \\
2.41 \\
6.85\end{array}$ & $\begin{array}{r}.14 \\
.82 \\
.85 \\
1.81\end{array}$ & $\begin{array}{l}.46 \\
3.94 \\
2.48 \\
6.88\end{array}$ & $\begin{array}{r}.14 \\
.76 \\
.84 \\
1.74\end{array}$ & $\begin{array}{l}.64 \\
4.18 \\
1.91 \\
6.73\end{array}$ & $\begin{array}{r}.19 \\
.83 \\
.70 \\
1.72\end{array}$ & $\begin{array}{l}.92 \\
4.43 \\
1.24 \\
6.59\end{array}$ & $\begin{array}{r}.27 \\
.89 \\
.50 \\
i .66\end{array}$ \\
\hline \multicolumn{11}{|l|}{ No. 3 - Mid Atlantic } \\
\hline $\begin{array}{l}\text { Electric } \\
\text { Gas } \\
\text { ofl } \\
\text { Total }\end{array}$ & $\begin{array}{r}2.26 \\
6.38 \\
3.33 \\
11.97\end{array}$ & $\begin{array}{l}.27 \\
.47 \\
.21 \\
.95\end{array}$ & $\begin{array}{r}2.57 \\
6.17 \\
3.43 \\
12.17\end{array}$ & $\begin{array}{l}.29 \\
.46 \\
.22 \\
.97\end{array}$ & $\begin{array}{r}2.81 \\
6.10 \\
3.43 \\
12.34\end{array}$ & $\begin{array}{l}.30 \\
.44 \\
.22 \\
.96\end{array}$ & $\begin{array}{r}3.29 \\
6.17 \\
2.85 \\
12.31\end{array}$ & $\begin{array}{l}.33 \\
.44 \\
.18 \\
.95\end{array}$ & $\begin{array}{r}4.01 \\
6.17 \\
2.16 \\
12.34\end{array}$ & $\begin{array}{l}.37 \\
.43 \\
.14 \\
.94\end{array}$ \\
\hline \multicolumn{11}{|l|}{ No. 4-South Atlantic } \\
\hline $\begin{array}{l}\text { Electric } \\
\text { Gas } \\
\text { Oil } \\
\text { Total }\end{array}$ & $\begin{array}{r}12.98 \\
5.16 \\
1.71 \\
19.85\end{array}$ & $\begin{array}{l}.65 \\
.24 \\
.03 \\
.92\end{array}$ & $\begin{array}{r}13.98 \\
4.57 \\
1.74 \\
20.29\end{array}$ & $\begin{array}{l}.67 \\
.23 \\
.03 \\
.93\end{array}$ & $\begin{array}{r}14.81 \\
4.75 \\
1.77 \\
21.33\end{array}$ & $\begin{array}{l}.68 \\
.25 \\
.03 \\
.96\end{array}$ & $\begin{array}{r}15.62 \\
4.97 \\
1.71 \\
22.30\end{array}$ & $\begin{array}{r}.70 \\
.27 \\
.03 \\
1.00\end{array}$ & $\begin{array}{r}16.40 \\
5.19 \\
1.71 \\
23.30\end{array}$ & $\begin{array}{r}.72 \\
.28 \\
.03 \\
1.03\end{array}$ \\
\hline \multicolumn{11}{|l|}{ No. 5 - Midwest } \\
\hline $\begin{array}{l}\text { Electric } \\
\text { Gas } \\
\text { oil } \\
\text { Total }\end{array}$ & $\begin{array}{r}3.49 \\
19.30 \\
3.14 \\
25.93\end{array}$ & $\begin{array}{l}.63 \\
1.50 \\
.10 \\
2.23\end{array}$ & $\begin{array}{r}4.23 \\
19.03 \\
3.80 \\
27.06\end{array}$ & $\begin{array}{l}.69 \\
1.48 \\
.12 \\
2.29\end{array}$ & $\begin{array}{r}4.63 \\
18.38 \\
4.37 \\
27.38\end{array}$ & $\begin{array}{l}.70 \\
1.42 \\
.14 \\
2.26\end{array}$ & $\begin{array}{r}5.68 \\
17.16 \\
4.54 \\
29.38\end{array}$ & $\begin{array}{r}.79 \\
1.31 \\
.14 \\
2.24\end{array}$ & $\begin{array}{r}7.47 \\
15.32 \\
4.58 \\
27.37\end{array}$ & $\begin{array}{l}.93 \\
1.15 \\
.14 \\
2.22\end{array}$ \\
\hline \multicolumn{11}{|l|}{ No. 6 - Southwest } \\
\hline $\begin{array}{l}\text { Electric } \\
\text { Gas } \\
\text { Oii } \\
\text { Total }\end{array}$ & $\begin{array}{r}4.14 \\
8.08 \\
0 \\
12.22\end{array}$ & $\begin{array}{r}.37 \\
.17 \\
0 \\
.54\end{array}$ & $\begin{array}{r}5.45 \\
6.89 \\
0 \\
12.34\end{array}$ & $\begin{array}{r}.43 \\
.13 \\
0 \\
.56\end{array}$ & $\begin{array}{r}7.77 \\
4.97 \\
0 \\
12.74\end{array}$ & $\begin{array}{r}.49 \\
.08 \\
0 \\
.57\end{array}$ & $\begin{array}{r}10.42 \\
2.96 \\
0 \\
13.38\end{array}$ & $\begin{array}{r}.55 \\
.05 \\
0 \\
.60\end{array}$ & $\begin{array}{r}12.80 \\
1.43 \\
0 \\
14.23\end{array}$ & $\begin{array}{r}.59 \\
.03 \\
0 \\
.62\end{array}$ \\
\hline \multicolumn{11}{|l|}{ No. 7 - Central } \\
\hline $\begin{array}{l}\text { Electric } \\
\text { Gas } \\
011 \\
\text { Total }\end{array}$ & $\begin{array}{l}.79 \\
5.70 \\
.32 \\
6.80\end{array}$ & $\begin{array}{l}.08 \\
.28 \\
.01 \\
.37\end{array}$ & $\begin{array}{l}.95 \\
5.58 \\
.36 \\
6.89\end{array}$ & $\begin{array}{l}.08 \\
.28 \\
.01 \\
.37\end{array}$ & $\begin{array}{l}1.15 \\
5.22 \\
.51 \\
6.88\end{array}$ & $\begin{array}{l}.08 \\
.27 \\
.01 \\
.36\end{array}$ & $\begin{array}{l}1.62 \\
4.51 \\
.67 \\
6.80\end{array}$ & $\begin{array}{l}.09 \\
.25 \\
.01 \\
.35\end{array}$ & $\begin{array}{l}2.57 \\
3.24 \\
.91 \\
6.72\end{array}$ & $\begin{array}{l}.12 \\
.22 \\
.01 \\
.35\end{array}$ \\
\hline \multicolumn{11}{|l|}{ No. 8 - North Central } \\
\hline $\begin{array}{l}\text { Electric } \\
\text { Gas } \\
\text { ofi } \\
\text { Total }\end{array}$ & $\begin{array}{r}.34 \\
3.70 \\
.43 \\
4.47\end{array}$ & $\begin{array}{l}.05 \\
.22 \\
.01 \\
.28\end{array}$ & $\begin{array}{l}.52 \\
3.19 \\
.78 \\
4.49\end{array}$ & $\begin{array}{l}.06 \\
.21 \\
.01 \\
.28\end{array}$ & $\begin{array}{r}.65 \\
3.06 \\
.95 \\
4.66\end{array}$ & $\begin{array}{l}.06 \\
.20 \\
.02 \\
.28\end{array}$ & $\begin{array}{l}.95 \\
2.71 \\
1.16 \\
4.82\end{array}$ & $\begin{array}{l}.09 \\
.18 \\
.02 \\
.29\end{array}$ & $\begin{array}{l}1.46 \\
2.15 \\
1.38 \\
4.89\end{array}$ & $\begin{array}{l}.12 \\
.15 \\
.03 \\
.30\end{array}$ \\
\hline \multicolumn{11}{|l|}{ No. 9 - West } \\
\hline $\begin{array}{l}\text { Electric } \\
\text { Gas } \\
\text { o11 } \\
\text { Total }\end{array}$ & $\begin{array}{r}1.97 \\
4.39 \\
0 \\
6.36\end{array}$ & $\begin{array}{r}.36 \\
.27 \\
0 \\
.63\end{array}$ & $\begin{array}{l}3.89 \\
2.51 \\
0 \\
6.40\end{array}$ & $\begin{array}{l}.52 \\
.13 \\
0 \\
.65\end{array}$ & $\begin{array}{l}4.91 \\
1.87 \\
0 \\
6.78\end{array}$ & $\begin{array}{l}.57 \\
.10 \\
0 \\
.67\end{array}$ & $\begin{array}{l}4.89 \\
2.24 \\
0 \\
7.13\end{array}$ & $\begin{array}{r}.58 \\
.13 \\
0 \\
.71\end{array}$ & $\begin{array}{l}4.71 \\
2.79 \\
0 \\
7.50\end{array}$ & $\begin{array}{r}.57 \\
.16 \\
0 \\
.73\end{array}$ \\
\hline \multicolumn{11}{|l|}{ No. 10 - Northwest } \\
\hline $\begin{array}{l}\text { Electric } \\
\text { Gas } \\
\text { O1I } \\
\text { Total }\end{array}$ & $\begin{array}{r}2.17 \\
.71 \\
.15 \\
3.03\end{array}$ & $\begin{array}{r}.13 \\
.03 \\
<.01 \\
.16\end{array}$ & $\begin{array}{r}2.24 \\
.55 \\
.15 \\
2.94\end{array}$ & $\begin{array}{r}.13 \\
.03 \\
8.01 \\
.16\end{array}$ & $\begin{array}{r}2.32 \\
.58 \\
.18 \\
3.08\end{array}$ & $\begin{array}{r}.13 \\
.03 \\
<.01 \\
.16\end{array}$ & $\begin{array}{r}2.37 \\
.63 \\
.15 \\
.3 .15\end{array}$ & $\begin{array}{r}.13 \\
.03 \\
<.01 \\
.16\end{array}$ & $\begin{array}{r}2.42 \\
.68 \\
.10 \\
3.20\end{array}$ & $\begin{array}{r}.13 \\
.04 \\
<.01 \\
.17\end{array}$ \\
\hline \multicolumn{11}{|l|}{ United States } \\
\hline $\begin{array}{l}\text { Electric } \\
\text { Gas } \\
011 \\
\text { Total }\end{array}$ & $\begin{array}{r}29.10 \\
58.82 \\
14.15 \\
102.06\end{array}$ & $\begin{array}{l}2.84 \\
4.34 \\
1.50 \\
8.68\end{array}$ & $\begin{array}{r}34.99 \\
54.11 \\
15.86 \\
104.96\end{array}$ & $\begin{array}{l}3.20 \\
4.15 \\
1.61 \\
8.95\end{array}$ & $\begin{array}{r}40.32 \\
50.56 \\
16.99 \\
107.87\end{array}$ & $\begin{array}{l}3.35 \\
3.94 \\
1.63 \\
8.92\end{array}$ & $\begin{array}{r}46.63 \\
47.76 \\
15.52 \\
109.91\end{array}$ & $\begin{array}{l}3.71 \\
3.95 \\
1.34 \\
8.00\end{array}$ & $\begin{array}{r}94.56 \\
44.20 \\
13.54 \\
112.30\end{array}$ & $\begin{array}{l}4.17 \\
3.87 \\
.98 \\
9.02\end{array}$ \\
\hline
\end{tabular}

Total demand was based on new installed units as projected in Residential

Energy Use Simulations. 1970-2000, Version 6 (Decenber 1978). Oak Ridge

Energy Use Simulations, $1970-2000$, Version 6 (Decenber 1978). Oak Ridde
National Laborstory, and average denand per unit for each region, as

National Laboratory, and aver from ASHRAE data. 


\begin{tabular}{|c|c|c|c|c|c|}
\hline Dot Region/fuel Type & 7980 & 1985 & 1990 & 1995 & 2000 \\
\hline \multicolumn{6}{|l|}{ No. 1 - Hew England } \\
\hline $\begin{array}{l}\text { Electric } \\
\text { Gas } \\
\text { Oil } \\
\text { Total }\end{array}$ & $\begin{array}{r}1.01 \\
.81 \\
.14 \\
1.96\end{array}$ & $\begin{array}{r}1.11 \\
.94 \\
.16 \\
2.21\end{array}$ & $\begin{array}{r}1.17 \\
.92 \\
.15 \\
2.24\end{array}$ & $\begin{array}{l}1.30 \\
1.00 \\
.05 \\
2.35\end{array}$ & $\begin{array}{r}1.38 \\
1.03 \\
.01 \\
2.42\end{array}$ \\
\hline \multicolumn{6}{|l|}{ Ho. 2 - New York/New Jersey } \\
\hline $\begin{array}{l}\text { Electric } \\
\text { Gas } \\
011 \\
\text { Total }\end{array}$ & $\begin{array}{r}.81 \\
2.55 \\
.29 \\
3.66\end{array}$ & $\begin{array}{r}.90 \\
2.63 \\
.32 \\
3.85\end{array}$ & $\begin{array}{r}1.04 \\
2.53 \\
.29 \\
3.86\end{array}$ & $\begin{array}{l}1.28 \\
2.46 \\
.09 \\
3.83\end{array}$ & $\begin{array}{r}1.54 \\
2.23 \\
.02 \\
3.79\end{array}$ \\
\hline \multicolumn{6}{|l|}{ No. 3 - Mid Atlantic } \\
\hline $\begin{array}{l}\text { Electric } \\
\text { Gas } \\
\text { Oil } \\
\text { Total }\end{array}$ & $\begin{array}{r}1.49 \\
1.87 \\
.19 \\
3.55\end{array}$ & $\begin{array}{l}1.58 \\
1.86 \\
.23 \\
3.67\end{array}$ & $\begin{array}{r}1.72 \\
1.80 \\
.23 \\
3.75\end{array}$ & $\begin{array}{l}1.86 \\
1.77 \\
.16 \\
3.79\end{array}$ & $\begin{array}{r}1.98 \\
1.74 \\
.10 \\
3.83\end{array}$ \\
\hline \multicolumn{6}{|l|}{ No. 4-South Atlantic } \\
\hline $\begin{array}{l}\text { Electric } \\
\text { Gas } \\
\text {. Total }\end{array}$ & $\begin{array}{l}3.99 \\
1.44 \\
5.43\end{array}$ & $\begin{array}{l}4.15 \\
1.48 \\
5.63\end{array}$ & $\begin{array}{l}4.37 \\
1.58 \\
5.95\end{array}$ & $\begin{array}{l}4.56 \\
1.68 \\
6.24\end{array}$ & $\begin{array}{l}4.75 \\
1.78 \\
6.53\end{array}$ \\
\hline \multicolumn{6}{|l|}{ No. 5 - Midwest } \\
\hline $\begin{array}{l}\text { Electric } \\
\text { Gas } \\
\text { Total }\end{array}$ & $\begin{array}{l}2.21 \\
4.22 \\
6.43\end{array}$ & $\begin{array}{l}2.87 \\
4.37 \\
7.24\end{array}$ & $\begin{array}{l}3.27 \\
4.00 \\
7.37\end{array}$ & $\begin{array}{l}3.82 \\
3.63 \\
7.45\end{array}$ & $\begin{array}{l}4.44 \\
3.05 \\
7.49\end{array}$ \\
\hline \multicolumn{6}{|l|}{ No. 6 - Soutmest } \\
\hline $\begin{array}{l}\text { Electric } \\
\text { Gas } \\
\text { Other } \\
\text { Tota } 1\end{array}$ & $\begin{array}{r}1.39 \\
1.43 \\
.17 \\
2.99\end{array}$ & $\begin{array}{r}2.21 \\
.82 \\
.11 \\
3.14\end{array}$ & $\begin{array}{r}2.97 \\
.27 \\
.07 \\
3.31\end{array}$ & $\begin{array}{r}3.37 \\
.06 \\
.02 \\
3.45\end{array}$ & $\begin{array}{r}3.59 \\
.01 \\
5.01 \\
3.60\end{array}$ \\
\hline \multicolumn{6}{|l|}{ No. 7 - Central } \\
\hline $\begin{array}{l}\text { Electrte } \\
\text { Gas } \\
\text { Other } \\
\text { Total }\end{array}$ & $\begin{array}{r}.55 \\
1.14 \\
.05 \\
1.74\end{array}$ & $\begin{array}{l}.70 \\
1.05 \\
.03 \\
1.78\end{array}$ & $\begin{array}{r}.97 \\
.81 \\
.02 \\
1.80\end{array}$ & $\begin{array}{r}1.24 \\
.56 \\
.01 \\
1.81\end{array}$ & $\begin{array}{r}1.46 \\
.34 \\
.01 \\
1.81\end{array}$ \\
\hline \multicolumn{6}{|l|}{ No. 8 - North Central } \\
\hline $\begin{array}{l}\text { Electric } \\
\text { Gas } \\
\text { Other } \\
\text { Total }\end{array}$ & $\begin{array}{l}.46 \\
.60 \\
.02 \\
1.08\end{array}$ & $\begin{array}{r}.62 \\
.48 \\
.02 \\
1.12\end{array}$ & $\begin{array}{r}.79 \\
.37 \\
.01 \\
1.17\end{array}$ & $\begin{array}{r}.98 \\
.23 \\
.01 \\
1.22\end{array}$ & $\begin{array}{l}1.16 \\
.11 \\
5.01 \\
1.27\end{array}$ \\
\hline \multicolumn{6}{|l|}{ No. 9 - West } \\
\hline $\begin{array}{l}\text { Electric } \\
\text { lias } \\
\text { Other } \\
\text { Total }\end{array}$ & $\begin{array}{l}1.34 \\
2.89 \\
.04 \\
3.67\end{array}$ & $\begin{array}{r}3.36 \\
.46 \\
.05 \\
3.87\end{array}$ & $\begin{array}{r}3.96 \\
.11 \\
.02 \\
4.09\end{array}$ & $\begin{array}{r}4.14 \\
.14 \\
.01 \\
4.29\end{array}$ & $\begin{array}{r}4.28 \\
.21 \\
.01 \\
4.50\end{array}$ \\
\hline \multicolumn{6}{|l|}{ No. 10 - Northmest } \\
\hline $\begin{array}{l}\text { Electric } \\
\text { Gas } \\
\text { Other } \\
\text { Total }\end{array}$ & $\begin{array}{r}.92 \\
.11 \\
4.01 \\
1.03\end{array}$ & $\begin{array}{r}.93 \\
.11 \\
<.01 \\
1.04\end{array}$ & $\begin{array}{r}.96 \\
.13 \\
<.01 \\
1.09\end{array}$ & $\begin{array}{r}.98 \\
.14 \\
5.01 \\
1.12\end{array}$ & $\begin{array}{r}.98 \\
.16 \\
<.01 \\
1.14\end{array}$ \\
\hline \multicolumn{6}{|l|}{ United States } \\
\hline $\begin{array}{l}\text { Electric } \\
\text { Gas } \\
011 \\
\text { Other } \\
\text { Total }\end{array}$ & $\begin{array}{r}14.17 \\
16.46 \\
.62 \\
.28 \\
.31 .54\end{array}$ & $\begin{array}{r}18.43 \\
14.20 \\
.71 \\
.21 \\
33.55\end{array}$ & $\begin{array}{r}21.22 \\
12.62 \\
.67 \\
.12 \\
34.63\end{array}$ & $\begin{array}{r}23.53 \\
11.67 \\
.30 \\
3.05 \\
35.55\end{array}$ & $\begin{array}{r}25.56 \\
10.66 \\
.13 \\
.02 \\
36.38\end{array}$ \\
\hline
\end{tabular}

Total demand was based on new installed units as projected in Residential Total demand was based on new-2000, Version 6 (December 1978), Oak Ridge
Energy (Ise Slmulations. 1970-200, Energy Use Simulations, $1970-2000$, Version 6 (December 1978), Oak Ridge
Mational Laboratory, and average demand per unit for each region, as calculated from ASHRAE data.

$\cdot$ 
EXHIBIT II 7

New Air Conditioning Demand

in Single and Multifamily Housing

(Gigawatts)

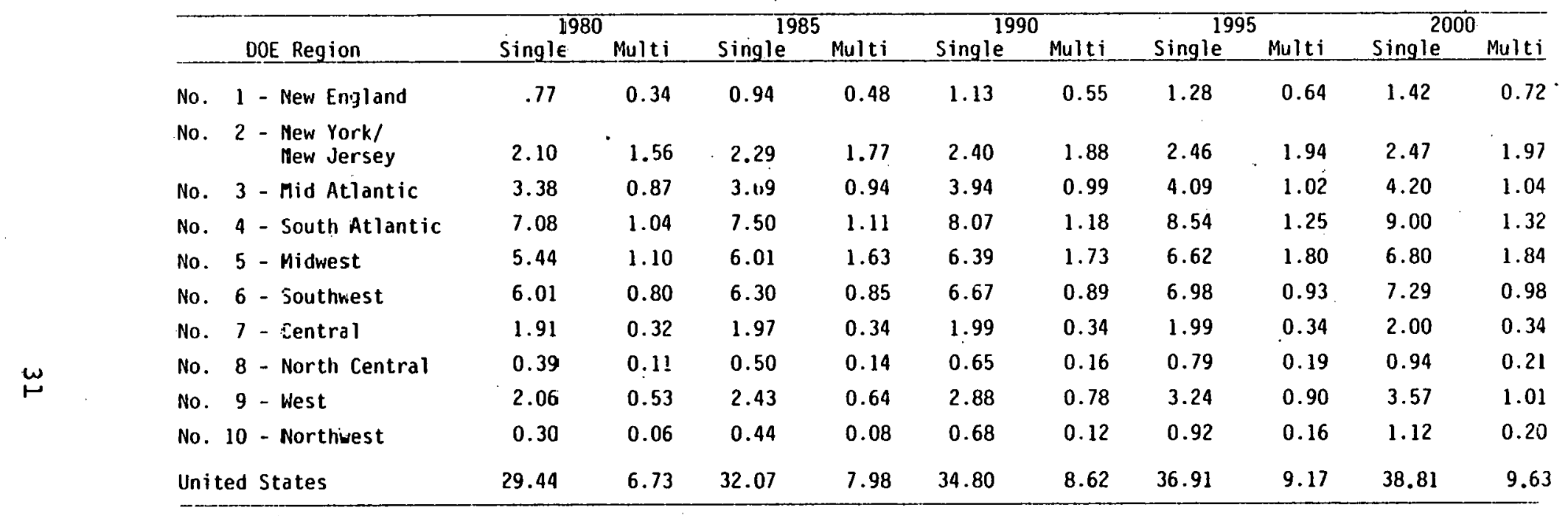

Total delaand was based on new installed units as projected in Residential Energy Use Simulations, 1970-2000,

Version 6 (December 1978), Oak Ridge National Laboratory, and average denand per unit for each region, as calculated from ASHRAE data. 


$$
\text { EXHIBIT II-8 }
$$

New Air Conditioning Demand in Commercial offices, Schools and Retail stores

(Gigawatts) I

\begin{tabular}{|c|c|c|c|c|c|}
\hline DOE REGION/RUEL TYPE & 1980 & 1985 & 1990 & 1995 & 2000 \\
\hline \multicolumn{6}{|l|}{ No. 1 - New England } \\
\hline $\begin{array}{l}\text { Electric } \\
\text { Gas } \\
\text { Total }\end{array}$ & $\begin{array}{l}2.9 \\
0.5 \\
3.4\end{array}$ & $\begin{array}{l}3.1 \\
0.5 \\
3.6\end{array}$ & $\begin{array}{l}3.3 \\
0.6 \\
3.9\end{array}$ & $\begin{array}{l}3.5 \\
0.7 \\
4.2\end{array}$ & $\begin{array}{l}3.9 \\
0.8 \\
4.7\end{array}$ \\
\hline \multicolumn{6}{|c|}{ No. 2 - New York/New Jersey } \\
\hline $\begin{array}{l}\text { Electric } \\
\text { Gas } \\
\text { Total }\end{array}$ & $\begin{array}{l}5.7 \\
1.0 \\
6.7\end{array}$ & $\begin{array}{l}6.2 \\
1.1 \\
7.3\end{array}$ & $\begin{array}{l}6.6 \\
1.2 \\
7.8\end{array}$ & $\begin{array}{l}7.1 \\
1.3 \\
8.4\end{array}$ & $\begin{array}{l}8.0 \\
1.6 \\
9.4\end{array}$ \\
\hline \multicolumn{6}{|l|}{ No. 3 - Mid Atlantic } \\
\hline $\begin{array}{l}\text { Electric } \\
\text { Gas } \\
\text { Total }\end{array}$ & $\begin{array}{r}21.2 \\
3.8 \\
25.0\end{array}$ & $\begin{array}{r}23.0 \\
3.9 \\
27.1\end{array}$ & $\begin{array}{r}25.0 \\
4.4 \\
29.4\end{array}$ & $\begin{array}{r}26.8 \\
4.7 \\
31.5\end{array}$ & $\begin{array}{r}29.8 \\
5.3 \\
35.1\end{array}$ \\
\hline \multicolumn{6}{|l|}{ No. 4 - South Atlantic } \\
\hline $\begin{array}{l}\text { Electric } \\
\text { Gas } \\
\text { Total }\end{array}$ & $\begin{array}{r}29.7 \\
5.3 \\
35.0\end{array}$ & $\begin{array}{r}32.3 \\
5.7 \\
38.0\end{array}$ & $\begin{array}{r}35.1 \\
6.2 \\
41.3\end{array}$ & $\begin{array}{r}37.5 \\
6.6 \\
44.1\end{array}$ & $\begin{array}{r}41.6 \\
7.5 \\
49.1\end{array}$ \\
\hline \multicolumn{6}{|l|}{ No. 5 - Midwest } \\
\hline $\begin{array}{l}\text { Electric } \\
\text { Gas } \\
\text { Total }\end{array}$ & $\begin{array}{r}16.9 \\
3.1 \\
20.0\end{array}$ & $\begin{array}{r}18.4 \\
3.3 \\
21.7\end{array}$ & $\begin{array}{r}20.0 \\
3.5 \\
23.5\end{array}$ & $\begin{array}{r}21.4 \\
3.8 \\
25.2\end{array}$ & $\begin{array}{r}23.9 \\
4.2 \\
28.1\end{array}$ \\
\hline \multicolumn{6}{|l|}{ No. 6 - Southeast } \\
\hline $\begin{array}{l}\text { Electric } \\
\text { Gas } \\
\text { Total }\end{array}$ & $\begin{array}{r}34.5 \\
3.9 \\
38.4\end{array}$ & $\begin{array}{r}37.3 \\
4.3 \\
41.6\end{array}$ & $\begin{array}{r}40.5 \\
4.6 \\
45.1\end{array}$ & $\begin{array}{r}43.4 \\
4.9 \\
48.3\end{array}$ & $\begin{array}{r}48.3 \\
5.5 \\
53.8\end{array}$ \\
\hline \multicolumn{6}{|l|}{ No. 7 - Central } \\
\hline $\begin{array}{l}\text { Electric } \\
\text { Gas } \\
\text { Total }\end{array}$ & $\begin{array}{l}4.5 \\
0.5 \\
5.0\end{array}$ & $\begin{array}{l}4.8 \\
0.6 \\
5.4\end{array}$ & $\begin{array}{l}5.1 \\
0.8 \\
59\end{array}$ & $\begin{array}{l}5.4 \\
0.9 \\
6.3\end{array}$ & $\begin{array}{l}6.0 \\
1.0 \\
7.0\end{array}$ \\
\hline \multicolumn{6}{|l|}{ No. 8 - North Central } \\
\hline $\begin{array}{l}\text { Electric } \\
\text { Gas } \\
\text { Total }\end{array}$ & $\begin{array}{l}4.3 \\
0.7 \\
5.0\end{array}$ & $\begin{array}{l}4.6 \\
0.8 \\
5.4\end{array}$ & $\begin{array}{l}5.0 \\
0.9 \\
5.9\end{array}$ & $\begin{array}{l}5.4 \\
0.9 \\
6.3\end{array}$ & $\begin{array}{l}5.9 \\
1.1 \\
7.0\end{array}$ \\
\hline \multicolumn{6}{|l|}{ No. 9 - West } \\
\hline $\begin{array}{l}\text { Electric } \\
\text { Gas } \\
\text { Total }\end{array}$ & $\begin{array}{r}17.9 \\
2.1 \\
20.0\end{array}$ & $\begin{array}{r}19.5 \\
2.2 \\
21.7\end{array}$ & $\begin{array}{r}21.2 \\
2.3 \\
23.5\end{array}$ & $\begin{array}{r}22.7 \\
2.5 \\
25.2\end{array}$ & $\begin{array}{r}25.3 \\
2.8 \\
28.1\end{array}$ \\
\hline \multicolumn{6}{|l|}{ No. 10 - Northwest } \\
\hline $\begin{array}{l}\text { Electric } \\
\text { Gas } \\
\text { Total }\end{array}$ & $\begin{array}{l}7.5 \\
0.8 \\
8.3\end{array}$ & $\begin{array}{l}8.2 \\
0.0 \\
9.1\end{array}$ & $\begin{array}{l}8.8 \\
1.0 \\
9.8\end{array}$ & $\begin{array}{r}9.5 \\
1.0 \\
10.5\end{array}$ & $\begin{array}{r}10.4 \\
1.2 \\
11.6\end{array}$ \\
\hline \multicolumn{6}{|l|}{ United States } \\
\hline $\begin{array}{l}\text { Electric } \\
\text { Gas } \\
\text { Total }\end{array}$ & $\begin{array}{r}145.1 \\
21.7 \\
166.8\end{array}$ & $\begin{array}{r}157.4 \\
23.5 \\
180.9\end{array}$ & $\begin{array}{r}170.6 \\
25.5 \\
196.1\end{array}$ & $\begin{array}{r}182.7 \\
27.3 \\
210.0\end{array}$ & $\begin{array}{r}202.5 \\
30.4 \\
233.9\end{array}$ \\
\hline
\end{tabular}

1. Total demand was based on additional commercial floor space and demands repre. sentative of individual bullding types, using data obtained from: Disaggreaation by Fuel, Bullding Type, and End Use, Oak RIdge National Laboratory, ORNL/CON-14, February 1978 .

(3) Volume Three, DOE/E1A-0173/3. (3) ASFRAE data

z. Uenand was allocated among and within regions using estimates of regional energy use derived from data in Energy Information Administration. Annual Report to Congress 1978, Volume Three, DoE/EIA-0173/3 and Brookhaven Research Institute, EPRI EA-462, June 1977. 


\section{EXHIBIT II-9 \\ New Hater Heating Demand in Commercial offices, Schools and Retail Stores (Gigawatts) 1}

\begin{tabular}{|c|c|c|c|c|c|}
\hline DOE REGION/FUEL TYPE & 1980 & 1985 & 1990 & 1995 & 2000 \\
\hline \multicolumn{6}{|l|}{ No. 1 - New England } \\
\hline $\begin{array}{l}\text { Electric } \\
\text { Gess } \\
\text { Oi1 } \\
\text { Totol }\end{array}$ & $\begin{aligned}<0.1 \\
<0.1 \\
0.7 \\
0.7\end{aligned}$ & $\begin{array}{l}<0.1 \\
0.1 \\
0.7 \\
0.8\end{array}$ & $\begin{array}{r}0.2 \\
<0.1 \\
0.7 \\
0.9\end{array}$ & $\begin{array}{r}<.2 \\
<0.1 \\
0.7 \\
0.9\end{array}$ & $\begin{array}{l}0.2 \\
0.1 \\
0.7 \\
1.0\end{array}$ \\
\hline \multicolumn{6}{|c|}{ No. 2 - New rork/New Jersey } \\
\hline $\begin{array}{l}\text { Electric } \\
\text { Gass } \\
\text { oil } \\
\text { Total }\end{array}$ & $\begin{array}{l}0.2 \\
.0 .5 \\
1.0 \\
1.7\end{array}$ & $\begin{array}{l}0.3 \\
0.5 \\
1.0 \\
1.8\end{array}$ & $\begin{array}{l}0.4 \\
0.5 \\
1.0 \\
1.9\end{array}$ & $\begin{array}{l}0.5 \\
0.5 \\
1.0 \\
2.0\end{array}$ & $\begin{array}{l}0.5 \\
0.5 \\
1.1 \\
2.2\end{array}$ \\
\hline \multicolumn{6}{|l|}{ No. 3 - Mid Atlantic } \\
\hline $\begin{array}{l}\text { Electric } \\
\text { Gas } \\
\text { O\$1 } \\
\text { Ootal }\end{array}$ & $\begin{array}{l}0.2 \\
0.6 \\
1.1 \\
1.8\end{array}$ & $\begin{array}{l}0.3 \\
0.6 \\
1.0 \\
1.9\end{array}$ & $\begin{array}{l}0.4 \\
0.6 \\
1.1 \\
2.1\end{array}$ & $\begin{array}{l}0.5 \\
0.6 \\
1.1 \\
2.2\end{array}$ & $\begin{array}{l}0.6 \\
0.8 \\
1.0 \\
2.4\end{array}$ \\
\hline \multicolumn{6}{|l|}{ No. 4 - South Atlantic } \\
\hline $\begin{array}{l}\text { Electric } \\
\text { Gas } \\
0 \text { i1 } \\
\text { Total }\end{array}$ & $\begin{array}{l}0.3 \\
1.0 \\
1.1 \\
2.4\end{array}$ & $\begin{array}{l}0.4 \\
1.0 \\
1.1 \\
2.5\end{array}$ & $\begin{array}{l}0.5 \\
1.2 \\
1.1 \\
2.8\end{array}$ & $\begin{array}{l}0.7 \\
1.0 \\
1.1 \\
2.9\end{array}$ & $\begin{array}{l}0.9 \\
1.2 \\
1.1 \\
3.2\end{array}$ \\
\hline \multicolumn{6}{|l|}{ No. 5 - Midwest } \\
\hline $\begin{array}{l}\text { Electric } \\
\text { Gass } \\
\text { of } \\
\text { Total }\end{array}$ & $\begin{array}{l}0.3 \\
1.3 \\
0.9 \\
2.5\end{array}$ & $\begin{array}{l}0.5 \\
1.4 \\
0.8 \\
2.7\end{array}$ & $\begin{array}{l}0.6 \\
0.4 \\
0.4 \\
2.8\end{array}$ & $\begin{array}{l}0.8 \\
1.5 \\
0.8 \\
3.1\end{array}$ & $\begin{array}{l}1.1 \\
1.5 \\
0.8 \\
3.4\end{array}$ \\
\hline \multicolumn{6}{|l|}{ No. 6 - Southeast } \\
\hline $\begin{array}{l}\text { Electric } \\
\text { Gas } \\
\text { Oil } \\
\text { Total }\end{array}$ & $\begin{array}{l}0.3 \\
0.9 \\
0.4 \\
1.6\end{array}$ & $\begin{array}{l}0.3 \\
0.9 \\
0.6 \\
1.8\end{array}$ & $\begin{array}{l}0.4 \\
0.9 \\
0.6 \\
1.9\end{array}$ & $\begin{array}{l}0.5 \\
0.9 \\
0.6 \\
2.0\end{array}$ & $\begin{array}{l}0.6 \\
1.0 \\
0.6 \\
2.2\end{array}$ \\
\hline
\end{tabular}

\section{H10. 7 -. Central}

Electric

Gas

Totel

$\begin{array}{lllll}0.1 & 0.2 & 0.2 & 0.3 & 0.4 \\ 0.5 & 0.5 & 0.6 & 0.6 & 0.6 \\ 0.4 & 0.4 & 0.2 & 0.4 & 0.4 \\ 1.0 & 1.1 & 1.2 & 1.3 & 1.4\end{array}$

No. 8 - North Central

Electric

Gas

Total

$\begin{array}{lllll}0.1 & 0.1 & 0.1 & 0.1 & 0.2 \\ 0.3 & 0.3 & 0.3 & 0.4 & 0.4 \\ 0.2 & 0.2 & 0.3 & 0.2 & 0.2 \\ 0.6 & 0.6 & 0.7 & 0.7 & 0.8\end{array}$

No. 9 - Mest

Electric

Gas
oil
Tota

$\begin{array}{lllll}0.2 & 0.2 & 0.4 & 0.5 & 0.6 \\ 1.0 & 1.0 & 1.0 & 1.0 & 1.0 \\ 0.6 & 0.6 & 0.5 & 0.5 & 0.5 \\ 1.8 & 1.8 & 1.9 & 2.0 & 2.1\end{array}$

No. 10 - Nortmest

Clectric

Gas

$\begin{array}{lllll}0.1 & 0.2 & 0.2 & 0.2 & 0.3 \\ 0.6 & 0.6 & 0.6 & 0.6 & 0.8 \\ 0.2 & 0.2 & 0.2 & 0.3 & 0.3 \\ 0.9 & 1.0 & 1.0 & 1.1 & 1.2\end{array}$

United States

\begin{tabular}{lrrrrr} 
Electric & & & & & \\
685 & 1.8 & 2.5 & 3.4 & 4.3 & 5.5 \\
011 & 6.7 & 6.9 & 7.1 & 7.2 & 7.9 \\
Tozal & 6.5 & 6.6 & 6.7 & 6.7 & 6.7 \\
\hline & 15.0 & 16.0 & 17.2 & 18.2 & 19.9 \\
\hline
\end{tabular}

1. Tozal densnd was based on edditional commercial floor space and denands

Fepresentative of individual building type. Us ing dats obtained from:

Butlaine Type, ane End Use, Oak Ridge

LAbordtory, ORNL/CON-14, rebruary 1978.

(2) Energy Information Admintstration. Annual Regart to Congress 1978

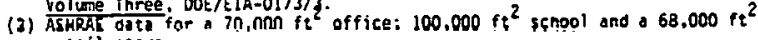

2. Demand was aliocated among ond within regions using estimazes of regional energy use derived from dato in Energy informbtion Adarinistration. Annual Report to Congress 1978, Volume Three. DOE/EIA-0173/3 and Brookhaven Poner Reseerch institute, EPRI EA-462. June 1977 
3. FROM THE PERSPECTIVE OF END-USE APPLICATIONS, TES IS MOST ECONOMICAI: COMPARED TO COMMERCIAI AIR CONDITIONING SYSTEMS AND RESIDENTIAI SPACE HEATING SYSTEMS

On an economic basis, a large potential exists for TES to displace commercial air conditioning and, to a lesser extent, residential space heating systems. As shown in Exhibit II-10, TES is economic with over 90 percent of conventional air conditioning demand, mainly commercial, and over 65 percent of residential space heating. These two end uses of TES represent about $116 \mathrm{GW}$ and $76 \mathrm{GW}$ respectively. While TES could displace nearly 50 percent of the residential/commercial water heating demand, the size of this market is relatively small, $22 \mathrm{GW}$.

Summaries of the residential and commercial demands for space heating, water heating and air conditioning demand are shown in Exhibits II-5 through 9, and details of the economics and energy use are presented in Appendices $B, C$, and $D$.

The economic market from the perspective of the end user, also is potentially large. Since the selection economics of TES and conventional alternatives are highly situation specify, the economic attractiveness of. TES was based on a regional life-cycle cost analysis.

Chapter III discusses the expected market for TES. 
EXHIBIT II-10

TES Market by End Use

$\stackrel{w}{w}$

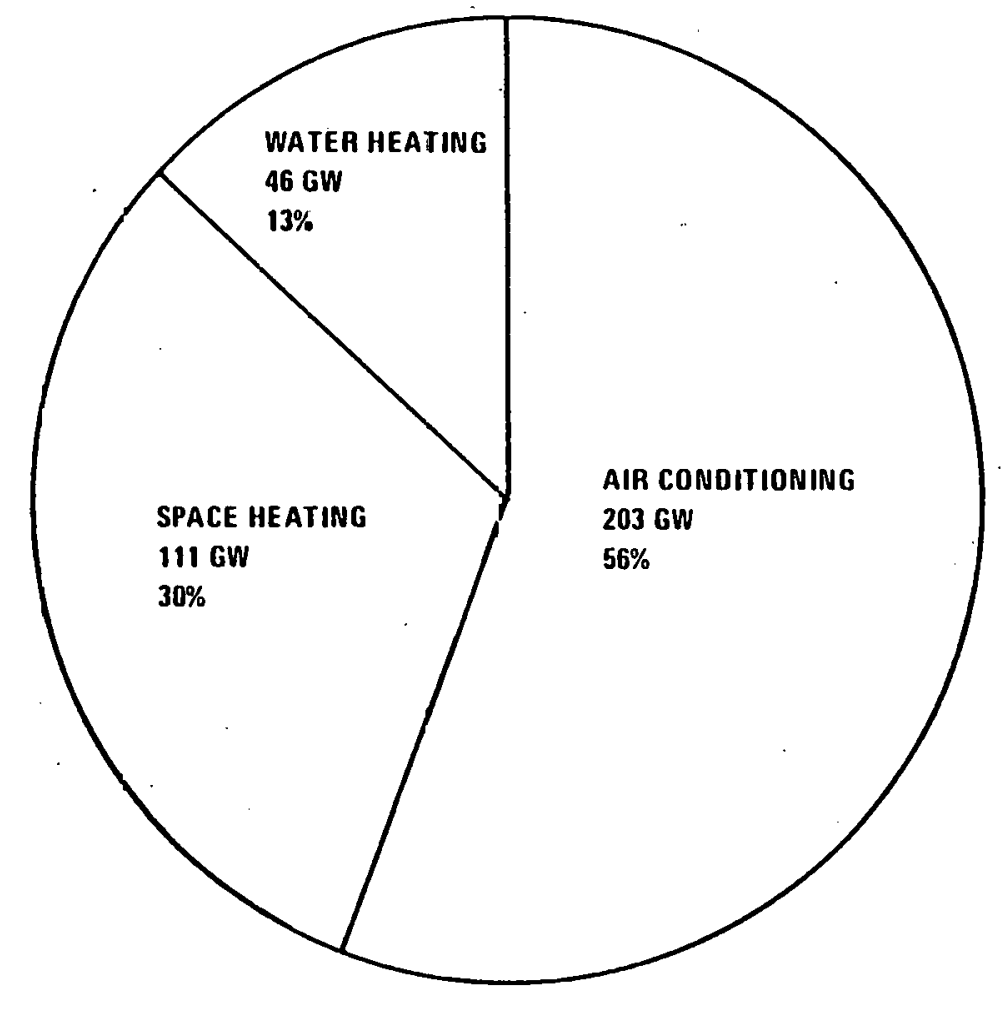

TOTAL RESIDENTIAL AND COMMERCIAL

DEMAND - 360 GW (1980)

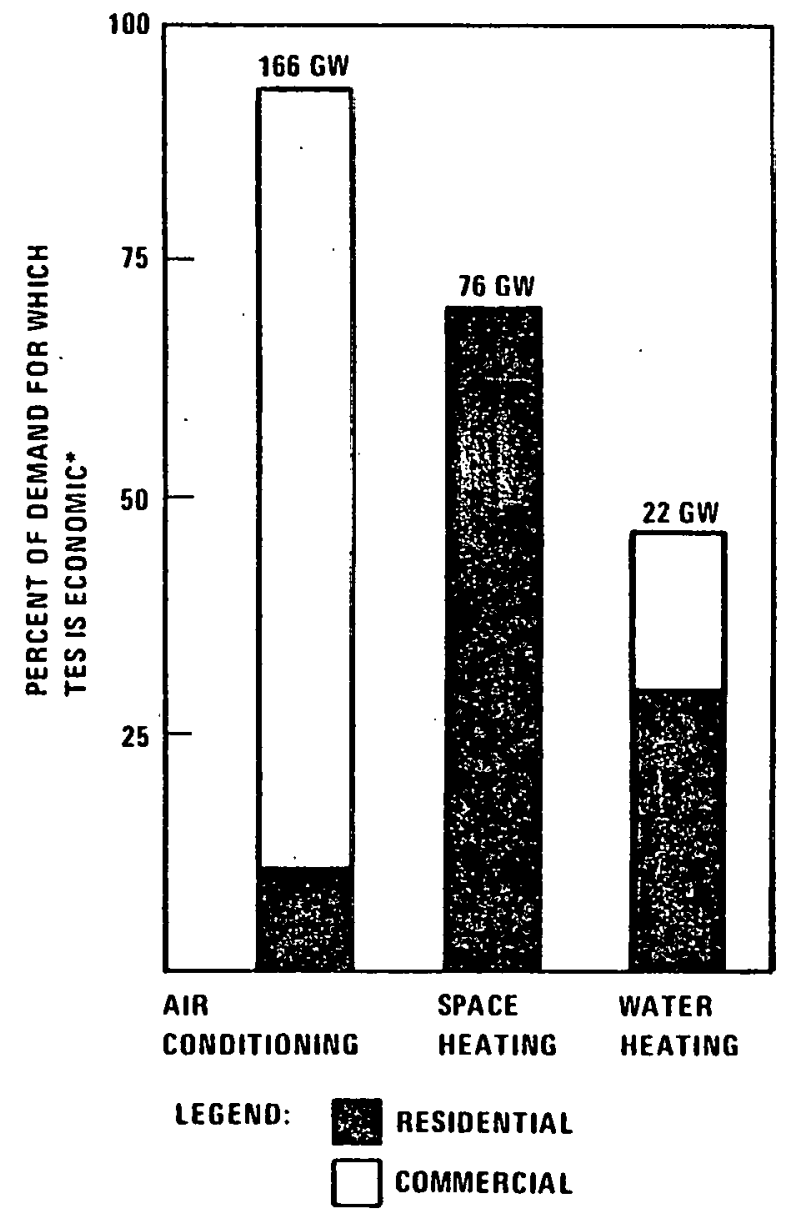

* based on comparative life-cycle costs 


\section{EXPECTED TES MARKET}

Penetration into the residential and commercial markets for space heating, water heating, and air conditioning is complicated by various factors -- both supply and demand related. These factors, many of which have been addressed in prior studies, 4,11 act to enhance or inhibit the development of a TES market. The relative importance of each factor is assimilated in judgments of the expected market penetration.

Presently, the use of TES is miniscule, limited to installations dispersed throughout the New England, Mid-Atlantic and Midwest regions. For example, Vermont's two largest utilities have 425 TES customers and the American Electric Power Company serves 70 TES customers. However, with the utilization of preferential utility rates and the development of aggressive public awareness programs, the TES market is likely to burgeon into a multi-gigawatt opportunity.

As shown in Exhibit III-1, TES could account for about 4 percent of the total residential and commercial demand for space heating, water heating and air conditioning. Commercial air conditioning and residential space heating would be the two largest markets for TES, consisting of 80 percent of potential demand. The remaining 20 percent is comprised of residential water heating and air conditioning, and commercial water heating. Generally, the need for commercial space heating affords much less of an opportunity for TES. 3,12

The expected market of these TES applications is developed from consideration of:

The attitudes and awareness of customers to preferential rate structures and other TES characteristics

- The expected market Fenetration

- The production capability of manufacturers

- The requirements for new instatallations. 
EXHIBIT III-1

Frojected TES Market By the Year 2000
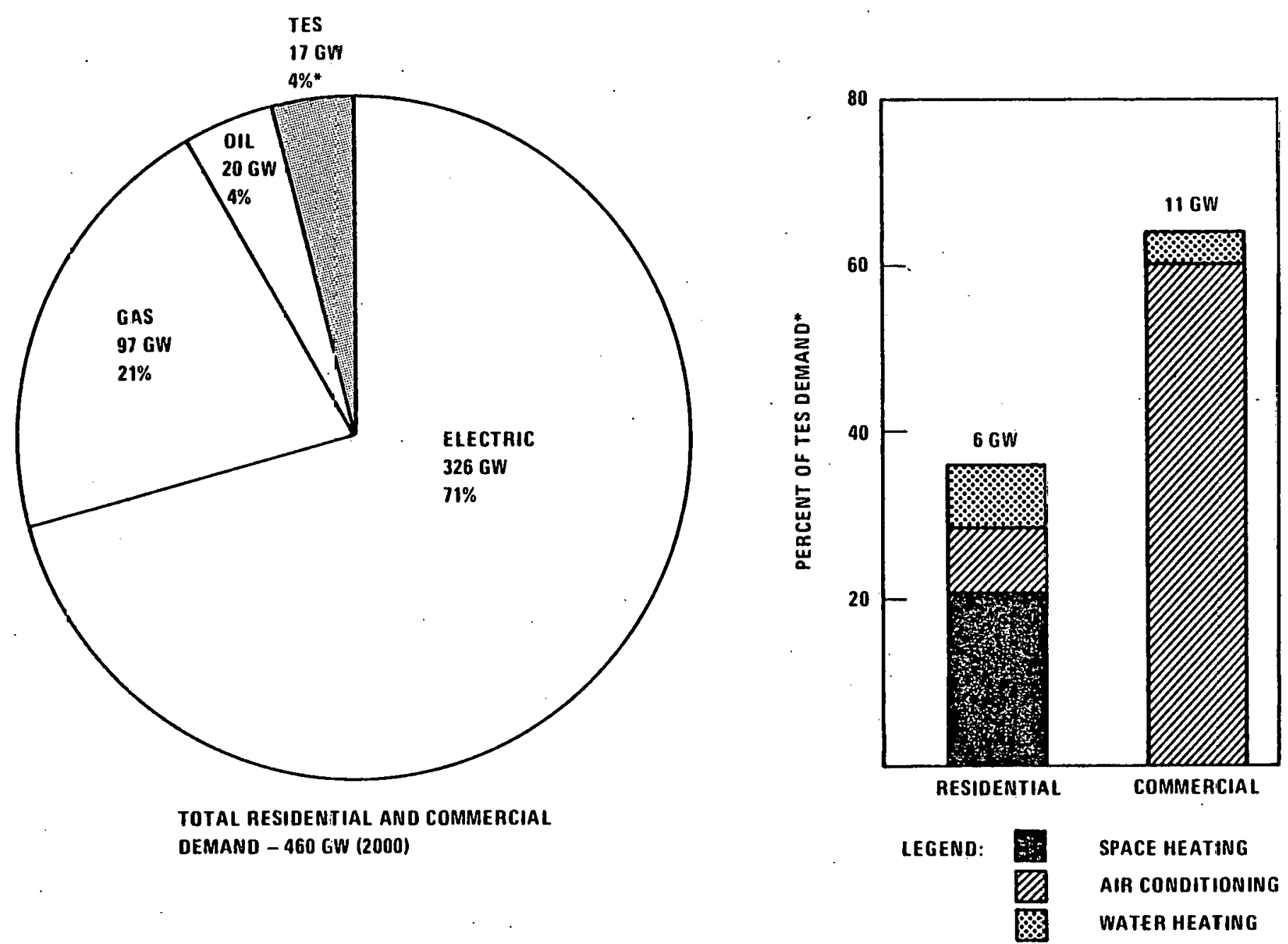

* ASSUMES TES WILL BE 5\% OF NEW INSTALLATIONS WITH WHICH IT IS ECONOMIC 
The remainder of this chapter discusses these factors in greater detail.

1. THE TES MARKET SHOULD INCREASE AS RATES FAVORABLE TO TES ARE ESTABIISHED AND PUBLIC AWARENESS INCREASES

In 1972 and 1973 the University of Pennsylvania conducted two market surveys for off-peak air conditioning. During the course of the study, their initial characterizations of the residential and. commercial customers as skeptical changed to one of "active interest and a desire to use novel, unconventional energy sources". 13 The reason for this change in attitude was the rising cost of energy precipitated by the oil crisis of 1973. As a result, customers today are more open-minded about attenative energy sources, if the opportunity is present. This section discusses:

- The current lack of public awareness of TES

- The conservatism of public utility commissions to anticipated customer unresponsiveness

- The acceptance among those familiar with. TES

- The expected favorable response to TES.

(1) Currently, the Public Generally is Uninformed About TES

A key to the development of the market for TES is awareness by builders, developers and customers. Experience in North Carolina has demonstrated that the knowledge of TES by builders/developers is crucial to the success of TES since they are the ones whose decision it is to install TES in new houstng units. 14 Discussions with home and commercial building associations across the country have indicated that awareness of TES among them is very low.

Except in a few locations, such as Vermont, North Carolina and Wisconsin, where there are TFS demonstrations, customers also are ignorant of TES. The situation can be best described by Richard de Grasse, Vice President of Control Electric Corporation, "Public awareness of storage hardware is almost non-existent except in markets where we have been active for several years". 15 
Diffusion of information about TES has been slow because the industry is highly fragmented and their technical staffs have been greatly reduced in recent years. For example, it is estimated that there are over 127,000 residential builders in the United States. of these, about 500 construct about 20 to 30 percent of the single family homes built each year. The sheer number of even the major companies slows the penetration of any innovation. In adition, the fluctuating housing market and fear of recession have resulted in builders' not supporting in-house technical staffs. This situation tends to slow. the transfer of information, because of the difficulty in accessing key decisionmakers in the building industry.

(2) The Current Conservatism of State Utility Commissions Towards TES is Indicative of a General Feeling That Customers Will be Unresponsive to New Rate Designs

Since they play a fundamental role in promoting and establishing rate designs, state public utility commissions significantly influence TES market development. The current overall attitude of state public utility commissions towards TES is best characterized as conservative. Although commissions in a number of states have expressed interest in TES on an experimental basis, they appear reluctant to take steps to further encourage TES until the results of pilot studies can be evaluated. However, commission staffs indicate that TES, together with other load management techniques, has been and will continue to be, considered at generic rate hearings.

State utility commissions generally, are concerned that customers will not accept new rate designs that are conducive to the develupment of TES. Their concerns primarily involve the following issues:

Time of day (TOD) pricing, which is important to the successful implementation of TES, is still viewed as experimental by most commissions.

Most of the commission staff personnel interviewed consider some form of price incentive to be essential to the market penetration of TES, and identify TOD rates as the most 
likely form of incentive. While commission staffs suggest that TOD rates were a necessary condition for TES development, they note that such rates are currently available to only a small number of customers, and that rates are still viewed as experimental.

The reluctance of state commissions and utilities to pursue TOD on a larger scale reflects an overall apprehension regarding the lack of proven success of TOD rates. Commission staff personnel voice three main concerns. First, they note that price differentials between peak and off-peak prices under current TOD experiments are not always sufficient to motivate consumers to tolerate the inconvenience of utilizing electricity primarily in off-peak hours. Secondly, they point to the fact that there is no guarantee that peak to off-peak ratios will remain sufficiently attractive over time. Finally, commission staff suggest that TOD rates may be unnecessary for some utilities which have excess peak capacity or inappropriate mixes of fuel or generating equipment. These and other concerns over the viability of TOD rates represent a significant obstacle to TES market penetration since until TOD rates are available on a more extensive basis for a larger number of customers, the potential for the technology will be reduced.

Alternative energy sources may reduce the demand or need for TES.

TES is generally viewed as one of a number of load management strategies. Where other strategies are available to shift or reduce peak loads more conveniently or at a lower cost, the market for TES will be reduced. (However, it must be noted that there is at present, no viable alternative strategy for air conditioning other than cool storage). According to a staff person of Vermont's Public Service Board, for example, the availability and use of wood as a supplemental energy source poses considerable competition for TES in that state. Woodburning is becoming increasingly popular in Vermont as 
well as other northern states, and as long as wood is available at costs competitive with off-peak electricity it could reduce considerable the market for TES.

Customers participation may be low because they do not perceive the benefits of TES.

According to staff persons interviewed, the economic benefits of shifting to TES must be very apparent to the consumer, and furthermore, the potential user must be relatively sure that those benefits will continue cver time. In other words, the short- and longterm differentials in price between peak and off-peak prices must be sufficient to justify the initial investment in the units. Due to the great number of variables that affect rates over time, clearly it is very difficult to predict those long-term differentials.

This perception on the part of state commissions that consumer demand may be limited is significant, since commissions are less likely to actively encourage TFS if it appears that consumer participation may be minimal.

Public Acceptance is Generally High Among Potential Builders, Developers and Customers Who Are Aware Of TES

Builders and developers can use TES as a selling tool, because TES potentially saves the customer money. Reflecting the risk of installing an unmarketable product, builders/developers are very sensitive to the idiosyncrasies and attitudes of their customers. Recent market studies in the solar industry have shown that reduction of utility bills ranked first or second among the most important factors relatirg to the possible purchase of a solar energy system. 16 The opportunity for TES to save the customer money on his utility bills has been reported. 17 Experience with TES in the American Electric Power Company (AEP) service indicates that customers can save $\$ 150-\$ 500$ on the annual utility bills. 18 Builders/developers could take advantage of this selling point. 
For example, 69 out of 70 TES demonstration homes on the AEP system were sold with complete acceptance by both builders and customers.. As a result of a survey, AEP reports that customers experienced overall satisfaction with TES with respect to:

Lower electric bills

Recovery of initial investment (3-5 years) Comfort Reliability.

These systems included both electric storage space and water heating. Similar results have been reported in vermont. There, "besides being economical, customers reported that storage heating provided a more even and more comfortable heat than direct, baseboard electric heating". I9

(4) Therefore, Aggressive Public Awareness Programs Are Designed to Promote and Enhance the Commercialization of TES

The development of a domestic TES market ultimately rests on the complex behavior of the customer. His awareness of the benefits derived from TES is crucial to the success of TES. Through their Thermal Energy storage Program, the DOE is actively involved in developing and helping to commercializing thermal storage technology. TES in part of this program, which has a budget of $\$ 8.2$ million for FY1979.20 In addition, EPRI is continuing to study TES on a electric load management technology and electric utility rate designs. A comprehensive summary of activities in $R D \& D$ of low temperature TES techniques has recently been published by SERI. ${ }^{21}$ As a result of these publicly-sponsored activities and private advertisements, (as appeared in the November 27, 1979 issue of Newsweek) public awareness of TES should improve with concomitant success in TES market development.

2. THE PRIVATE SECTOR EXPECTS THAT THE TES MARKET WILL CONSTITUTE ABOUT FIVE PERCENT OF NEW INSTAILATIONS

An estimate of the market penetration of TES was based on the expert judgment of representatives of the private sector -- utilities, manufacturers and builders/developers. *

* The development of a methodology to develop market penetrations and a detailed market survey to gather empirically-based estimates of market penetration was beyond the scope of this study. 
The market penetration was a parameter that accounted for the psychology of the buyer and prospective customers' expected response to TES. This parameter embodied the relative importance of such decisionmaking variables as:

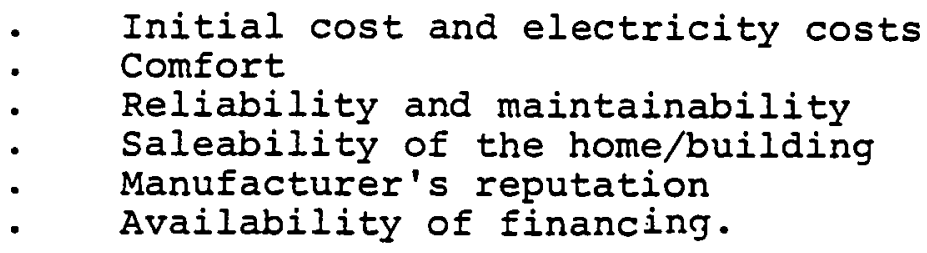

Based on information obtained from the private sector, it was estimated that about 5 percent of new installations with which TES is economical could be TES. Estimates generally were about 5 to 15 percent, although they varied widely. A 5 percent figure is consistent with published penetration estimates for TES associated with the use of solar energy systems in the residential and community sectors.12 Detailed buyer behavior studies in the solar energy field also have reportad unsubsidized market penetrations of about I - 3 percent of single family rental space, 4 - 9 percent for multi-family rental space and 3 percent for mid-rise office buildings and attached commercial stores. 22 The consensus of respondents in the solar study was that a five percent market penetration constituted a mature market.

While the European situation is not totally similar to the domestic market, the historical development of TES in Europe provides a useful comparison. For example, in Great Britain, there were no storage space heating units in 1952, and in 1956 there were less than 50,000 units of installed TES. By 1978, TES accounted for over 2.3 million units, or 12 percent of British households. In Germany, electric storage heating was introduced 15 years ago, and today TES is installed in about eight percent of German homes. 23

\section{MANUFACTURERS HAVE INDICATED THAT THEY WILL EXPAND PRODUCTION AS THE TES MARKET DEVELOPS}

Manufacturing firms planning to produce and sell TES hardware for the most part, expect a three to five year 
transition period between today's limited TES market and the need for full scale production. If, in fact, a large market developed sooner, domestic manufacturers would be unable to meet near-term demand. To a certain extent (in the stcrage heating market.), European manufacturers could help to make up this shortfall. The following sections describe briefly the manufacturing perspective on TES. A more comprehensive study of the industry assessment of energy storage is contemplated by DOE.

(1) The Developing TES Industry is Composed Primarily of Firms producing Related Heating and Cooling Systems

The firms considering the large scale development and marketing of distributed TES systems are for the most part, currently engaged in the manufacture and supply of space heating, cooling, and hot water systems and related electrical components. TES systems development, marketing, and distribution is therefore a logical extension of their current business activities. Exhibit III-2 presents a summary of the firms surveyed in developing the manufacturer's perspective on potential TES development in the United States. The exhibit indicates each firm's current business base, TES technology of interest, and stage of development.

The domestic TES market had its origins in the recognition by certain utilities (in the early 1970s) of the need for load management practices. Seeking various solutions, including alternative technologies as seen in Europe, the utilities approached the manufacturers of conventional heating and cooling systems. These firms therefore were among the first to become aware of potential TES applications and market possibilities for existing product lines. For example, A.O. Smith Corporation, a mid-west manufacturer of hot water heaters, was approached by Wisconsin Electric Power in 1974, to develop load management technology for ice bank storage and hot water storage systems. Subsequently, A.O. Smith built over 200 ice storage tanks for utility testing purposes, and two sizes (nine tons and thirteen tons air conditioning storage) are currently being marketed. Also, the company is producing the AEP load Leveling Storage Water Heater, which has been on the market now for over a year. 
EXHIBIT III-2

Manufacturers Surveyed/Industry Status

\begin{tabular}{|c|c|c|c|c|}
\hline & Company & Business Base & TES Technology & Stage of Development \\
\hline \multirow[t]{2}{*}{ Company } & A & $\begin{array}{l}\text { Hot Water Tanks } \\
\text { and Heaters (R/C) }\end{array}$ & $\begin{array}{l}\text { Storage Cooling (R) } \\
\text { (Ice) }\end{array}$ & $\begin{array}{l}\text { Released from Engineering to } \\
\text { Marketing }\end{array}$ \\
\hline & & & Storage Water Heaters (R) & On the Market 1 Year \\
\hline \multirow[t]{2}{*}{ Company } & B & Warm Fir Furnaces (C) & $\begin{array}{l}\text { Storage Heating (R) } \\
\text { (Phase Change Salts) }\end{array}$ & Demonstration/Testing \\
\hline & & & $\begin{array}{l}\text { storage Cooling (C) } \\
\text { (Phase Change Salts) }\end{array}$ & Initial Marketing \\
\hline Company & C & $\begin{array}{l}\text { Space Heating and } \\
\text { Air Conditioning (R/C) }\end{array}$ & $\begin{array}{l}\text { Storage Cooling }(\mathrm{R} / \mathrm{C}) \\
\text { (Ice) }\end{array}$ & $\begin{array}{l}\text { Utility Demonstration, } \\
\text { Limited Market Foreseen in } \\
\text { Residential Sector }\end{array}$ \\
\hline Company & $\mathbf{D}$ & $\begin{array}{l}\text { Electric storage Heating } \\
\text { (R) }\end{array}$ & $\begin{array}{l}\text { Storage Heating (R) } \\
\text { (Ceramic Brick) }\end{array}$ & $\begin{array}{l}4 \text { Years Active Sales, } \\
\text { Solid Market Base }\end{array}$ \\
\hline Company & $\mathbf{E}$ & $\begin{array}{l}\text { Electric Heating Systems } \\
\text { and components (R/C) }\end{array}$ & $\begin{array}{l}\text { Storage Heating (R/C) } \\
\text { (Ceramic Brick) }\end{array}$ & $\begin{array}{l}\text { Exploratory R\&D, No Active } \\
\text { Development }\end{array}$ \\
\hline Company & $\mathbf{F}$ & HVAC Applications (C) & $\begin{array}{l}\text { Storage Cooling (C) } \\
\text { (Ice) }\end{array}$ & $\begin{array}{l}30 \text { Years of Sales } \\
\text { (Accepted Standard for Dairy } \\
\text { Plants) }\end{array}$ \\
\hline \multirow[t]{2}{*}{ Company } & G & $\begin{array}{l}\text { Space Heating and } \\
\text { Hot Water (C) }\end{array}$ & $\begin{array}{l}\text { Storage Heating (C) } \\
\text { (water) }\end{array}$ & $\begin{array}{l}\text { Active Marketing, } \\
\text { Solid Market Base }\end{array}$ \\
\hline & & & $\begin{array}{l}\text { Storage Heating ( } \mathrm{R}) \\
\text { (Water) }\end{array}$ & $\begin{array}{l}\text { Exploratory, No Active } \\
\text { Marketing }\end{array}$ \\
\hline Company & $\mathbf{H}$ & $\begin{array}{l}\text { Electric Heating } \\
\text { Systems }(R / C)\end{array}$ & $\begin{array}{l}\text { Storage Heating (R) } \\
\text { (Ceramic Brick) }\end{array}$ & $\begin{array}{l}\text { Active Marketing, } \\
\text { Limited Demand }\end{array}$ \\
\hline
\end{tabular}

Legend: $(R)=$ Residential

(C) = Commercial 
As another example, companies such as Girton Manufacturing have been making commercial chillers with ice stcrage for over thirty years, as this is the accepted industry standard for cooling milk products in dairy plants. Once again, it is a simple extension of technology for the manufacturers of electric chillers to convert the units to ice storage by chilling liquids in storage during non-use periods. These are important considerations in determining the readiness and capability of firms to meet potentially high domestic de-mand for TES.

(2) Manufacturers Expect Modest Growth in the TES Market in the Near Term

With few exceptions, current demand for TES technology and components ranges from very limited to nonexistent. However, the firms interested in TES systems development expressed confidence that potential markets exist of sufficient size and duration to ensure the profitability of the venture. For the most part, these firms expect very limited market development over the next several years, followed by a transition period of three to five years in which TES interest becomes widespread, and full-scale production lines (e.g., 5,000 units/year) can be set up.

These expectations of market development are based on the commercialization barriers currently perceived by the TES manufacturers, and include:

- The lack of appropriate utility rate structures

The unfamiliarity of customers, contractors, and utilities with TES system applications and time of use rates.

Despite these barriers, however, local markets have developed where active marketing programs and government/utility demonstration programs are ongoing. For example, a New England storage heating unit distributor (whose sales have doubled each year for the past four years) states that TES market development is ensured following rate structure changes, but that it takes several years to get the market started even with favorable time of use rates and support from the utility and DOE. Thus, while manufacturers anticipate that widespread electric rate structure development 
will promote acceptance of the appropriate TES technologies, they are uncertain as to the rate of acceptance. These factors account for their expectation of modest market development in the next several years, and a transition period of three to five years to widespread acceptance of TES.

\section{(3) Anticipated Plans to Expand Production Should Meet TES Demand}

Initial manufacturing activity has been limited to utility and government sponsored projects (with certain exceptions; e.g., sales of room-size ceramic brick storage heaters in the New England region). However, growth of the industry is anticipated, and numerous firms are ready to grow with demand. For example, a manufacturer of 30-kw ceramic brick storage residential central heating systems is currently producing less than 100 units per year, but anticipates setting up an entire manufacturing facility with a production capability eventually exceeding 10,000 units per year, as demand increases over the next three to five years. The firm is planning to set up dealers (and bypass the distributors) to interface directly with the builders.

As another example, an electric components manufacturer with nationwide distributor outlets is actively exploring the market opportunity for ceramic brick storage heating systems, and could easily produce and distribute large quantities of these units as the market develops. This company hopes to be in this position in about five years.

Two manufacturers of ice storage units have plans within the next three to five years to build a 5,000 unit/year production line for commercial cool storage systems. Neither expects a residential cool storage market to develop except in limited instances where forced by the utility.

Thus, not only are the manufacturers confident of providing sufficient quantities of storage hardware to meet anticipated growth, they are also prepared, in most cases, with a supporting infrastructure (i.e., distributors and dealers) capable of meeting projected demand. However, should the demand be underestimated and rapid market penctration take place in the next 
two years, domestic manufacturers would be unable to keep up. In this situation, foreign suppliers would be in a position to make up the shortfall for certain technologies, primarily ceramic brick storage. This is true because, following the introduction of reduced off-peak rates in the early 1960's, the German TES market experienced growth rates of 50 percent per year for more than a decade. In this period, more than five million kilowatts of electric storage heating were installed,24 and the upward trend is continuing.

The European TES activity has resulted in a large inplace manufacturing capability, and several U.S. firms are licensed to sell and/or manufacture the European storage hardware. For example, nearly 4,000 Germanmade room-size ceramic brick storage heaters (mostly of the two kW size) will be sold in the New England region in 1979. Thus, foreign manufacturers are indeed capable of supplying certain types of TES technology in the early years of market development, and in fact are serving to demonstrate the potential benefits of TES systems to a growing number of people.

4. A MATURE TES MARKET COULD BE NEARLY $165 \mathrm{GW}$ (CUMULATIVE) BY THE YEAR 2000.

If TES were installed in 5 percent of the housing units for which it is economical, the TES market could be a multigigawatt business. Exhibit III-3 presents the estimated residential and commercial markets for TES for each percent of the total installations with which TES is economical on a life-cycle cost basis. This section describes the size of the potential market and its ability to be supported by off-peak utility capacity.

(1) The Residential Market for TES is Dominated by storage Space Heating and Could be Large

On a national level, the annual demand for TES capacity could be nearly $800 \mathrm{MW}$ for each percent of the total installation with which it is economical in 1980 and exceed 1,000 MW in the year 2000. (See Exhibit III-3). Most of this demand results from meeting space heating requirements. Both residential water heating and air conditioning account for less than half the demand for space heating. 
EXHIBIT III-3

Annual Market for Residential and Commercial TES

(Megawatts)

\begin{tabular}{|c|c|c|c|c|c|}
\hline DOE REGIUR/SECTOR/ENO USE & \multicolumn{5}{|c|}{ 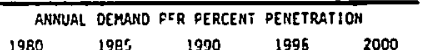 } \\
\hline $0.1 \cdot$. England & & & & & \\
\hline 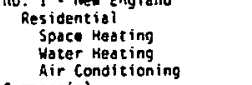 & $\begin{array}{l}39.0 \\
10.1 \\
0.1\end{array}$ & $\begin{array}{l}44.8 \\
11.1\end{array}$ & $\begin{array}{l}96.8 \\
11.7 \\
0\end{array}$ & $\begin{array}{l}42.0 \\
33: 0 \\
0\end{array}$ & $\begin{array}{l}37.4 \\
13.8 \\
0\end{array}$ \\
\hline 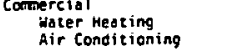 & $\begin{aligned} 72.0 \\
29.0\end{aligned}$ & $\begin{array}{l}3.0 \\
31.0\end{array}$ & $\begin{array}{l}9.0 \\
33.0\end{array}$ & 39.0 & $\begin{array}{l}9.0 \\
39.0\end{array}$ \\
\hline 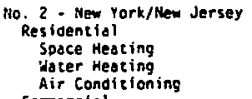 & $\begin{array}{l}4.8 \\
8.1 \\
0\end{array}$ & $\begin{array}{l}5.7 \\
9.0 \\
0\end{array}$ & $\begin{array}{l}\frac{6}{60.0} \\
10.6 \\
0\end{array}$ & $\begin{array}{l}3.3 \\
12.8 \\
0\end{array}$ & $\begin{array}{l}11.9 \\
15.4 \\
0\end{array}$ \\
\hline 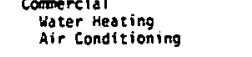 & $\begin{array}{l}12.0 \\
57.0\end{array}$ & $\begin{array}{l}13.0 \\
62.0\end{array}$ & $\begin{array}{l}14.0 \\
66.0\end{array}$ & $\begin{array}{l}15.0 \\
11.0\end{array}$ & $\begin{array}{l}17.0 \\
80.0\end{array}$ \\
\hline 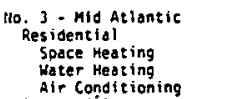 & $\begin{array}{l}\frac{60.7}{16.8} \\
46.5\end{array}$ & $\begin{array}{l}65.1 \\
18.1 \\
66.3\end{array}$ & $\begin{array}{l}57.6 \\
19.5 \\
69.3\end{array}$ & $\begin{array}{l}56.5 \\
20.2 \\
51.1\end{array}$ & $\begin{array}{l}66.8 \\
20.8 \\
52.4\end{array}$ \\
\hline 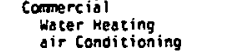 & $\begin{array}{l}13.0 \\
212.0\end{array}$ & $\begin{array}{l}1330.0 \\
230.0\end{array}$ & $\begin{array}{r}15.0 \\
250.0\end{array}$ & $\begin{array}{l}166.0 \\
265.8\end{array}$ & $\begin{array}{l}16.0 \\
298.0\end{array}$ \\
\hline 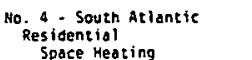 & & & & & \\
\hline 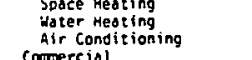 & $\begin{array}{l}1533.9 \\
39.9 \\
81.2\end{array}$ & $\begin{array}{l}106.2 \\
41.5 \\
86.1\end{array}$ & $\begin{array}{l}172.9 \\
43.7 \\
92.5\end{array}$ & 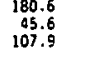 & $\begin{array}{l}188.5 \\
\text { s4.5. } \\
\text { 103.2 }\end{array}$ \\
\hline 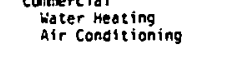 & $\begin{array}{l}14.0 \\
297.0\end{array}$ & $\begin{array}{l}15.0 \\
323.0\end{array}$ & $\begin{array}{l}16.0 \\
351.0\end{array}$ & $\begin{array}{l}38.0 \\
375.0\end{array}$ & $\begin{array}{r}20.0 \\
016.0\end{array}$ \\
\hline 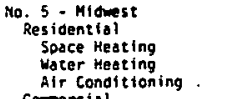 & $\begin{array}{l}73.6 \\
22.1 \\
2 \\
0.0\end{array}$ & $\begin{array}{l}\frac{88.6}{28.9} \\
2 \\
0\end{array}$ & $\begin{array}{l}98.4 \\
32.7 \\
0\end{array}$ & $\begin{array}{l}\frac{111.5}{38.2} \\
0.2\end{array}$ & $\begin{array}{l}131.2 \\
34 . .8\end{array}$ \\
\hline 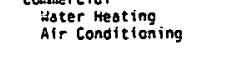 & $\begin{array}{l}169.0 \\
169.0\end{array}$ & $\begin{array}{l}138.0 \\
18.0\end{array}$ & $\begin{aligned} 14.0 \\
200.0\end{aligned}$ & $\begin{array}{l}26.0 \\
214.0\end{array}$ & $\begin{array}{l}299.0 \\
239.0\end{array}$ \\
\hline 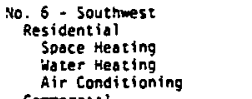 & $\begin{array}{l}\text { s5.1. } \\
13.9 \\
68.1\end{array}$ & $\begin{array}{l}58.2 \\
22.1 \\
71.5\end{array}$ & $\begin{array}{l}82.6 \\
29.7 \\
75.6\end{array}$ & 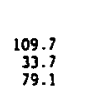 & $\begin{array}{r}333.9 \\
335.9 \\
82.7\end{array}$ \\
\hline 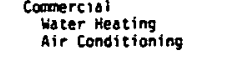 & $\begin{aligned} 91.0 \\
345.0\end{aligned}$ & $\begin{array}{l}9.0 \\
373.0\end{array}$ & $\begin{aligned} 100.0 \\
405.0\end{aligned}$ & $\begin{array}{l}111.0 \\
434.0\end{array}$ & $\begin{array}{l}12.0 \\
483.0\end{array}$ \\
\hline 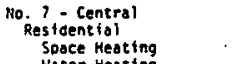 & 12.0 & 14.0 & 17.5 & 23.9 & \\
\hline 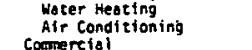 & $\begin{array}{c}52.5 \\
22.3\end{array}$ & $\begin{array}{l}73.0 \\
23.1\end{array}$ & $\begin{array}{l}9.7 \\
23.3\end{array}$ & $\begin{array}{l}12.4 \\
23.3\end{array}$ & $\begin{array}{l}14.6 \\
23.4\end{array}$ \\
\hline 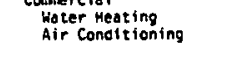 & $\begin{array}{l}5.0 \\
45.0\end{array}$ & $\begin{array}{l}6.0 \\
48.0\end{array}$ & $\begin{array}{l}6.0 \\
51.0\end{array}$ & $\begin{array}{ll}76.0 \\
s c .0\end{array}$ & $\begin{array}{l}8.0 \\
60.0\end{array}$ \\
\hline 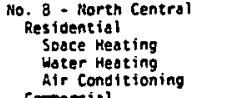 & $\begin{array}{l}8.3 \\
8.6 \\
0.6\end{array}$ & $\begin{array}{l}13.7 \\
6.2 \\
6.2\end{array}$ & $\begin{array}{l}16.8 \\
7.9 \\
0\end{array}$ & $\begin{array}{l}22.2 \\
9.8 \\
0\end{array}$ & $\begin{array}{l}29.9 \\
11.6 \\
0\end{array}$ \\
\hline 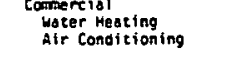 & $\begin{array}{l}3.0 \\
63.0\end{array}$ & $\begin{array}{l}3.0 \\
46.0\end{array}$ & $\begin{array}{l}s .0 \\
50.0\end{array}$ & $\begin{array}{l}3.0 \\
54.0\end{array}$ & $\begin{array}{l}49.0 \\
59.0\end{array}$ \\
\hline 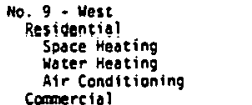 & $\begin{array}{l}23.4 \\
13.4 \\
0.4\end{array}$ & $\begin{array}{l}4.46 .1 \\
33.6 \\
0\end{array}$ & $\begin{array}{l}94.8 \\
39.6 \\
0.6\end{array}$ & 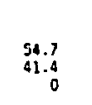 & $\begin{array}{l}52.08 \\
62.8 \\
0 \\
0.8\end{array}$ \\
\hline 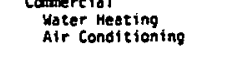 & $\begin{array}{l}88.0 \\
178.0\end{array}$ & $\begin{array}{l}88.0 \\
195.0\end{array}$ & 212.0 & 227.0 & $\begin{array}{l}212.0 \\
255: 0\end{array}$ \\
\hline 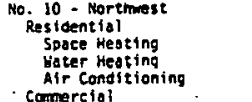 & $\begin{array}{r}24.5 \\
0 \\
0 .\end{array}$ & $\begin{array}{r}25.2 \\
0 \\
0\end{array}$ & $\begin{aligned} 26.3 \\
0 \\
0\end{aligned}$ & $\begin{array}{r}26.5 \\
0 \\
0\end{array}$ & $\begin{aligned} 26.5 \\
0 \\
0\end{aligned}$ \\
\hline $\begin{array}{l}\text { Kacter Meating } \\
\text { Ait Conotit ioning }\end{array}$ & $\begin{array}{l}33.0 \\
75.0\end{array}$ & $\begin{array}{l}48.0 \\
B 2.0\end{array}$ & 88.0 & $\begin{array}{l}55.0 \\
95.0\end{array}$ & $\begin{array}{r}604.0 \\
104.0\end{array}$ \\
\hline 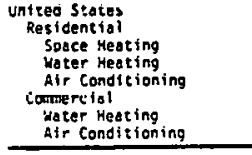 & 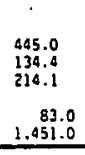 & $\begin{array}{l}524.0 \\
177.3 \\
227.0 \\
27.0 \\
1.911 .0 \\
1.574 .0 \\
\end{array}$ & $\begin{array}{l}589.7 \\
282.9 \\
20.7 \\
1.091 .0 \\
1.7050 .0 \\
\end{array}$ & $\begin{array}{r}625.99 \\
221.9 \\
261.4 \\
1.10 .0 \\
1.221 .0 \\
\end{array}$ & $\begin{array}{l}715.1 \\
26.1 \\
261.8 \\
2.122 .0 \\
2.0355 .0 \\
2\end{array}$ \\
\hline
\end{tabular}


Regionally, the largest demand for space heating occurs in the Mid-Atlantic, Midwest, South Atlantic and Southwest regions. The high demand in the southern region is indicative of the forecast high growth, and consequently, new housing units. The large space heating requirements of the Northeast, central and Northwest are moderated by the expected lower growth. As expected, the air conditioning loads are concentrated in the south Atlantic and southwest regions. Water heating loads generally are evenly distributed among the 10 regions.

The Commercial Market for TES Which is Principally Air Conditioning, Could Exceed the Ievel of the Residential Market

The commercial market for TES air conditioning could be over $1.5 \mathrm{GW}$ for each percent of new installations. The largest demand is in the Mid-Atlantic, South Atlantic, Southwest and West.

The commercial water heating market remains small in relation to other opportunities throughout the twenty-year period.

\section{Available Off-Peak Utility Capacity Should be Adequate to Support TES in Most Regions of the United States}

The TES market cannot exceed a level that could be supported by available off-peak utility capacity. The cumulative effect of annual installations of TES load per percent penetration is shown in Exhibit III-4.

Exhibits III-5 and III-6 show the cumulative effect of instaliing new TES capacity in the utility system on a national and regional basis, respectively. Excepting those regions where there was a very large commercial air conditioning load, most utilities should be able to accommodate a 5 percent penetration of TES. However, in those regions where the utility capacity places an upper bound on penetration, TES installations would have to proceed incrementally and be limited.

These estimates were based on the assumption that the utilities could allow 50 percent alversity, i.e., two eight hour charging periods over a twenty-four. 


\section{EXHIBIT III 4 \\ Cumulative Demand for Residential \\ and Commercial TES \\ (Gigawatts)}

\begin{tabular}{|c|c|c|c|c|c|}
\hline \multirow[b]{2}{*}{ DOE REGION/END USE } & \multicolumn{5}{|c|}{ Cumulative Demand Per Percent Penetration } \\
\hline & 1980 & 1985 & 1990 & 1995 & 2000 \\
\hline \multicolumn{6}{|l|}{ No. 1--New England } \\
\hline $\begin{array}{l}\text { Space \& Water Heat } \\
\text { Air Conditioning \& Water Heat }\end{array}$ & $\dot{-}$ & $\begin{array}{l}0.16 \\
0.13\end{array}$ & $\begin{array}{l}0.34 \\
0.26\end{array}$ & $\begin{array}{l}0.51 \\
0.40\end{array}$ & $\begin{array}{l}0.68 \\
0.56\end{array}$ \\
\hline \multicolumn{6}{|l|}{ No. 2--New York/New Jersey } \\
\hline $\begin{array}{l}\text { Space \& Water Heat } \\
\text { Air Conditioning \& Water Heat }\end{array}$ & - & $\begin{array}{l}0.06 \\
0.22\end{array}$ & $\begin{array}{l}0.12 \\
0.46\end{array}$ & $\begin{array}{l}0.20 \\
0.72\end{array}$ & $\begin{array}{l}0.29 \\
1.00\end{array}$ \\
\hline \multicolumn{6}{|l|}{ No. 3--Mid Atlantic } \\
\hline $\begin{array}{l}\text { Space \& water Heat } \\
\text { Air Conditioning \& Water Heat }\end{array}$ & $\because$ & $\begin{array}{l}0.25 \\
0.85\end{array}$ & $\begin{array}{l}0.51 \\
1.79\end{array}$ & $\begin{array}{l}0.79 \\
2.78\end{array}$ & $\begin{array}{l}1.06 \\
3.85\end{array}$ \\
\hline \multicolumn{6}{|l|}{ No. 4--South Atlantic } \\
\hline $\begin{array}{l}\text { Space \& Water Heat } \\
\text { Air Conditioning \& Water Heat }\end{array}$ & $\overline{-}$ & $\begin{array}{l}0.59 \\
1.29\end{array}$ & $\begin{array}{l}1.21 \\
2.69\end{array}$ & $\begin{array}{l}1.86 \\
4.20\end{array}$ & $\begin{array}{l}2.55 \\
5.83\end{array}$ \\
\hline \multicolumn{6}{|l|}{ No. 5--Midwest } \\
\hline $\begin{array}{l}\text { Space \& Water Heat } \\
\text { Air Conditioning \& Water Heat }\end{array}$ & - & $\begin{array}{l}0.32 \\
0.61\end{array}$ & $\begin{array}{l}0.69 \\
1.27\end{array}$ & 1.10 & $\begin{array}{l}1.58 \\
2.79\end{array}$ \\
\hline No. 6--Southwest & & & & & \\
\hline $\begin{array}{l}\text { Space \& Water Heat } \\
\text { Air Conditioning \& Water Heat }\end{array}$ & $\overline{-}$ & $\begin{array}{l}0.21 \\
1.34\end{array}$ & $\begin{array}{l}0.49 \\
2.80\end{array}$ & $\begin{array}{l}0.86 \\
4.37\end{array}$ & $\begin{array}{l}1.32 \\
6.08\end{array}$ \\
\hline \multicolumn{6}{|l|}{ No. 7--Central } \\
\hline $\begin{array}{l}\text { Space \& Water Heat } \\
\text { Air Conditioning \& Water Heat }\end{array}$ & $\dot{-}$ & $\begin{array}{l}0.06 \\
0.23\end{array}$ & $\begin{array}{l}0.14 \\
0.48\end{array}$ & $\begin{array}{l}0.24 \\
0.74\end{array}$ & $\begin{array}{l}0.33 \\
1.02\end{array}$ \\
\hline No. 8--North Central & & $\cdot$ & & & \\
\hline $\begin{array}{l}\text { Space \& Water Heat } \\
\text { Air Conditioning \& Water Heat }\end{array}$ & - & $\begin{array}{l}0.05 \\
0.15\end{array}$ & $\begin{array}{l}0.12 \\
0.32\end{array}$ & $\begin{array}{l}0.20 \\
0.50\end{array}$ & $\begin{array}{l}0.31 \\
0.69\end{array}$ \\
\hline No. 9--West & & & & & \\
\hline $\begin{array}{l}\text { Space \& Water Heat } \\
\text { Air Conditioning \& Water Heat }\end{array}$ & $\dot{r}$ & $\begin{array}{l}0.16 \\
0.62\end{array}$ & $\begin{array}{l}n .4 n \\
i .32\end{array}$ & $\begin{array}{l}0.67 \\
2.08\end{array}$ & $\begin{array}{l}0.93 \\
2.91\end{array}$ \\
\hline \multicolumn{6}{|l|}{ No. 10--Northwest } \\
\hline $\begin{array}{l}\text { Space \& Water Heat } \\
\text { Air Conditioning \& Water Heat }\end{array}$ & $\dot{-}$ & $\begin{array}{l}0.08 \\
0.24\end{array}$ & $\begin{array}{l}0.16 \\
0.51\end{array}$ & $\begin{array}{l}0.25 \\
0.79\end{array}$ & $\begin{array}{l}0.35 \\
1.10\end{array}$ \\
\hline \multicolumn{6}{|l|}{ United States } \\
\hline $\begin{array}{l}\text { Space \& Water Heat } \\
\text { Air Conditioning \& Water Heat }\end{array}$ & $\dot{-}$ & $\begin{array}{l}1.94 \\
5.68\end{array}$ & $\begin{array}{r}4.18 \\
11.88\end{array}$ & $\begin{array}{r}6.68 \\
18.58\end{array}$ & $\begin{array}{r}9.43 \\
25.85\end{array}$ \\
\hline
\end{tabular}

* Assumes $50 \%$ diversity, i.e., two 8-hour charging periods, for space heating and air conditioning and 33\% diversity, i.e., three 4-hour charging periods, for water heating. 
EXHIBIT III- 5

Cumulative Demand By End Use:

United States

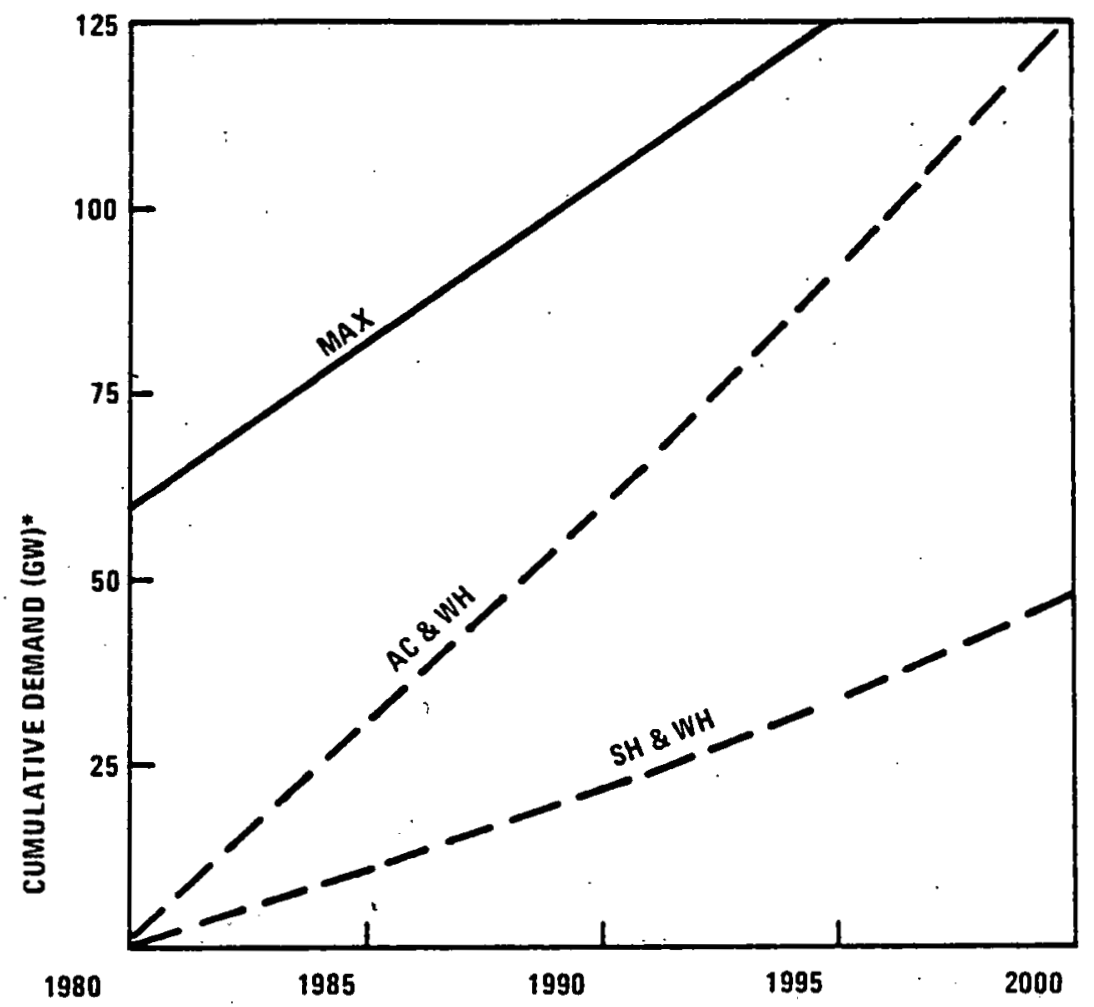

UNITED STATES

SPACE HEATING \&

WATER HEATING

AIR CONDITIONING \&

- $\quad 9.7$

20.9

33.4

47.1

WATER HEATING

28.4

59.4

92.9

130.0

* ASSUMES 50\% DIVERSITY, I.E. TWO 8-HOUR CHARgING PERIOdS FOR SPACE HEATING AND AIR CONDITIONING AND 33\% DIVERSITY, I.E. THREE 4.HOUR CHARGING PERIODS FOR WATER HEATING.

LEGEND:

MAXX: MAXIMUM TES SUPPORTABLE BY UTILITY CAPACITY

SH \& WH: SPACE HEATING AND WATER HEATING, 5\% OF NEW INSTALLATIONS

AC \& WH: AIR CONOITIOONING ANO WATER HEATING, 5\% OF NEW INSTALLATIONS 
EXHIBIT III-6

Cumulative Demand By End Use:

DOE Region
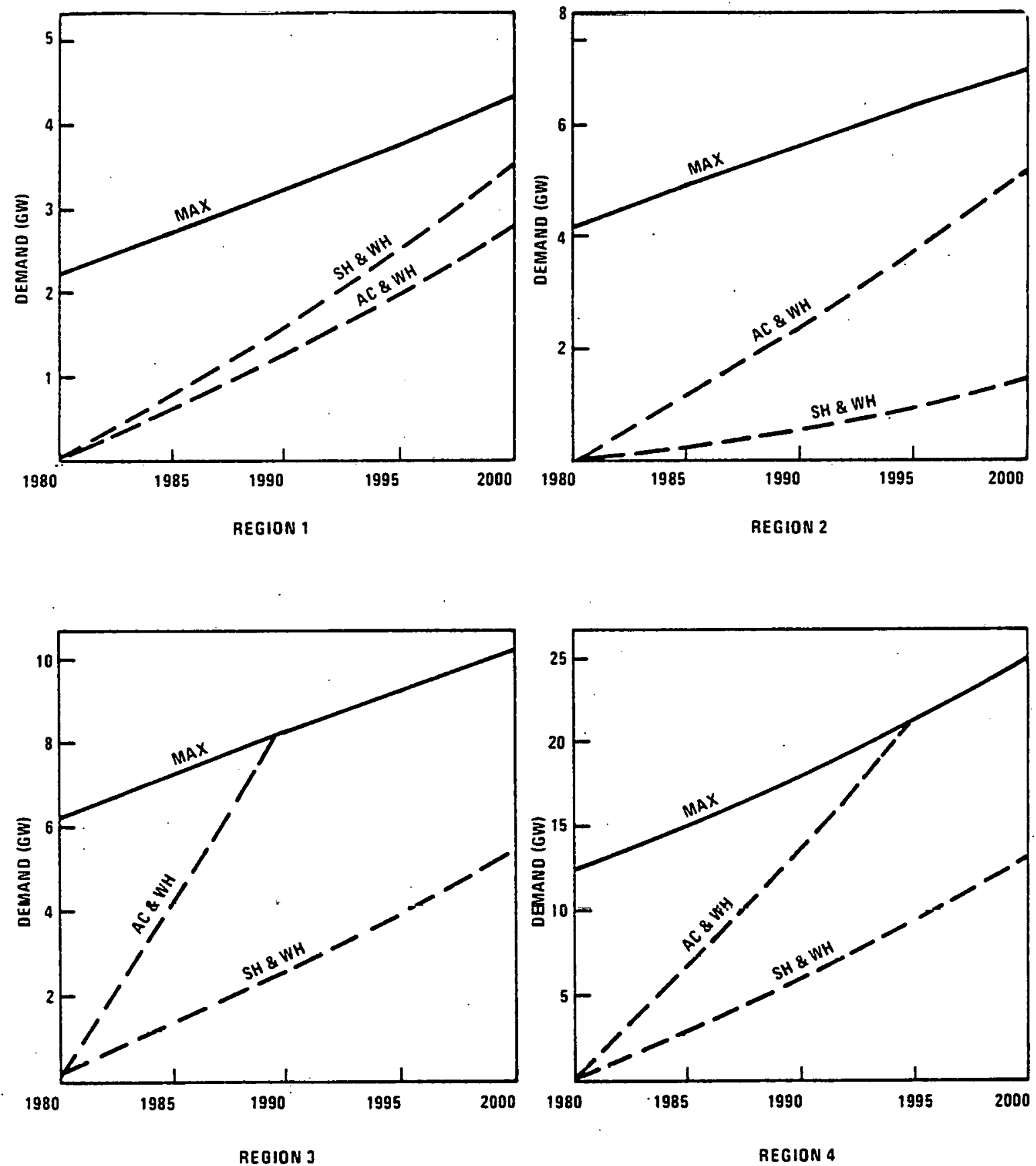

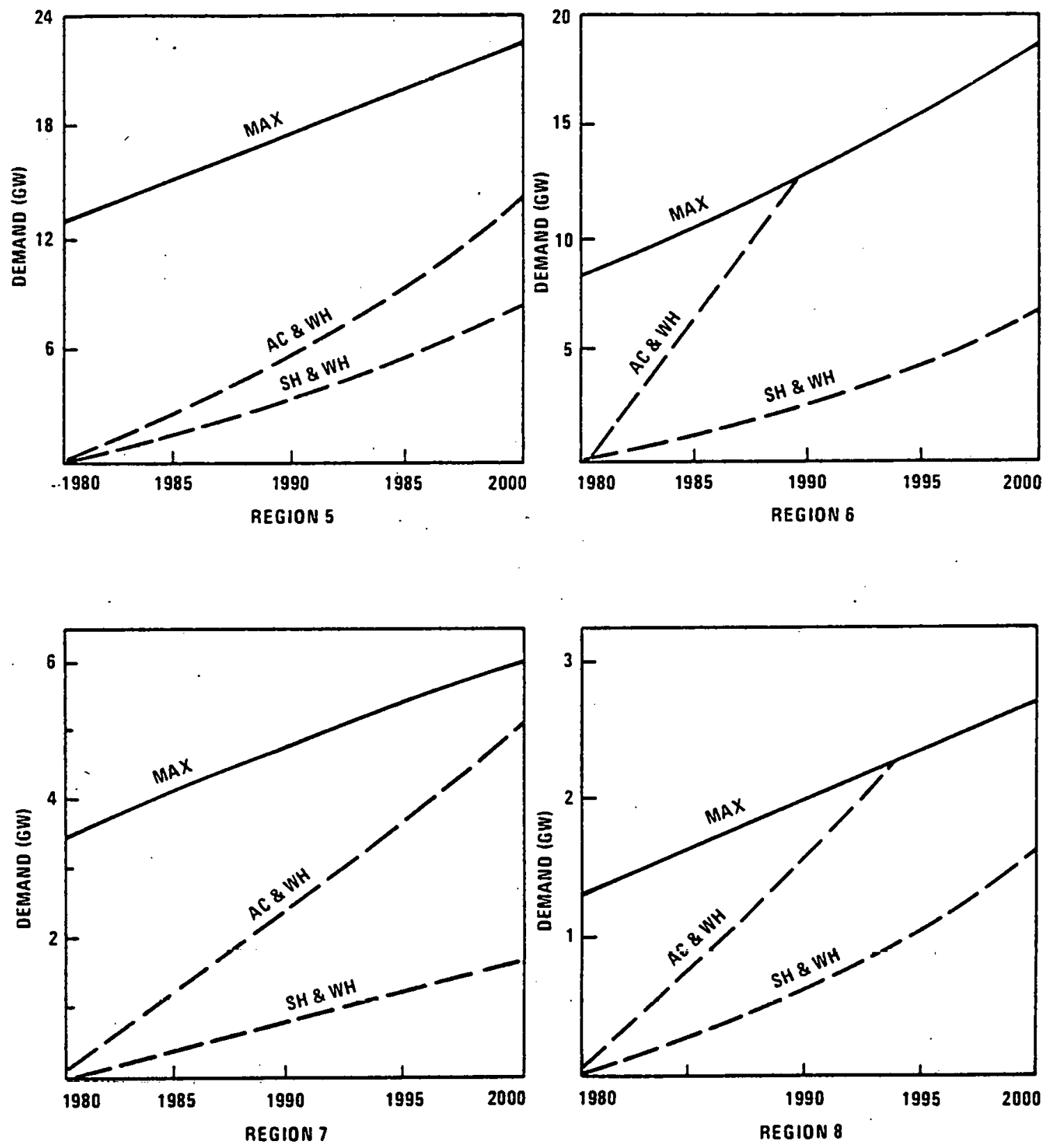

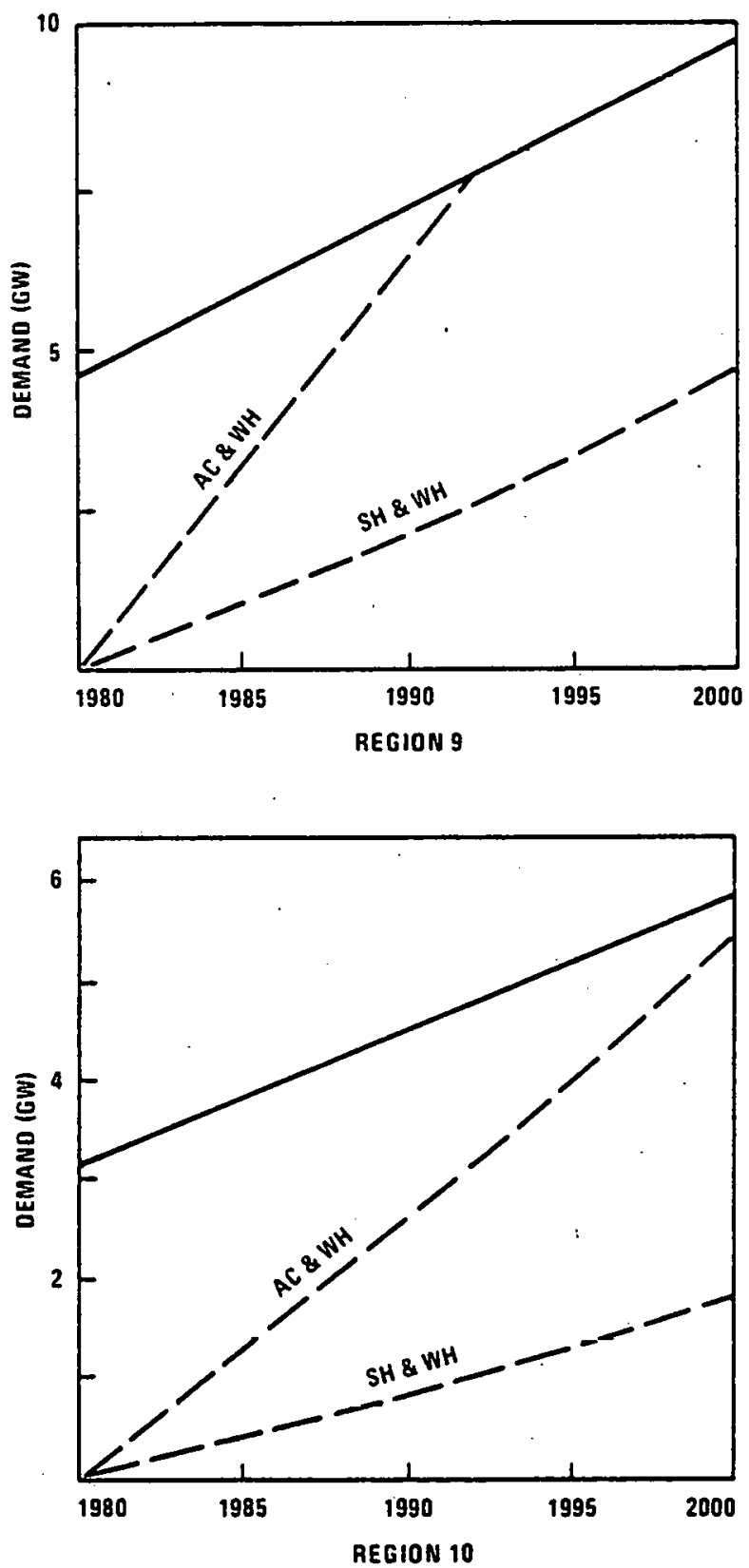

LEGEND:

MAX MAXIMUM TES SUPPORTABLE BY UTILITY CAPACITY

SH \& WH SPACE HEATING AND WATER HEATING, 5\% OF NEW INSTALLATIONS

AC \& WH AIR CONDITIONING ANO WATER HEATING, 5\% OF NEW INSTALLATIONS 
hour period, for space heating and air conditioning; and 33 percent diversity, i.e., three four-hour charging periods for water heating.

*

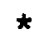

*

This chapter has concluded that a multi-gigawatt potential market exists for space heating, water heating and air conditioning in the residential and commercial sectors. This market should develop as:

- Customer awareness increases

- Utility rates favorable to TES are utilized

- Manufacturers respond to an anticipated demand for TES. 


\section{REFERENCES}

1. Asbury, J.G., and A. Kouvalis, Electric Storage Heating: The Experience in England and Wales and in the Federal Republic of Germany, Argonne National Laboratory Report ANL/ES-50, April 1976.

2. Public Service Electric and Gas Company, An Assessment of Energy Storage System Suitable for Use by Electric Utilities, Vols. I and II, EPRI EM-264 Project 225 and ERDA E(II-1)-250l, July 1976.

3. Heitner, Kenneth L., and Pamela S. Moritz, "Commercial Thermal Energy Storage for Electric Load Leveling", Public Utilities Fortnightly, May 11, 1978.

4. Swisher, James H., Chairman, Commercialization Task Force for Distributed Themal Energy Storage, Commercialization Readiness Assessment, Distributed Thermal Energy Storage, September 4, 1979.

5. Asbury, J., et.al., Assessment of Energy Storage Technologies and systems Phase I: Electric Storage Heating, Storage Air Conditioning, and Storage Hot Water Heaters, Argonne National Laboratory, Report ANL/ES-54, August 1976.

6. TRW Energy Systems Group, Consumer Energy Storage Costs for Residential Hot Water, space Heating and Space Cooling Systems, July 1976.

7. Edison Electric Institute, "Status of Rate Structure Innovations by State," July 1978 .

8. "Dodge Construction Potentials Bulletins," F.W. Dodge Division, MCGraw-Hill Information Systems Company, September 1977.

9. Residential Energy Use Simulations, 1970-2000, Version 6 (December 1978), Oak Ridge National Laboratory. Hirst, E. and J.B. Kurish, Residential Energy Use to the Year 2000: A Regional Analysis, Oak Ridge National Laboratory, ORNL/CON-17, November 1977. Hirst, E. and J. Carney, The ORNL Engineering-Economic Model of Residential Energy Uses, Oak Ridge National Laboratory, ORNL/CON-24, July 1978. 
10. Jackson, J.R. and W.S. Johnson, Commercial Energy Use: A Disaggregation by Fuel, Building Type, and End Use, Oak Ridge National Laboratory, ORNL/CON-14, February 1978.

11. The George Washington University, Factors Promoting or Inhibiting the Commercialization of Thermal Energy Storage (Draft), prepared for Argonne National Laboratory under contract 31-109-38-3648, september 30, 1977.

12. Technical Insights Inc., Heat Storage Technology.... A Key To Widespread Use of Solar Energy, Emerging Technologies No. 3, 1979.

13. Altman, M., et.al., Conservation and Better Utilization of Electric power by Means of Thermal Energy Storage and Solar Heating, Report No. NSF/RANN/SE GI27976/PR73/5 University of Pennsylvania, Philadelphia, Pennsylvania, July 1973.

14. Personal communication with Donald Denton, Jr., Vice President of Marketing, Duke Power Company Charlotte, North Carolina

15. deGrasse, R. V. "Commercialization of Electric Storage Heating," presented at the Conference on PeakLoad Pricing and Thermal Energy Storage, Chicago, Illinois, July 17, 1979.

16. RUPI, Inc. Federal Incentives for Solar Homes: An Assessment of Program Options, for the U.S. Department of Housing and Urban Development, Cambridge, Massachusetts, July 1977.

17. Nelson, Samuel H., Strategies for Commercializing Customer Thermal Energy Storage, Argonne National Laboratory, Report ANL/ES-55, December 1976.

18. Miller, Dorman, Vice President of Customer Service, American Electric Power Company, at Peak-Load Pricing and Thermal Energy Storage Conference, Chicago, Illinois, July 15-17, 1979.

19. deGrasse, Richard V., "Electric Storage Heating after Two Years", Public Utilities Fortnightly, Washington, D.C., 1977. 
20. Gahimer, John, An Overview of the DOE Thermal Storage Program, at the Peak Load Pricing and Thermal Energy storage Conference, Chicago, Illinois, July 15-17, 1979.

21. Baylin, Frank, Low Temperature Thermal Energy Storage: A State-of-the-Art Survey, Solar Energy Research Institute, SERI/RR-54-164, July 1979.

22. Booz, Allen \& Hamilton Inc, Buyer Behavior and Market Adoption of Solar Technologies, for the Office of Solar Applications, Department of Energy, August, 1979.

23. The European Experience, Peak Load Pricing and Thermal Energy Storage Conference, Chicago, Illinois, July 15-17, 1979 .

24. G. Mohr, "Electric Storage Heating," prepared for AEG-Telefunken, Frankfort, West Germany, reprinted by Control Electric Corporation, Burlington, Vermont, 1974. 


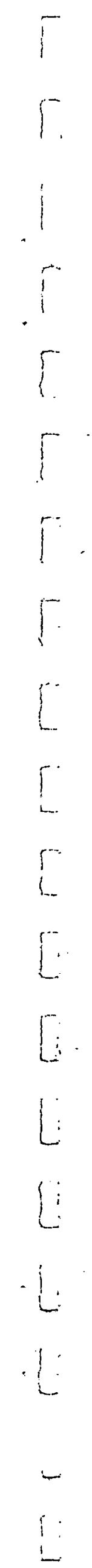

APPENDIX A

METHODOLOGY FOR THE LIFE CYCLE COST ANALYSIS 


\section{APPENDIX A}

\section{METHODOLOGY}

The methodology utilized to evaluate the economic viability of thermal energy storage systems compared the life cycle costs of TES and alternate energy technologies in different sections of the country. The comparative economics of TES with conventional space heating, water heating and air conditioning technologies was based on current prices of equipment and fuels and projections of escalating energy costs. The study did not account for:

- The uncertainty that experimental off-peak and onpeak rates would be made permanent and the sensitivity of possible reduction of rate differentials as load leveling effects are felt

Unknown effects of improvements in TES economics as the industry matures and production increases

Governmental actions and policies towards utilities and TES system, such as legislating the use of onpeak/off-peak rates by utilities, and giving demand incentives to TES consumers.

In addition, a detailed empirically-based study of buyer behavior and market adoption of TES technologies, in different parts of the country as influenced by future unknown energy environments (i.e., OPEC oil prices, the availability and price of fossil fuels, etc.) was outside the scope of this report.

Throughout the analysis, it was assumed that a rate differential was available that would be sufficiently large to offer the customer a four-year payback. This rate was optional, and applied only to the economics of TES. The following sections discuss the life cycle cost methodology. 
1. A FOUR-YEAR PAYBACK PERIOD WAS ASSUMED TO BE A PRIMARY PURCHASE REQUIREMENT FOR RESIDENTIAI AND COMMERCIAL CONSUMERS

Investors purchasing energy conservation products make their decision mainly for economic reasons. Recent studies in the solar industry, for example, have found that homeowners are influenced by potential savings on their utility bills and how soon they could recover their investment; 16 commercial customers evaluate their investment decision on the basis of payback and initial cost. 22 For both types of customers, we found that a simple payback of three to five years was often given as a necessary (but not necessarily sufficient) condition for purchase. This criteria is consistent with published information.12 For the purpose of this analysis, a payback of four years was chosen to represent a minimum investment requirement for residential and commercial purchases of TES equipment.

2. THE FOUR-YEAR PAYBACK PERIOD WAS USED TO GENERATE THE REQUIRED ELECTRIC RATE DIFFERENTIAL

The required rate differential for a four-year payback period was calculated for all residential TES applications in all ten DOE regions. It was assumed that in cases of off-peak/on-peak rates, residential customers will require half of their electrical consumption during off-peak periods and the other half during on-peak periods. The formula used to calculate the required rate difference is

$$
R R D=\left[\frac{\frac{3}{4} I C}{k W h p p}\right] \frac{I}{E}
$$

where: $\begin{aligned} \text { RRD } & =\text { Required Rate Differential } \\ \text { IC } & =\text { Incremental Cost for TES } \\ \mathrm{kWh} & =\text { Annual On-Peak Demand } \\ \mathrm{E} & =\text { TES Efficiency }\end{aligned}$

Required rate differentials for various residential and commercial TES systems have been calculated, and are shown in Exhibits $A-1$ and $A-2$. 
EXHIBIT A-l

Regional Rate Differential Requirements for a 4-year Payback Residential TES Systems

\begin{tabular}{|c|c|c|c|c|c|c|}
\hline \multirow[b]{2}{*}{ Preyinn } & :SPA:E HEATIMG TES & $\begin{array}{l}\text { DOMI:SIIC } \\
\text { HOT WATER TES }\end{array}$ & A C TFS & \multicolumn{3}{|c|}{$\begin{array}{l}\text { ENERGY REQUIREAHENTS } \\
\text { (Primary Fue 1) }\end{array}$} \\
\hline & $\begin{array}{l}\text { Central nrick TES } \\
\text { Rrol. (Systen } \\
\text { Cost } \$ 1900-\$ 3000 . \\
\text { leficiciency } 908 \text { ) }\end{array}$ & $\begin{array}{l}\text { Domestic flot } \\
\text { Water RRn (sy- } \\
\text { stem cost } \$ 200 \\
\text { Effici(ency } 1008)\end{array}$ & $\begin{array}{l}\text { ' M/C RRDI } \\
\text { (SYst.ein Cost } \\
\text { \$1S00. Effi- } \\
\text { ciency 908) }\end{array}$ & $\begin{array}{l}\text { Energy lenand } \\
\text { for Spare } \\
\text { lleatiriy in } \\
\text { muBt.u }\end{array}$ & $\begin{array}{l}\text { Energy ienand } \\
\text { for Domestic } \\
\text { llot Water in } \\
\text { mniBtu }\end{array}$ & $\begin{array}{l}\text { Einergy } \\
\text { Demand } \\
\text { For } n, c \\
\text { in nmbtu }\end{array}$ \\
\hline 1 & 4.2 & 2.0 & 92.8 & 332.65 & 51.00 & 10.03 \\
\hline 2 & 4.4 & 2,11 & 36.4 & 317.07 & 50.78 & 25.57 \\
\hline 3 & 5.8 & 2.0 & 28.7 & $236.0 \%$ & 51.18 & 32.41 \\
\hline 1 & 9.3 & 2.0 & 12.5 & $128.3 \%$ & 50.91 & 74.49 \\
\hline 5 & 4.1 & 2.0 & 35.7 & 339.44 & 51.51 & 26.09 \\
\hline 6 & 8.5 & 2.0 & 11.4 & $139.4 \vdots$ & 51.80 & 81. 74 \\
\hline 7 & 3.3 & 2.0 & 22.9 & $424.7 ?$ & 51.73 & 40.75 \\
\hline 8 & 4.0 & 2.0 & 85.7 & $353.9:$ & 51.79 & 10.86 \\
\hline y & 8.6 & 2.0 & 34.7 & $155.10^{\circ}$ & 51.80 & 26.85 \\
\hline 1.1 & 5.9 & 2.1 & 1011.8 & $245.5 \%$ & 51.75 & .92 \\
\hline
\end{tabular}

1. Repuired rote differential between off-peak and on-peak cluctric rates to constitute a 4-year ayback. 
EXHIBIT A-2

National Rate Differential Requirement for a 4-Year Payback Commercial. TES systems

\begin{tabular}{|c|c|c|c|c|c|c|}
\hline & \multicolumn{2}{|c|}{$\begin{array}{l}\text { Required Rate } \\
\text { Differential } \\
\end{array}$} & \multicolumn{2}{|c|}{$\begin{array}{cl}\text { Energy Requirement } \\
\text { mmBtu }\end{array}$} & \multicolumn{2}{|c|}{$\begin{array}{c}8 \text { Energy Use } \\
\text { On-Peak (Est.) }\end{array}$} \\
\hline $\begin{array}{l}\quad \text { Type of } \\
\text { Commercial } \\
\text { Application }\end{array}$ & $\begin{array}{c}\text { Hot Water } \\
\text { TES } \\
\text { (Incremental } \\
\text { Cost) }\end{array}$ & $\begin{array}{c}\text { Air Con } \\
\text { TES } \\
\text { (Incremental } \\
\text { Cost) }\end{array}$ & Hot Water & $A / C$ & $\begin{array}{c}\text { Hot } \\
\text { Water }\end{array}$ & $\mathrm{A} / \mathrm{C}$ \\
\hline $\begin{array}{l}\text { Office Buildings } \\
(70,000 \mathrm{sq} \cdot \mathrm{ft} .)\end{array}$ & $\begin{array}{l}0.4 \xi / \mathrm{kWh} \\
(\$ 1,700)\end{array}$ & $\begin{array}{l}1.5 \xi / \mathrm{kWh} \\
(\$ 41,600)\end{array}$ & 1,008 & 8,659 & 100 & 90 \\
\hline $\begin{array}{l}\text { Educationai Bldgs. } \\
(100,000 \mathrm{sq} . \mathrm{ft.})\end{array}$ & $\begin{array}{l}1.0 \xi / k \dot{k h h} \\
(\$ 5,500)\end{array}$ & $\begin{array}{l}1.4 \xi / \mathrm{kWh} \\
(\$ 48,000)\end{array}$ & 1,440 & 9,790 & 100 & 100 \\
\hline $\begin{array}{l}\text { Retail Buildings } \\
(40,000 \mathrm{sq} \text {. ft. })\end{array}$ & & $\begin{array}{l}1.9 \% / \mathrm{kWh} \\
(\$ 25,000\end{array}$ & & 5,540 & & 66 \\
\hline
\end{tabular}


3. THE REQUIRED RATE DIFFERENTIAL WAS USED TO GENERATE REGIONAL OFF-PEAK AND ON-PEAK ELECTRIC COST DATA

Wherever possible, the average regional electricity cost was used as the midpoint between off-peak and on-peak rates. For each region, the required rate differential was evenly distributed to generate the off-peak and on-peak costs, in the following manner:

off-peak rates $=$ current rates minus one-half $\left(-\frac{1}{2}\right)$ required rate differential

- On-peak rates $=$ current rates plus one-half $\left(+\frac{1}{2}\right)$ required rate differential

The minimum off-peak rates were allowed to be $16 / \mathrm{kWh}$ or $\$ 2.93 / \mathrm{mmBtu}$. In cases where off-peak rates would have been less than $1 \zeta / \mathrm{kW}$, the on-peak rates were adjusted to attain the required rate differential.

4. CURRENT AND PROJECTED FUEL PRICES WERE DERIVED FROM A VARIETY OF SOURCES AS DESCRIBED HEREIN

Energy prices were derived from the following sources and escalated to 1979 dollars:

Gas: AGA Gas Facts (1977)

Oil: Annual Report to Congress 1978, DOE/EIA-0173/3

Electricity: EEI Statistical Yearbook (1977)

Data Resources Inc. recently generated forecasts on conventional fuel prices for Booz, Allen \& Hamilton. Forecasts for 1980-1935 indicated the following actual fuel price escalations:

- 4.5 percent per year for oil prices

- 3.9 percent per year for residential natural gas prices

- 1.1 percent per year for residential and commercial electricity prices.

For the period between 1985 and 1990 the average fuel price escalation rates were:

2.5 percent per year for oil prices

10.2 percent per year for natural gas prices 
1.3 percent per year for residential and commercial electricity prices.

The Data Resources Inc. forecasts were developed only .1 1990. In our Life Cycle cost calculations where a .1ty-year system life was considered (1979-1999), the l price escalation rates of the 1985 to 1990 period were $\exists$ for the period between 1990 and 1999 .

THE REQUIRED RATE DIFFERENTIAL WAS KEPT INEARLY CONSTANT BY DEVELOPING INDIVIDUAL FUEL ESCALATION RATES FOR ON-PEAK AND OFF-PEAK ELECTRIC RATES

Using the same fuel price escalation rate for on-peak off-peak electric rates would result in expanding the $\supseteq$ differential between the two rates. In order to keep onstant rate differential separate escalation rates were sulated as follows:

$$
\begin{aligned}
& \mathrm{BRT}_{(1979)}-\frac{1}{2} \mathrm{RRD}=\mathrm{PO}_{(1979)} \\
& B R T \text { (1979) }^{+\frac{1}{2}} \mathrm{RRD}=P P_{\text {(1979) }}
\end{aligned}
$$

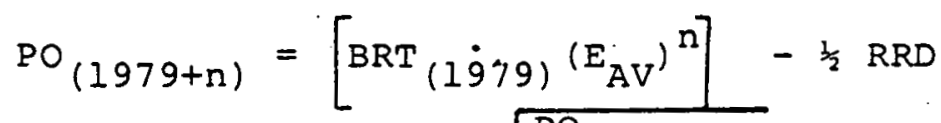

$$
\begin{aligned}
& E_{\mathrm{PO}}=\sqrt[n]{\frac{\mathrm{PO}(1979+\mathrm{n})}{\mathrm{PO}(1979)}} \\
& P P_{(1979+n)}=\left[B_{(1979)}\left(E_{A V}\right)^{n}\right]+\frac{1}{2} R R D \\
& E_{\mathrm{pP}}=\sqrt[n]{\frac{P P}{P O}(1979+n)} \\
& \begin{aligned}
B R T & =\text { Base Rate (year) } \\
R R D & =\text { Required Rate Differential } \\
B O & =\text { Off-peak Price (year) } \\
P P & =\text { On-peak Price (year) } \\
E_{A V} & =\text { Average Fuel Escalation Rate } \\
E & =\text { Fuel Escalation Rate }
\end{aligned}
\end{aligned}
$$


6. LIFE CYCLE COSTING METHODOLOGIES WERE UTILIZED TO COMPARE SYSTEM ECONOMICS

Separate life cycle methodologies were developed for residential and commercial systems. The different between the life cycle costing formulas accounts for the individual circumstances faced by each type of investor.

Residential life-cycle costing was calculated through a standard life cycle costing formula as shown in Exhibit $A-3$.

The commercial life-cycle costing methodology included the tax treatment of capital and fuel expenditures by corporations. It also accounts for capital depreciation. The formula used to calculate the life cycle cost of TES systems and competing technologies is shown in Exhibit A-4. 
EXHIBIT $A-3$

Life Cycle Costing Formula Used for Calculating Residential System Costs
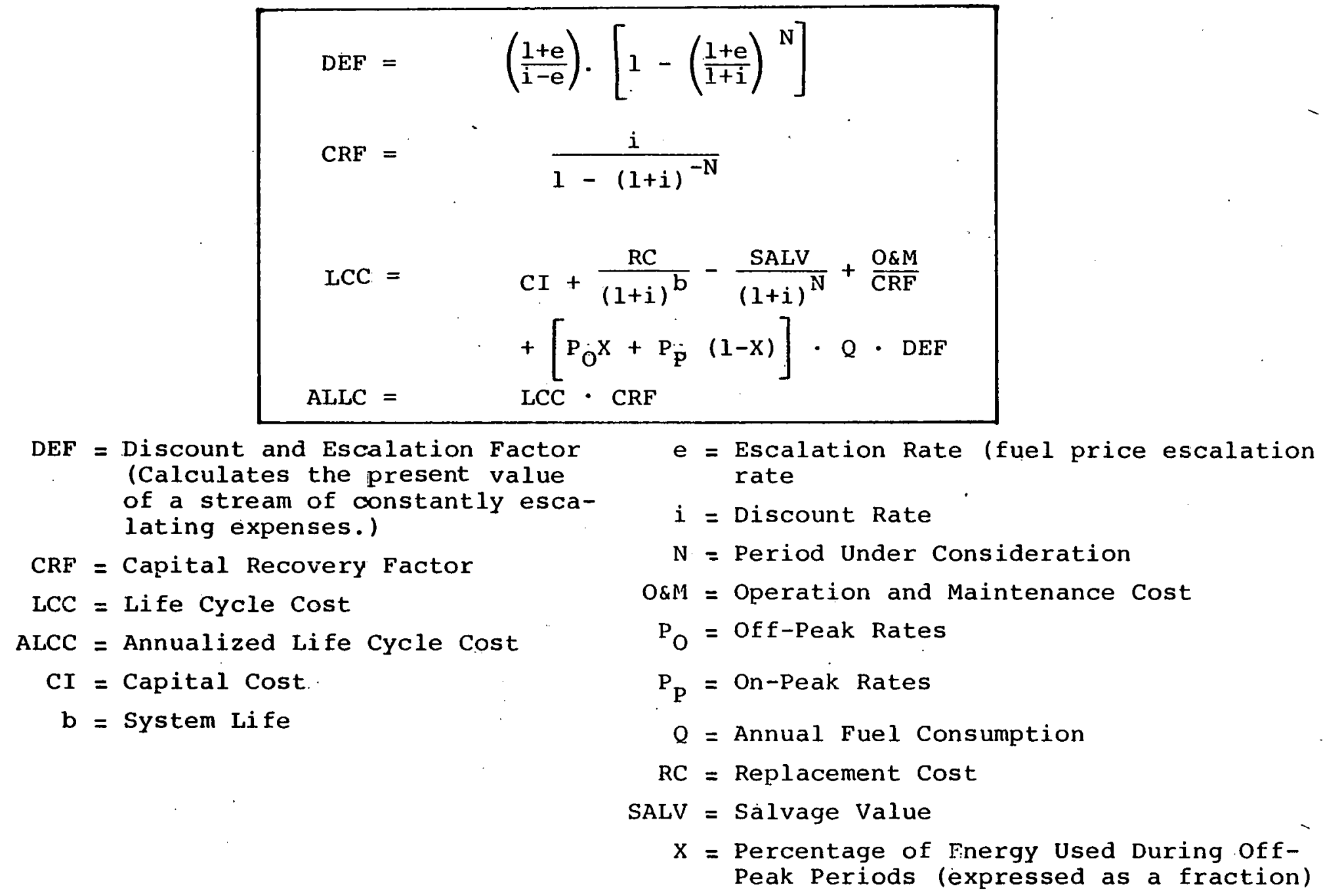

e = Escalation Rate (fuel price escalation rate

$i=$ Discount Rate

$\mathbf{N}=$ Period Under Consideration

O\&M = Operation and Maintenance Cost

$\mathrm{P}_{\mathrm{O}}=$ Off-Peak Rates

$\mathrm{P}_{\mathrm{P}}=$ On-Peak Rates

$Q=$ Annual Fuel Consumption

$\mathrm{RC}=$ Replacement Cost

SALV = Sàlvage Value

$X=$ Percentage of Fnergy Used During OffPeak Periods (expressed as a fraction) 
EXHIBIT A-4

Commercial Life Cycle Costing Formula

$\begin{aligned} & \mathrm{DEF}=\left(\frac{1+e}{i-e}\right) \cdot\left[1-\left(\frac{1+e}{1+i}\right)^{N}\right] \\ & \mathrm{CRF}= \frac{i}{1-(1+i)^{-N}} \\ &\left.\mathrm{LCC}=\mathrm{CI}+\left[\left(1-\mathrm{T}_{\mathrm{X}}\right) \cdot \frac{\mathrm{O \& M}}{\mathrm{CRF}}\right]-\frac{\mathrm{T}_{\mathrm{X} \cdot \mathrm{r} \cdot \mathrm{CI}}}{\mathrm{CRF}}-\mathrm{T}_{\mathrm{X}} \cdot \mathrm{d} \cdot \mathrm{CI}+\left(1-\mathrm{T}_{\mathrm{X}}\right) \cdot\left[\mathrm{P}_{\mathrm{O}} \mathrm{X}+\mathrm{P}_{\mathrm{P}}(1-\mathrm{X})\right] \cdot Q \cdot \mathrm{DEF}\right] \\ & \mathrm{ALCC}=\mathrm{CRF}\end{aligned}$

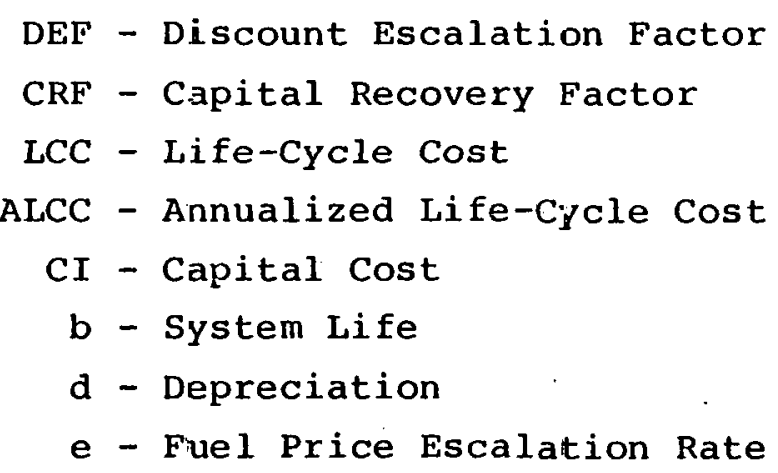

i - Discount Rate

N - Period Under Consideration

O\&M - Operation and Maintenance Cost

$P_{O}$ - Off-Peak Price of Fuel

$P_{P}$ - On-Peak Price of Fuel

Q - Annual Fuel Consumption

$r$ - Interest Rate

$T_{x}$ - Tax Rate

$X$ - Percent of Energy Used During offPeak Périods 
EXHIBIT. B-I

Common Assumptions for Residential space Heating Life Cycle cost Analysis 1

\begin{tabular}{|c|c|c|c|c|c|}
\hline \multirow[b]{2}{*}{ Parameter } & \multirow{2}{*}{$\begin{array}{c}\text { TES } \\
\text { Ceramic } \\
\end{array}$} & \multicolumn{4}{|c|}{ Conventional System } \\
\hline & & $0 i 1$ & Gas & Electric & Heat Pump \\
\hline O\&M Cost ${ }^{2}$ & $\$ 0$ & 50 & 30 & 25 & 30 \\
\hline System Life ${ }^{3}$ & 4 & & -20 & & \\
\hline Salvage Value ${ }^{4}$ & $\longleftarrow$ & 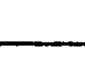 & - & . & $\longrightarrow$ \\
\hline$\%$ Off-Peak Energy 5 & 100 & NA & NA & 50 & 50 \\
\hline Fuel Escalation Rate 6 & $1.4 \%$ & 3.5 & 8.6 & $1.4 / 0.9$ & $1.4 / 0.9$ \\
\hline Discount Rate & $\leftarrow$ & & & & $\longrightarrow$ \\
\hline
\end{tabular}

1 All costs are 1979 dollars

2 Manufacturers' estimates and local HVAC service contractors' data for the average cost of a yearly service contract

3 TES system lifetime based on manufacturers' design data. Service lifetime for conventional space heating systems obtained from ASHRAE data

4 Salvage value represents the value of the ducting network

5 TES is assumed to operate totally off-peak. All other electric systems are assumed to operate 12 hours on-peak and 12 hours off-peak

6 Data Resources, Inc.' projections (November 11, 1979) for Booz, Allen \& Hamilton Inc. Escalation rates differ for on-peak and off-peak electricity and are denoted by "off-peak/on-peak." These have been adjusted to maintain a constant rate difference over the period of analysis. 
EXHIBIT B-2

Regionally Specific Assumptions

for Residential Space Heating

\begin{tabular}{cccccc}
\hline & & \multicolumn{3}{c}{ Conventional Systems } \\
\cline { 3 - 5 } DOE Region & TES & \multicolumn{3}{c}{ Electric } & Electric \\
Ceraunic Brick & $0 i l$ & Gas & Resistance & Heat Pump \\
\hline
\end{tabular}

No. 1 - New England Capital Cost $(\$)^{2} \quad 2,295$

$\begin{array}{lr}\text { Uff/Un-Peak Price (S/MBtu) } & 9.91 \\ \text { Energy (MBtu) } & 130\end{array}$

$\begin{array}{lr}2,670 & 2,444 \\ 11.90 & 7.15 \\ & 111\end{array}$

1,541

$12.84 / 19.28$

3,397

No. 2 - New York/New Jersey

Capital Cost
Off/0n-Peak Price
Energy

2,244

12.97

$2,620 \quad 2,394$

$\begin{array}{lr}2,620 & 2,394 \\ & 5.75\end{array}$

1,491

No. 3.- Mid Atlantic
Capital Cost
Off/On-Peak Price
Energy

125

106

$16.1 / 22.7$

3,239

2,192
5.60

$2,455 \quad 2,224$

$11.42 \quad 4.60$

1,321

$9.04 / 18.78$

2,867

93

79

1,196
2.93

2,066

1,729

3.80

1,050
$2.58 / 19.57$

2,077

Off/On-Peak Price Energy

$11.32 \quad 3.80$
43

$2.58 / 19.57$

$1.94 / 14.68$

No. 5 - Midwest

Capital Cost
Off/On-Peak Price
Energy

2,248

6.97

2,623

2,397

11.66

3.89

1,494

$9.75 / 16.20$

3,250

No. 6 - Southwest

Capital Cost
Off/On-Peak Price
Energy

Energy

1,911
2.93
55

$\begin{array}{lr}\text { NA } & 2,401 \\ \text { NA } & 3.60\end{array}$

.933

$3.60 / 19.13$

2,064

No. 7 - Central

Capital cost
Off/On-Peak Price
Energy

2,231
7.53
167

$2,380 \quad 2,267$

$11.50 \quad 3.25$

1,364

$9.72 / 15.0$

3,094

142

2,295
4.69
139

Capital Cost

off/On-Peak Price

Energy

$\begin{array}{rr}2,557 & 2,331 \\ 11.34 & 2.94 \\ 100 & 100\end{array}$

1,428
$5.47 / 13.6$

3,287

No. 9 - West

Capital cost
Off/On-Peak Price
Energy

2,160

2.93

NA $\quad 1,057$

3.25

1,067

$6.33 / 20.4$

50

$4.75,593$

NA $\quad 100$

2,324
2.93

Capital Cost
Off/On-Peak Price

Energy

$\begin{array}{rr}2,022 & 2,022 \\ 11.50 & 5.22 \\ 100 & 100\end{array}$

. 1.571

$1.5 / 10.3$

3,038

96

100

100

50

$1.13 / 7.73$

1. All costs are in 1979 dollars

2. Manufacturers' data

3. 1979 Edison Electric Institute Statistical Yearbook; Gas Facts; and Annual Report to Congress 1978, Volume Three, Energy Information Administration $D O E / E \overline{I A-0173 / 3}$, Adjusted for efficiency

4. Regional Energy Use Simulations, 1970-2000, Version 6 (December 1978), Oak Ridge National Laboratory

5. No oil-fired space heating units 
EXHIBIT B-3

Life Cycle Cost Analysis

for Residential space Heatingl

DOE Region
No. 1 - New England
Life Cycle Cost (LCC)(\$)
Annualized LCC $(\$)$

No. 2 - New York/New Jersey Life Cycle Cost.
Annualized LCC

No. 3 - Mid Atlantic

$$
\begin{aligned}
& \text { Life Cycle Cost } \\
& \text { Annualized LCC }
\end{aligned}
$$

No. 4 - South Atlantic

$$
\begin{aligned}
& \text { Life Cycle Cost } \\
& \text { Annualized LCC }
\end{aligned}
$$

No. 5 - Midwest

$$
\begin{aligned}
& \text { Life Cycle Cost } \\
& \text { Annualized LCC }
\end{aligned}
$$

No. 6 - Southwest

$$
\begin{aligned}
& \text { Life Cycle Cost } \\
& \text { Annualized LCC }
\end{aligned}
$$

No. 7 - Central

$$
\begin{aligned}
& \text { Life Cycle Cost } \\
& \text { Annualized LCC }
\end{aligned}
$$

No. 8 - North Central

$$
\begin{aligned}
& \text { Life Cycle Cost } \\
& \text { Annutialized LCC }
\end{aligned}
$$

No. 9 - West

$$
\begin{aligned}
& \text { Life Cycle Cost } \\
& \text { Annualized LCC }
\end{aligned}
$$

No. 10 - Nor thwest

$$
\begin{aligned}
& \text { Life Cycle. Cost } \\
& \text { Annualized LCC }
\end{aligned}
$$

$$
24,089
$$$$
1,619
$$

\section{9,785}

2,002

10,700

$$
719
$$

$$
\begin{array}{r}
21,584 \\
1,451
\end{array}
$$

4,022

270

12,517

841

\section{7,806}

1,197

30,527
2,052

4,234

285

$$
\begin{aligned}
& N A^{2} \\
& N A
\end{aligned}
$$

7,365
495

8,880

597

8,009

538

23,441

1,576

36,795

2,473

18,209

1,224

30,790

2,069

25,220

1,695

13,088

880

30,891

2,076

14,349

22,276

1,498

18,977

1,275

4,792

NA

7,680

12,569

11,278

322

NA

516

1. All costs are in 1979 dollars

2. No oil space heating systems

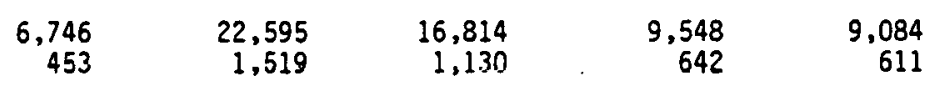


EXHIBIT B-4

Common Assumptions for Residential water Heating Life Cycle Cost Analysisl

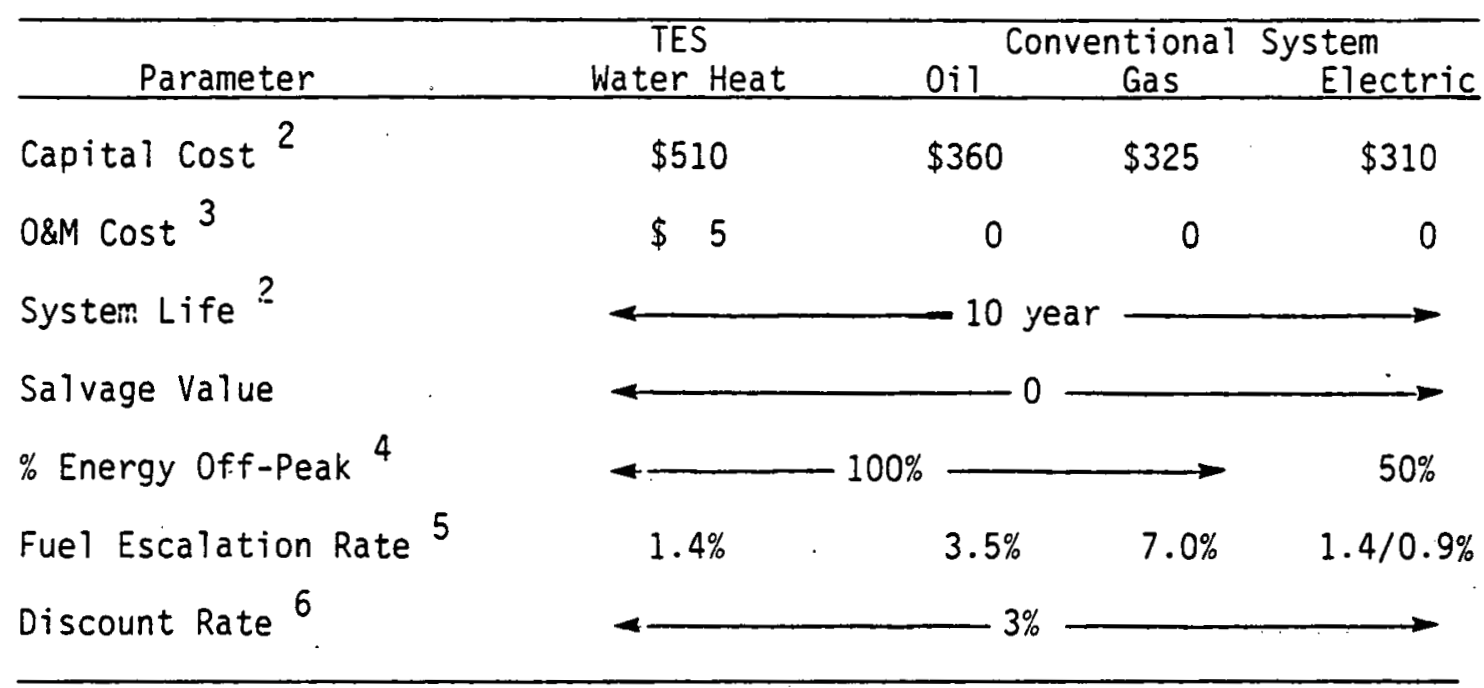

1 All costs are 1979 dollars

2 Manufacturers' data

3 Manufacturers' estimates and local HVAC service contractors' data

4 TES is assumed to operate totally off-peak. A1l other electric systems are assumed to operate 12 hours on-peak and 12 hours off-peak

5 Data Resources Inc.' projections (November 11, 1979) for Booz, Allen \& Hamilton Inc. Escalation rates differ for on-peak and off-peak electricity, and are denoted by "off-peak/on-peak." These have been adjusted to maintain a constant rate difference over the period of analysis. 
EXHIBIT $B-5$

Regionally Specific Assumptions

for Residential Water Heating

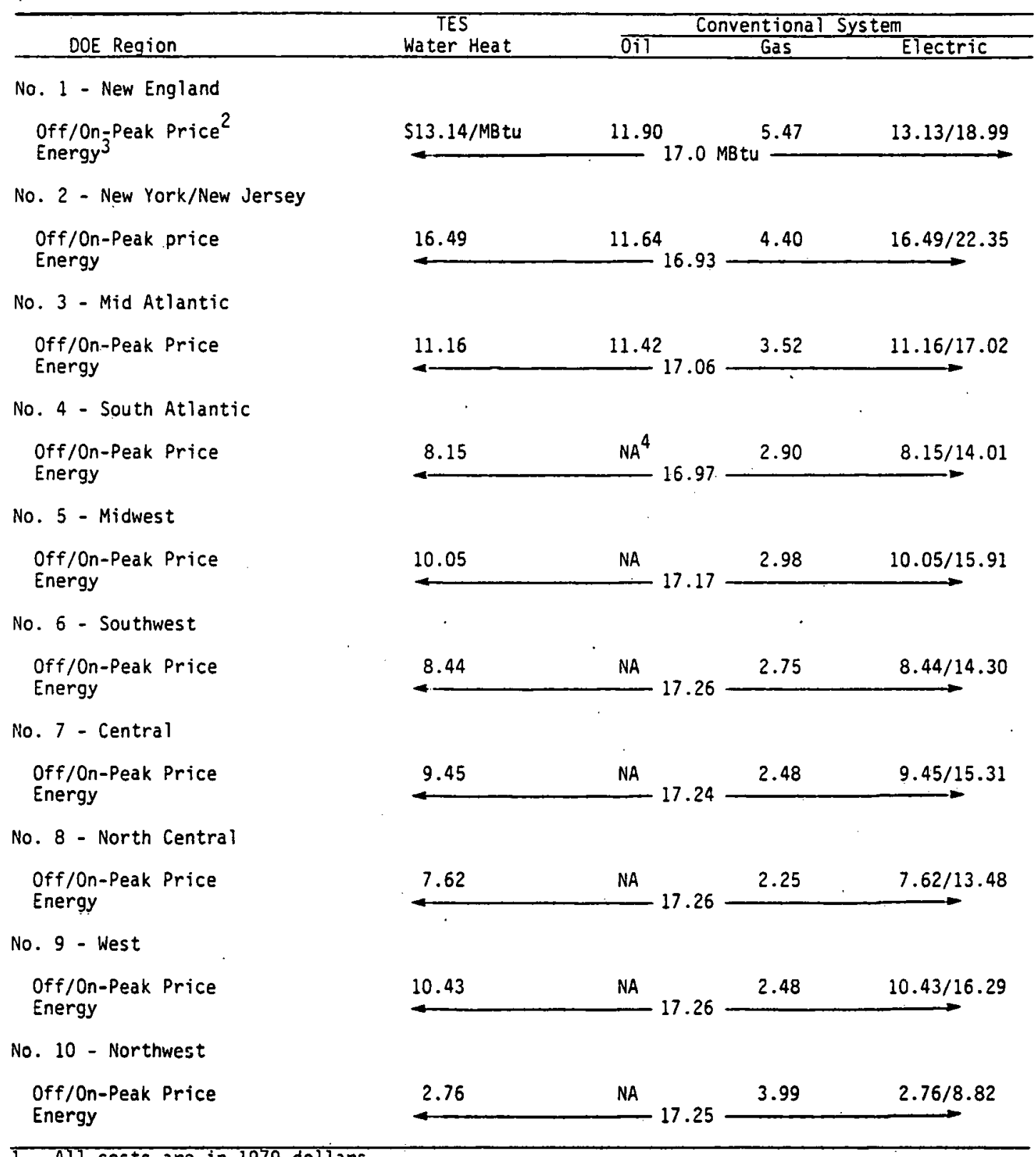

1. AlT costs are in 1979 dollars

2. 1977 Edison Electric Institute Statistical Yearbook; Gas Facts; and Annual Report to Congress 1978, Energy Information Administration, DOE/EIA-0173/3, Adjusted for efficiency

3. Regional Energy Use Simulation, 1970-2000, version 6 (December 1978), Oak Ridge National Laboratory

4. No oil-fired water heating units 
EXHIBIT B-6

Life Cycle Cost Analysis

for Residential Water Heating

\begin{tabular}{|c|c|c|c|c|}
\hline & TES & & tional & \\
\hline DOE Region & Water Heat & 01:1 & Gas & Electric \\
\hline No. 1 - New England & & & & \\
\hline $\begin{array}{l}\text { Life Cycle Cost (LCC)(\$) } \\
\text { Annualized LCC }(\$)\end{array}$ & $\begin{array}{r}2,632 \\
308\end{array}$ & $\begin{array}{r}2,427 \\
284\end{array}$ & $\begin{array}{r}1,435 \\
168\end{array}$ & $\begin{array}{r}2,825 \\
331\end{array}$ \\
\hline No. 2 - New York/New Jersey & & & & \\
\hline $\begin{array}{l}\text { Life Cycle Cost } \\
\text { Annualized LCC }(\$)\end{array}$ & $\begin{array}{r}3,154 \\
370\end{array}$ & $\begin{array}{r}2,374 \\
278\end{array}$ & $\begin{array}{r}1,215 \\
142\end{array}$ & $\begin{array}{r}3,340 \\
391\end{array}$ \\
\hline No. 3 - Mid Atlantic & & & & \\
\hline $\begin{array}{l}\text { Life Cycle Cost } \\
\text { Annualized LCC }\end{array}$ & $\begin{array}{r}2,284 \\
268\end{array}$ & $\begin{array}{r}2,351 \\
276\end{array}$ & $\begin{array}{r}1,042 \\
122\end{array}$ & $\begin{array}{r}2,524 \\
290\end{array}$ \\
\hline No. 4 - South Atlantic & & & & \\
\hline $\begin{array}{l}\text { Life Cycle Cost } \\
\text { Annualized LCC }\end{array}$ & $\begin{array}{r}1,841 \\
216\end{array}$ & $\begin{array}{l}N A^{2} \\
N A\end{array}$ & $\begin{array}{l}912 \\
107\end{array}$ & $\begin{array}{r}2,040 \\
239\end{array}$ \\
\hline No. 5 - Midwest & & & & \\
\hline $\begin{array}{l}\text { Life Cycle Cost } \\
\text { Annualized LCC }\end{array}$ & $\begin{array}{r}2,160 \\
253\end{array}$ & $\begin{array}{l}\text { NA } \\
\text { NA }\end{array}$ & $\begin{array}{l}936 \\
110\end{array}$ & $\begin{array}{r}2,362 \\
277\end{array}$ \\
\hline No. 6 - Southwest & & & & \\
\hline $\begin{array}{l}\text { Life Cycle Cost } \\
\text { Annualized LCC }\end{array}$ & $\begin{array}{r}1,910 \\
224\end{array}$ & $\begin{array}{l}\text { NA } \\
\text { NA }\end{array}$ & $\begin{array}{l}892 \\
105\end{array}$ & $\begin{array}{r}2,117 \\
248\end{array}$ \\
\hline No. 7 - Central & & & & \\
\hline $\begin{array}{l}\text { Life Cycle Cost } \\
\text { Annualized LCC }\end{array}$ & $\begin{array}{r}2,071 \\
243\end{array}$ & $\begin{array}{l}N A \\
N A\end{array}$ & $\begin{array}{r}836 \\
98\end{array}$ & $\begin{array}{r}2,275 \\
266\end{array}$ \\
\hline No. 8 - North Central & & & & \\
\hline $\begin{array}{l}\text { Life Cycle Cost } \\
\text { Annualized LCC }\end{array}$ & $\begin{array}{r}1.720 \\
202\end{array}$ & $\begin{array}{l}\text { NA } \\
\text { NA }\end{array}$ & $\begin{array}{r}789 \\
92\end{array}$ & $\begin{array}{r}1,986 \\
233\end{array}$ \\
\hline No. 9 - West & & & & \\
\hline $\begin{array}{l}\text { Life Cycle Cost } \\
\text { Annualized LCC }\end{array}$ & $\begin{array}{r}2,230 \\
261\end{array}$ & $\begin{array}{l}\text { NA } \\
\text { NA }\end{array}$ & $\begin{array}{r}836 \\
98\end{array}$ & $\begin{array}{r}2,433 \\
286\end{array}$ \\
\hline No. 10 - Northwest & & & & \\
\hline $\begin{array}{l}\text { Life Cycle cost } \\
\text { Annualized LCC }\end{array}$ & $\begin{array}{r}1,412 \\
166\end{array}$ & $\begin{array}{l}\text { NA } \\
\text { NA }\end{array}$ & $\begin{array}{r}1,147 \\
134\end{array}$ & $\begin{array}{r}1,243 \\
145\end{array}$ \\
\hline
\end{tabular}

1. A17 costs are in 1979 dollars

2. No oil water heating systems 
EXHIBIT B-7

Common Assumptions for

Residential Air Conditioning

Life Cycle Cost Analysis

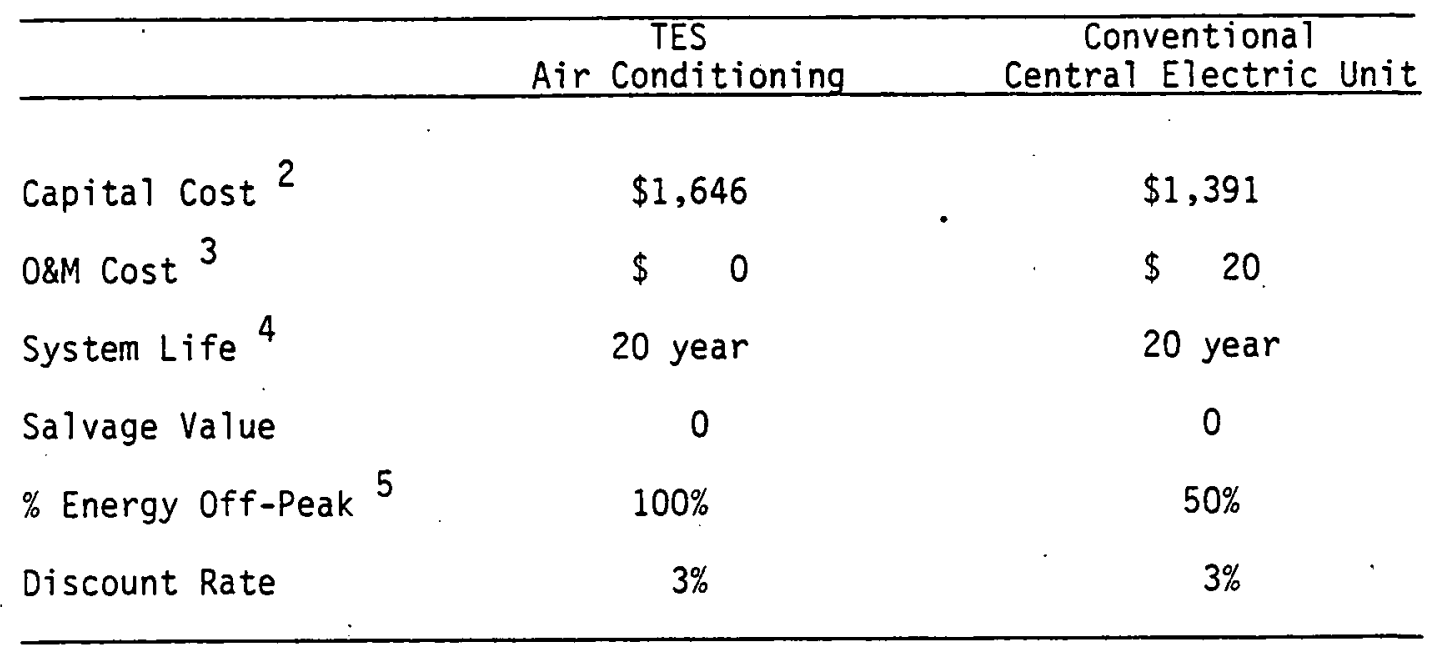

1 All costs are 1979 dollars

2 Capital cost represents the unit cost plus the cost of auxiliary equipment, such as ducting and controls

3 Manufacturers' estimates and local service contractors' data

4 Manufacturers' and ASHRAE data

5 TES is assumed to operate totally off-peak. Conventional electric air conditioning systems are assumed to operate $50 \%$ on-peak and $50 \%$ off-peak 
EXHIBIT B-8

Regionally Specific Assumptions

for Residential Air Conditioning

\begin{tabular}{llc}
\hline DOE Region/Parameter & TES & $\begin{array}{c}\text { Conventional } \\
\text { Central Electric }\end{array}$ \\
\hline
\end{tabular}

No. 3 - Mid Atlantic

$\begin{array}{lcc}\text { Off/On-Peak Price } & & \\ \text { Fuel Escalation Rate } & \$ 2.93 / \mathrm{mBtu} & 2.93 / 87.02 \\ \text { Energy }^{4} & 11.8 \% & 11.8 / 1.25 \\ & 38.8 \mathrm{mBtu} & 32.4\end{array}$

No. 4 - South Atlantic

Off/On-Peak Price

Fuel Escalation Rate

Energy

2.93

8.2

89.4

$2.93 / 39.56$

$8.2 / 1.25$

74.5

No. 6 - Southwest

Off/On-Peak Price

2.93

7.8

Fuel Escalation Rate

Energy

98.0

$2.93 / 36.33$

$7.8 / 1.25$

81.7

No. 7 - Central
off/On Peak Price
Fuel Escalation Rate Energy
2.93
10.8
49.0
$2.93 / 70.03$
$10.8 / 1.25$
40.7

1 All costs are in 1979 dollars

2. 1977 Edison Electrical Institute Statistical Yearbook

3 Data Resources, Inc. projections (November 11, 1979) for Booz, Allen \& Hamilton Inc. Escalation rates differ for on-peak and off-peak electricity and are denoted by "off-peak/on-peak." These have been adjusted to maintain a constant rate difference over the period of analysis

4. Residential Energy Use Simulations, 1970-2000, Version 6 (December 1978), Oak Ridge National Laboratory 
EXHIBIT B-9

Life Cycle Cost Analysis

for Residential Air Conditioningl

\begin{tabular}{lcc}
\hline DOE Region & TES & Conventional \\
Central Electric
\end{tabular}

No. 3 - Mid Atrantic

Life Cycle Cost (LCC)(\$)

Annualized LCC (\$)

7,173

28,071

482

1,887

No. 4 - South Atlantic

Life Cycle Cost

Annualized LCC

10,628

714

30,751

2,067

No. 6 - southwest

Life Cycle Cost Annualized LCC

11,087

745

31,142

2,093

No. 7 - Central

Life Cycle Cost Annualized LCC

7,875

28,619

529

1. Al1 costs are in 1979 dollars 
EXHIBIT $B-10$

Life Cycle Cost Analysis

for Office Buildingsl

\begin{tabular}{|c|c|c|c|c|}
\hline \multirow[b]{2}{*}{ Parameter } & \multicolumn{2}{|c|}{ Water Heating } & \multicolumn{2}{|c|}{ Air Conditioning } \\
\hline & TES & Electric & TES & Electric \\
\hline Capital Cost $(\$)^{2}$ & 2,500 & 800 & 259,100 & 217,500 \\
\hline O\&M Cost $(\$)^{2}$ & 60 & 50 & 6,000 & 5,000 \\
\hline System Life $(y r .)^{2}$ & 20 & 20 & 20 & 20 \\
\hline Tax Ràte $(\%)$ & 50 & 50 & 50 & 50 \\
\hline Interest Rate $(\%)$ & 4 & 4 & 4 & 4 \\
\hline $\begin{array}{l}\text { Off } / \text { On-Peak Price } \\
(\$ / M B t u)^{3}\end{array}$ & $11.81 /-$ & $-/ 13.0$ & $10.2 / 14.6$ & $-/ 14.6$ \\
\hline$\%$ Energy Off-Peak & 100 & 0 & 90 & 0 \\
\hline Energy Use (MBtu) ${ }^{4}$ & 1,008 & 1,008 & 9,438 & 8,659 \\
\hline $\begin{array}{l}\text { Fuel Escalation } \\
\text { Rate }(\%)^{5}\end{array}$ & 1.25 & 1.25 & $1.5 / 1.0$ & 1.0 \\
\hline Discount Rate $(\%)$ & 7 & 7 & 7 & 7 \\
\hline $\begin{array}{l}\text { Life Cycle Cost } \\
\quad \text { (LCC) }\end{array}$ & 71,575 & 77,788 & 753,998 & 856,847 \\
\hline Annualized LCC & 6,756 & 7,343 & 71,144 & 80,880 \\
\hline
\end{tabular}

1. All costs are in 1979 dotrars

2. Manufacturers' data

3. 1977 Edison Electric Institute Statistical Yearbook

4. Jackson, J. R. and W. S. Johnson, Commercial Energy Use:

A Disaggregation by Fuel, Building Type, and End Use, Oak Ridge National Laboratory, ORNL/CON-14, February 1978

5. Data Resources, Inc. projections (November 11, 1979) for Booz, Allen \& Hamilton Inc. Escalation rates for electricity differ for on-peak and off-peak electricity and are denoted by "offpeak/on-peak." These have been adjusted to maintain a constant rate difference over the period of analysis. 
EXHIBIT B-1I

Life Cycle Cost Analysis

for Educational Buildingsl

\begin{tabular}{|c|c|c|c|c|}
\hline \multirow[b]{2}{*}{ Parameter } & \multicolumn{2}{|c|}{ Water Heating } & \multicolumn{2}{|c|}{ Air Conditioning } \\
\hline & TES & Electric & TES & Electric \\
\hline Capital Cost $(\$)^{2}$ & 7,500 & 2,000 & 338,000 & 290,000 \\
\hline $0 \& M \cos t(\$)^{2}$ & 150 & 100 & 6,000 & 5,000 \\
\hline System Life $(y r .)^{2}$ & 20 & 20 & 20 & 20 \\
\hline Tax Rate (\%) & 0 & 0 & 0 & 0 \\
\hline Interest Rate (\%) & 2 & 2 & 2 & 2 \\
\hline $\begin{array}{l}\text { Off/On-Peak Price } \\
(\$ / M B t u)^{3}\end{array}$ & $10.94 /-$ & $-/ 13.87$ & $10.31 /-$ & $-/ 14.5$ \\
\hline$\%$ Energy Off-Peak & 100 & 0 & 100 & 0 \\
\hline Energy Use (MBtu) ${ }^{4}$ & 1,440 & 1,440 & 10,769 & 9,790 \\
\hline $\begin{array}{l}\text { Fuel Escalation } \\
\text { Rate }(\%)^{5}\end{array}$ & 1.3 & 1.2 & 1.5 & 1.1 \\
\hline Discount Rate & 3 & 3 & 3 & 3 \\
\hline $\begin{array}{l}\text { Life Cycle Cost } \\
\quad(L C C)\end{array}$ & 275,507 & 337,156 & $2,335,790$ & $2,712,814$ \\
\hline Annualized LCC & 18,518 & 22,662 & 157,000 & 182,344 \\
\hline
\end{tabular}

1. All costs are in 1979.dollars

2. Manufacturers' data

3. 1977 Edison Electric Institute Statistical Yearbook

4. Jackson, J. R. and W. S. Johnson, Commercial Energy Use: A Disaggregation by Fuel, Building Type, and End Use, Oak Ridge National Laboratory, ORNL/CON-14, February 1978

5. Data Resources, Inc. projections (November 11, 1979) for 800z, Allen \& Hamilton Inc. 
EXHIBIT B-12

Life Cycle Cost Analysis

for Retail Stores 1

\begin{tabular}{|c|c|c|}
\hline & & ing \\
\hline & TES & Electric \\
\hline Capital Cost $(\$)^{2}$ & 169,000 & 144,000 \\
\hline O\&M Cost $\left(\$ \$^{2}\right.$ & 6,000 & 5,000 \\
\hline System Life $(y r .)^{2}$ & 20 & 20 \\
\hline Tax RAte $(\%)$ & 50 & 50 \\
\hline Interest Rate $(\%)$ & 4 & 4 \\
\hline Off/On-Peak Price $(\$ / M B t u)^{3}$ & $9.6 / 15.2$ & $9.6 / 15.2$ \\
\hline$\%$ Energy Off-Peak & 66 & 20 \\
\hline Energy Use $(M B \text { tu })^{4}$ & 5,850 & 5,540 \\
\hline Fuel Escalation Rate $(\%)^{5}$ & $1.6 / 1.0$ & $1.6 / 1.0$ \\
\hline Discount Rate $(\%)$ & 7 & 7 \\
\hline Life Cycle Cost (LCC) & 510,043 & 546,662 \\
\hline Annualized LCC & 48,144 & 51,600 \\
\hline
\end{tabular}

1. A17 costs are in 1979 dollars

2. Manufacturers' data

3. 1977 Edison Electric Institute Statistical Yearbook

4. Jackson, J. R. and W. S. Johnson, Commercial Energy Use:

A Disaggregation by Fuel, Building Type, and End Use, Oak

Ridge Nationa 1 Laboratory, ORNL/CON-14, February 1978

5. Data Resources, Inc. projections (November 11, 1979) for Booz, Allen \& Hamilton Inc. Escalation rates for electricity differ for on-peak and off-peak electricity and are denoted by "offpeak/on-peak." These have been adjusted to maintain a constant rate difference over the period of analysis. 
APPENDIX C

PROJECTED INSTALLATIONS OF RESIDENTIAL SPACE HEATING, WATER HEATING AND AIR CONDITIONING 
EXHIBIT $\mathrm{C}-\mathrm{I}$

New Space Heat Units

Installed in Single and Multifamily Housing (Thousands of Units)

ooE Region/Fuel Type single Multi single Hulti single Multi single Multi single Multi No. 1 - New Englano

\begin{tabular}{|c|c|c|c|c|c|c|c|c|}
\hline $\begin{array}{l}\text { Electric } \\
\text { Gas } \\
\text { 0il } \\
\text { Total }\end{array}$ & $\begin{array}{r}16 \\
41 \\
73 \\
130\end{array}$ & $\begin{array}{r}29 \\
63 \\
55 \\
147\end{array}$ & $\begin{array}{r}19 \\
42 \\
83 \\
144\end{array}$ & $\begin{array}{r}33 \\
65 \\
63 \\
161\end{array}$ & $\begin{array}{r}21 \\
44 \\
86 \\
151\end{array}$ & $\begin{array}{r}34 \\
66 \\
63 \\
163\end{array}$ & 15 & $\begin{array}{r}60 \\
88 \\
23 \\
171\end{array}$ \\
\hline
\end{tabular}

No. 2 - New York/New Jersey

$\begin{array}{lrrrrrrrrrr}\text { Electric } & 10 & 25 & 12 & 27 & 13 & 27 & 18 & 36 & 26 & 52 \\ \text { Gas } & 108 & 150 & 113 & 155 & 111 & 145 & 118 & 157 & 125 & 169 \\ \text { Dil } & 64 & 155 & 68 & 167 & 70 & 160 & 54 & 132 & 35 & 94 \\ \text { Total } & 182 & 330 & 193 & 343 & 194 & 332 & 190 & 325 & 186 & 315\end{array}$

Ho. 3 - Mid Atlantic

$\begin{array}{lrrrrrrrrrrr}\text { Electric } & 66 & 51 & 75 & 55 & 82 & 56 & 96 & 62 & 117 & 71 \\ \text { Gas } & 186 & 90 & 180 & 87 & 178 & 84 & 180 & 84 & 180 & 82 \\ 0 \text { iil } & 97 & 40 & 100 & 41 & 100 & 41 & 83 & 34 & 63 & 26 \\ \text { Total } & 349 & 181 & 355 & 183 & 360 & 181 & 359 & 180 & 360 & 179\end{array}$

No. 4 - South Atlantic

Electric

Gas
OII
Tota

$\begin{array}{rrrrrrrrrr}418 & 138 & 450 & 143 & 477 & 146 & 503 & 150 & 528 & 154 \\ 166 & 51 & 147 & 50 & 153 & 53 & 160 & 57 & 167 & 60 \\ 55 & 6 & 56 & 7 & 57 & 7 & 55 & 7 & 55 & 7 \\ 639 & 195 & 653 & 200 & 687 & 206 & 718 & 214 & 771 & 221\end{array}$

No. 5 - Midwest

Electric

Gas
Oil
Total

$\begin{array}{rrrrrrrrrr}80 & 98 & 97 & 107 & 106 & 108 & 130 & 123 & 171 & 145 \\ 442 & 232 & 436 & 230 & 421 & 220 & 393 & 203 & 351 & 179 \\ 72 & 15 & 87 & 19 & 100 & 22 & 104 & 22 & 105 & 21 \\ 594 & 345 & 0<0 & 356 & 627 & 350 & 627 & 348 & 627 & 345\end{array}$

No. 6 - Southwest

Electric

Gas

Total

$\begin{array}{rrrrrrrrrr}136 & 78 & 179 & 91 & 255 & 105 & 342 & 117 & 420 & 126 \\ 265 & 37 & 226 & 28 & 163 & 17 & 97 & 10 & .9 & 47 \\ 0 & 0 & 0 & 0 & 0 & 0 & 0 & 0 & 0 & 0 \\ 401 & 115 & 405 & 119 & 418 & 122 & 439 & 127 & 467 & 132\end{array}$

No. 7 - Central

Electric

Gas
Oil
Total

$\begin{array}{rrrrrrrrrr}20 & 13 & 24 & 14 & 29 & 14 & 41 & 16 & 65 & 20 \\ 144 & 48 & 141 & 48 & 132 & 46 & 114 & 43 & 82 & 38 \\ 8 & 1 & 9 & 1 & 13 & 1 & 17 & 1 & 23 & 2 \\ 177 & 67 & 174 & 6.3 & 174 & 61 & 170 & 60 & 170 & 60\end{array}$

No. 8 - North Central

Electric

Gas
Oil
Total

$\begin{array}{rrrrrrrrrr}8 & 7 & 12 & 9 & 15 & 10 & 22 & 14 & 34 & 19 \\ 86 & 34 & 74 & 32 & 71 & 31 & 63 & 28 & 50 & 24 \\ 10 & 1 & 18 & 2 & 22 & 3 & 27 & 3 & 32 & 4 \\ 104 & 42 & 104 & 4.3 & 108 & 44 & 112 & 45 & 116 & 47\end{array}$

Ho. 9 - West

Electric

Gas
Oil
Total

$\begin{array}{rrrrrrrrrr}118 & 138 & 233 & 196 & 294 & 217 & 293 & 219 & 282 & 216 \\ 263 & 102 & 150 & 51 & 112 & 39 & 134 & 48 & 167 & 60 \\ 0 & 0 & 0 & 0 & 0 & 0 & 0 & 0 & 0 & 0 \\ 381 & 210 & 383 & 247 & 406 & 256 & 427 & 267 & 449 & 276\end{array}$

No. 10 - Northwest

\begin{tabular}{|c|c|c|c|c|c|c|c|c|c|c|}
\hline $\begin{array}{l}\text { Electric } \\
\text { Gas } \\
\text { Oil } \\
\text { Total }\end{array}$ & $\begin{array}{r}86 \\
28 \\
6 \\
120\end{array}$ & $\begin{array}{r}34 \\
9 \\
9 \\
44\end{array}$ & $\begin{array}{r}89 \\
22 \\
6 \\
117\end{array}$ & $\begin{array}{r}35 \\
8 \\
1 \\
44\end{array}$ & $\begin{array}{r}92 \\
23 \\
7 \\
-\quad 122\end{array}$ & $\begin{array}{r}35 \\
9 \\
1 \\
45\end{array}$ & $\begin{array}{r}94 \\
25 \\
6 \\
125\end{array}$ & $\begin{array}{r}35 \\
9 \\
1 \\
45\end{array}$ & $\begin{array}{r}96 \\
27 \\
4 \\
127\end{array}$ & $\begin{array}{r}35 \\
10 \\
1 \\
46\end{array}$ \\
\hline \multicolumn{11}{|c|}{ United States } \\
\hline $\begin{array}{l}\text { Electric } \\
\text { Gas } \\
\text { Di1 } \\
\text { Total }\end{array}$ & $\begin{array}{r}958 \\
1729 \\
305 \\
3072\end{array}$ & $\begin{array}{r}611 \\
816 \\
874 \\
1701\end{array}$ & $\begin{array}{r}1190 \\
1531 \\
427 \\
3148\end{array}$ & $\begin{array}{r}710 \\
754 \\
295 \\
1759\end{array}$ & $\begin{array}{r}1384 \\
1408 \\
455 \\
3356\end{array}$ & $\begin{array}{r}752 \\
710 \\
298 \\
1787\end{array}$ & $\begin{array}{r}1569 \\
1342 \\
412 \\
3323\end{array}$ & $\begin{array}{r}817 \\
717 \\
244 \\
1778\end{array}$ & $\begin{array}{r}1786 \\
1269 \\
355 \\
3410\end{array}$ & $\begin{array}{r}898 \\
716 \\
178 \\
1792\end{array}$ \\
\hline
\end{tabular}

Source: Residential Energy Use Simulations, 1970-2000, Version 6 (December 1978), Oak Ridge National Laboratory. 


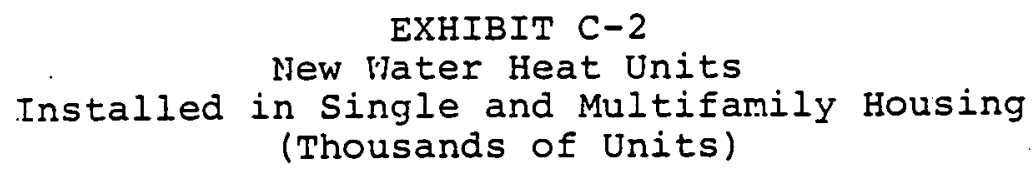

\begin{tabular}{|c|c|c|c|c|c|}
\hline OoE Region/Fuel Type & 1980 & 9985 & 1990 & 995 & 2000 \\
\hline \multicolumn{6}{|l|}{ No. 1 - New Engi and } \\
\hline $\begin{array}{l}\text { Electri= } \\
\text { Gas } \\
\text { oil } \\
\text { Total }\end{array}$ & $\begin{array}{l}191 \\
154 \\
26 \\
371\end{array}$ & $\begin{array}{r}211 \\
178 \\
30 \\
419\end{array}$ & $\begin{array}{r}222 \\
174 \\
28 \\
424\end{array}$ & $\begin{array}{r}246 \\
189 \\
99 \\
444\end{array}$ & $\begin{array}{r}261 \\
196 \\
2 \\
459\end{array}$ \\
\hline \multicolumn{6}{|l|}{ No. 2 - New York/New Jersey } \\
\hline $\begin{array}{l}\text { Electric } \\
\text { Gas } \\
0 \leqslant 1 \\
\text { Total }\end{array}$ & $\begin{array}{l}157 \\
492 \\
56 \\
705\end{array}$ & $\begin{array}{l}174 \\
507 \\
61 \\
742\end{array}$ & $\begin{array}{l}200 \\
488 \\
55 \\
743\end{array}$ & $\begin{array}{l}247 \\
474 \\
18 \\
739\end{array}$ & $\begin{array}{r}297 \\
430 \\
3 \\
730\end{array}$ \\
\hline \multicolumn{6}{|l|}{ No. 3 - Mid At lantic } \\
\hline $\begin{array}{l}\text { Electric } \\
\text { Gas } \\
\text { oil } \\
\text { Total }\end{array}$ & $\begin{array}{r}300 \\
375 \\
39 \\
714\end{array}$ & $\begin{array}{l}318 \\
374 \\
46 \\
738\end{array}$ & $\begin{array}{l}345 \\
361 \\
46 \\
752\end{array}$ & $\begin{array}{r}373 \\
355 \\
333 \\
761\end{array}$ & $\begin{array}{r}398 \\
349 \\
21 \\
768\end{array}$ \\
\hline \multicolumn{6}{|l|}{ No. 4- South Ationtic } \\
\hline $\begin{array}{l}\text { Electric } \\
\text { Gas } \\
\text { Total }\end{array}$ & $\begin{array}{r}840 \\
303 \\
1143\end{array}$ & $\begin{array}{r}875 \\
311 \\
1186\end{array}$ & $\begin{array}{r}920 \\
333 \\
1253\end{array}$ & $\begin{array}{r}960 \\
353 \\
1313\end{array}$ & $\begin{array}{r}1000 \\
374 \\
1374\end{array}$ \\
\hline \multicolumn{6}{|l|}{ No. 5 - Midwest } \\
\hline $\begin{array}{l}\text { Electric } \\
\text { Gas } \\
\text { Total }\end{array}$ & $\begin{array}{r}415 \\
792 \\
1207\end{array}$ & $\begin{array}{r}539 \\
820 \\
1359\end{array}$ & $\begin{array}{r}614 \\
769 \\
1383\end{array}$ & $\begin{array}{r}716 \\
680 \\
1396\end{array}$ & $\begin{array}{r}833 \\
572 \\
1405\end{array}$ \\
\hline \multicolumn{6}{|l|}{ No. 6 - Southwest } \\
\hline $\begin{array}{l}\text { Electric } \\
\text { Gas } \\
\text { other } \\
\text { Total }\end{array}$ & $\begin{array}{l}340 \\
348 \\
41 \\
729\end{array}$ & $\begin{array}{l}538 \\
200 \\
26 \\
764\end{array}$ & $\begin{array}{r}723 \\
66 \\
17 \\
806\end{array}$ & $\begin{array}{r}822 \\
15 \\
5 \\
842\end{array}$ & $\begin{array}{r}876 \\
2 \\
1 \\
879\end{array}$ \\
\hline \multicolumn{6}{|l|}{ No. 7 - Centrai } \\
\hline $\begin{array}{l}\text { Electric } \\
\text { Gas } \\
\text { other } \\
\text { Totol }\end{array}$ & $\begin{array}{l}103 \\
214 \\
10 \\
327\end{array}$ & $\begin{array}{r}132 \\
197 \\
6 \\
335\end{array}$ & $\begin{array}{r}182 \\
152 \\
4 \\
338\end{array}$ & $\begin{array}{r}233 \\
105 \\
2 . \\
340\end{array}$ & $\begin{array}{r}274 \\
64 \\
1 \\
339\end{array}$ \\
\hline \multicolumn{6}{|l|}{ No. 8 - North Central } \\
\hline $\begin{array}{l}\text { Electric } \\
\text { Gas } \\
\text { other } \\
\text { Tota1 }\end{array}$ & $\begin{array}{r}87 \\
112 \\
3 \\
202\end{array}$ & $\begin{array}{r}116 \\
90 \\
3 \\
209\end{array}$ & $\begin{array}{r}148 \\
70 \\
2 \\
220\end{array}$ & $\begin{array}{r}184 \\
44 \\
1 \\
229\end{array}$ & $\begin{array}{r}218 \\
20 \\
11 \\
238\end{array}$ \\
\hline \multicolumn{6}{|l|}{ No. 9 - West } \\
\hline $\begin{array}{l}\text { Electric } \\
\text { Gas } \\
\text { Other } \\
\text { Total }\end{array}$ & $\begin{array}{r}305 \\
522 \\
9 \\
936\end{array}$ & $\begin{array}{l}764 \\
104 \\
11 \\
879\end{array}$ & $\begin{array}{r}901 \\
24 \\
5 \\
930\end{array}$ & $\begin{array}{r}943 \\
31 \\
3 \\
377\end{array}$ & $\begin{array}{r}973 \\
47 \\
2 \\
1022\end{array}$ \\
\hline \multicolumn{6}{|l|}{ No. 10 - Northwest } \\
\hline $\begin{array}{l}\text { Electric } \\
\text { Gas } \\
\text { Other } \\
\text { Total }\end{array}$ & $\begin{array}{r}197 \\
24 \\
1 \\
222\end{array}$ & $\begin{array}{r}199 \\
24 \\
1 \\
224\end{array}$ & $\begin{array}{r}205 \\
27 \\
<1 \\
232\end{array}$ & $\begin{array}{r}208 \\
30 \\
<1 \\
238\end{array}$ & $\begin{array}{r}210 \\
35 \\
<1 \\
245\end{array}$ \\
\hline \multicolumn{6}{|l|}{ United Statcs } \\
\hline $\begin{array}{l}\text { Electric } \\
\text { Gas } \\
\text { oif } \\
\text { other } \\
\text { Total }\end{array}$ & $\begin{array}{r}2935 \\
3366 \\
121 \\
64 \\
6456\end{array}$ & $\begin{array}{r}3866 \\
2805 \\
137 \\
47 \\
6855\end{array}$ & $\begin{array}{r}4460 \\
2464 \\
129 \\
28 \\
7081\end{array}$ & $\begin{array}{r}4932 \\
2276 \\
60 \\
11 \\
7279\end{array}$ & $\begin{array}{r}5340 \\
2089 \\
26 \\
4 \\
7459\end{array}$ \\
\hline
\end{tabular}

Source: Kesidentida Energy Use Simulations, 19\%0-2000, Version 5 (December 1978), Oak Ridge National Laboratory. Basel ine Case for each FEA Region. 
EXHIBIT C-3

New Air Conditioning Units

Installed in Single and Multifamily Housing

(Thousands of Units)

\begin{tabular}{|c|c|c|c|c|c|c|c|c|c|c|}
\hline & \multicolumn{2}{|c|}{1980} & \multicolumn{2}{|c|}{1985} & \multicolumn{2}{|l|}{1990} & \multicolumn{2}{|c|}{1995} & \multicolumn{2}{|c|}{2000} \\
\hline & Single & Multi & Single & Multi & Single & Multi & Single & Multi & Single & Multi \\
\hline No. 1 - New England & 97 & 87 & 119 & 122 & 143 & 139 & 162 & 161 & 180 & 182 \\
\hline $\begin{aligned} \text { No. } 2 \text { - New York/ } \\
\text { New Jersey }\end{aligned}$ & 217 & 322 & 237 & 366 & 248 & 389 & 254 & 401 & 255 & 408 \\
\hline No. 3-Mid Atlantic & 385 & 198 & 420 & 214 & 448 & 225 & 465 & 232 & 478 & 237 \\
\hline No. 4 - South Atlantic & 805 & 236 & 853 & 253 & 918 & 269 & $97 ? 2$ & 285 & 1,024 & 300 \\
\hline No. 5 - Midwest & $6 \mathrm{~B} 8$ & 279 & 760 & 412 & 808 & 437 & 837 & 454 & 850 & 465 \\
\hline No. 6 - Southwest & 570 & 151 & 597 & 161 & 632 & 169 & 662 & 177 & 691 & 185 \\
\hline No. 7 - Central & 242 & 81 & 249 & 85 & 252 & 85 & 252 & 85 & 253 & 85 \\
\hline No. B - North Central & 56 & 32 & 71 & 39 & 93 & 46 & 113 & 53 & 133 & 59 \\
\hline No. 9 - West & 293 & 150 & 346 & 183 & 409 & 223 & 461 & 255 & 508 & 286 \\
\hline No. 10 - Northwest & 38 & 15 & 56 & 21 & 86 & 31 & 116 & 41 & 142 & 51 \\
\hline United States & 3,391 & 1,551 & 3,708 & 1,856 & 4,037 & 2,013 & 4,294 & 2,144 & 4,523 & 2,258 \\
\hline
\end{tabular}

Source: Residential Energy Use Simulations, 1970-2000, Version 6 (December 1978), Oak Ridge National Laboratory. 


\section{APPENDIX. D}

AVERAGE DEMAND FOR TYPICAI

RESIDENTIAL HOUSING UNITS

AND

COMMERCIAL BUILDINGS 
EXHIBIT D-1

Unit Demand Estimates

$\left(10^{3} \mathrm{Btu} / \mathrm{hr}\right)^{1}$

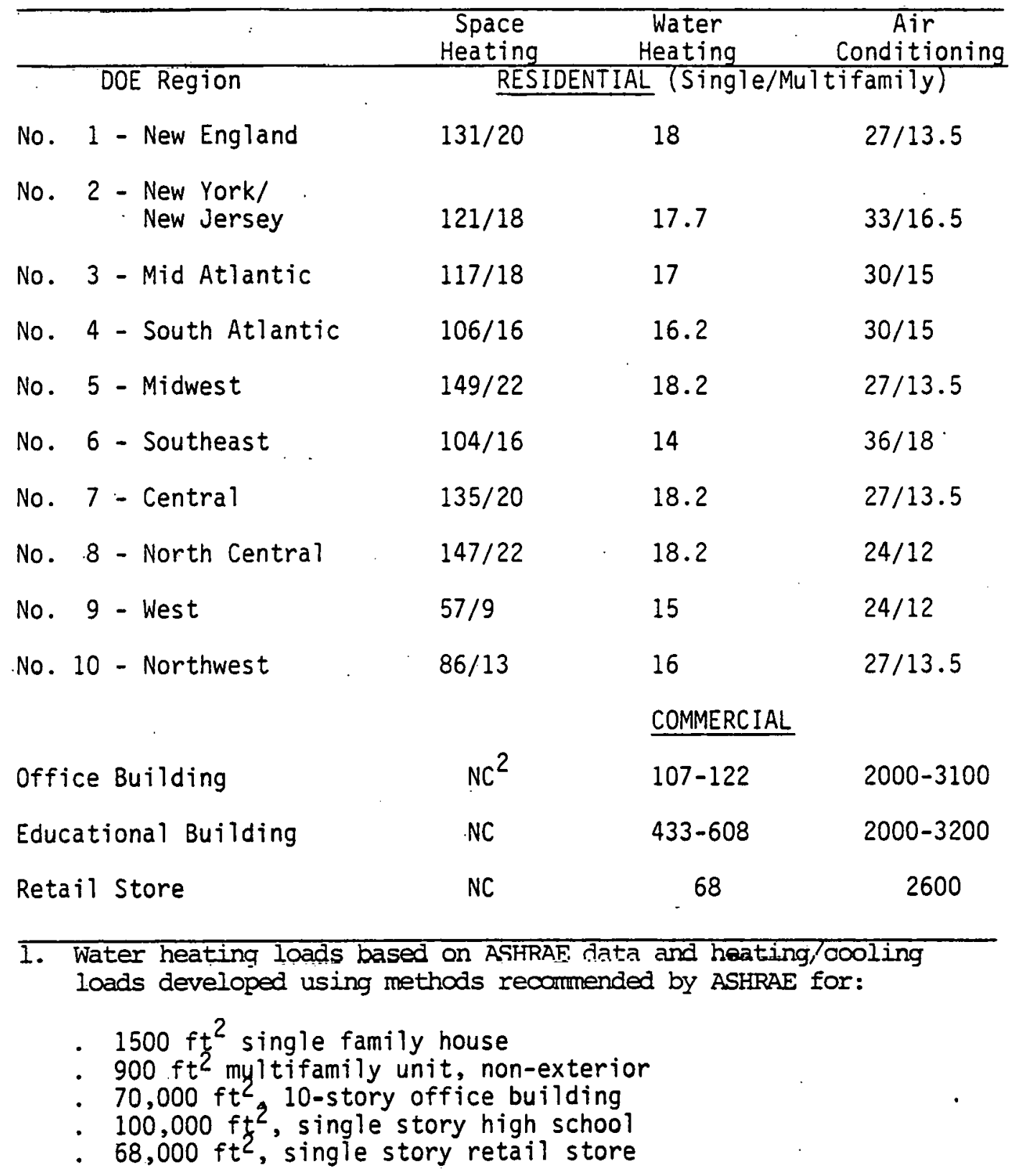

2. NC: not considered 